for

\title{
Process-Controlled Regiodivergent Copper-catalyzed Azide-alkyne Cycloadditions: Tailor-made Syntheses of 4- and 5-Bromotriazoles from Bromo(phosphoryl)ethyne
}

\author{
Yasuhiro Okuda, ${ }^{1}$ Kazuto Imafuku, ${ }^{1}$ Yoshiyuki Tsuchida, ${ }^{1}$ Tomoyo Seo, ${ }^{1}$ Haruo Akashi, ${ }^{2}$ \\ and Akihiro Orita ${ }^{1 *}$ \\ ${ }^{1}$ Department of Applied Chemistry and Biotechnology, Faculty of Engineering, ${ }^{2}$ Institute of \\ Frontier Science and Technology, Okayama University of Science, 1-1 Ridai-cho, Kita-ku, \\ Okayama 700-0005, Japan \\ Phone: +81-86-256-9533 \\ Fax: +81-86-256-4292 \\ E-mail: orita@dac.ous.ac.jp
}




\section{Table of Contents}

1. General S3

2. Detailed Reaction Procedures and Analytical Data S4-S21

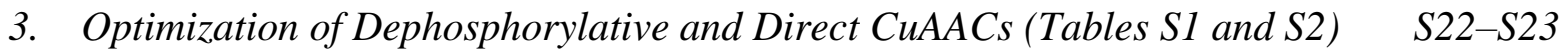

4. Control Experiment with Triisopropylsilyl (TIPS)-protected Bromoethyne 4 (Scheme S1) S24

5. Crystallographic Data (Figures $S 1$ and $S 2) \quad S 25-S 28$

6. References and Notes S29-S31

7. Copies of ${ }^{1} \mathrm{H},{ }^{13} \mathrm{C}\left\{{ }^{1} \mathrm{H}\right\},{ }^{19} \mathrm{~F}\left\{{ }^{1} \mathrm{H}\right\}$ and ${ }^{31} \mathrm{P}\left\{{ }^{1} \mathrm{H}\right\}$ NMR Charts S32-S100 


\section{General}

Unless otherwise stated, all reactions were carried out under a nitrogen atmosphere. Glassware was dried in an oven $\left(70^{\circ} \mathrm{C}\right)$ and heated under reduced pressure prior to use. Solvents were employed as eluents for all other routine operation, as well as dehydrated solvents $\left(\mathrm{CH}_{2} \mathrm{Cl}_{2}, 1,4\right.$ dioxane, THF, DMF, DMSO, $\left.\mathrm{MeOH},{ }^{n} \mathrm{PrOH}\right)$ were purchased from commercial suppliers and employed without any further purification. Toluene and $\mathrm{MeCN}$ were distilled from $\mathrm{CaH}_{2}$ prior to use. For thin layer chromatography (TLC) analyses throughout this work, Merck precoated TLC plates (silica gel $60 \mathrm{GF}_{254}, 0.25 \mathrm{~mm}$ ) were used. Silica gel column chromatography was carried out using DAISOGEL ${ }^{\mathrm{TM}}$ from Osaka Soda Co., Ltd. All NMR spectra were recorded at ambient temperature (ca. $20^{\circ} \mathrm{C}$ ) on JEOL ECS400 and JEOL ECZ400S spectrometers and referenced to the internal standard tetramethylsilane $(\delta=0.0 \mathrm{ppm})$ for ${ }^{1} \mathrm{H} \mathrm{NMR}$, deuterochloroform $(\delta=77.16$ ppm) for ${ }^{13} \mathrm{C}\left\{{ }^{1} \mathrm{H}\right\}$ NMR, ${ }^{1}$ hexafluorobenzene $(\delta=-162.2 \mathrm{ppm})$ for ${ }^{19} \mathrm{~F}\left\{{ }^{1} \mathrm{H}\right\} \mathrm{NMR}^{2}$ and triethylphosphite $(\delta=-138.60 \mathrm{ppm})$ for ${ }^{31} \mathrm{P}\left\{{ }^{1} \mathrm{H}\right\}$ NMR. ${ }^{3}$ MALDI-TOF MS was recorded on a BRUKER autoflex speed instrument. X-ray crystal analysis was conducted by X-ray diffractometer (Bruker Mac SAXS System: Cu Ka radiation), and diffraction data were collected on a Rigaku Saturn724 diffractometer using multilayer mirror monochromated Mo K $\alpha$ radiation. Data were collected and processed using CrystalClear (Rigaku). ${ }^{4}$ The structure was solved with SIR2004. ${ }^{5}$ Isotropic and full-matrix anisotropic leastsquares refinements were carried out using SHELXL97. ${ }^{6}$ The nonhydrogen atoms were refined anisotropically. Hydrogen atoms were refined using the riding model. 


\section{Detailed Reaction Procedures and Analytical Data}

Preparation of 1-Bromo(2-diphenylphosphoryl)ethyne (1): Known compound, CAS [115154649-4]. ${ }^{7}$

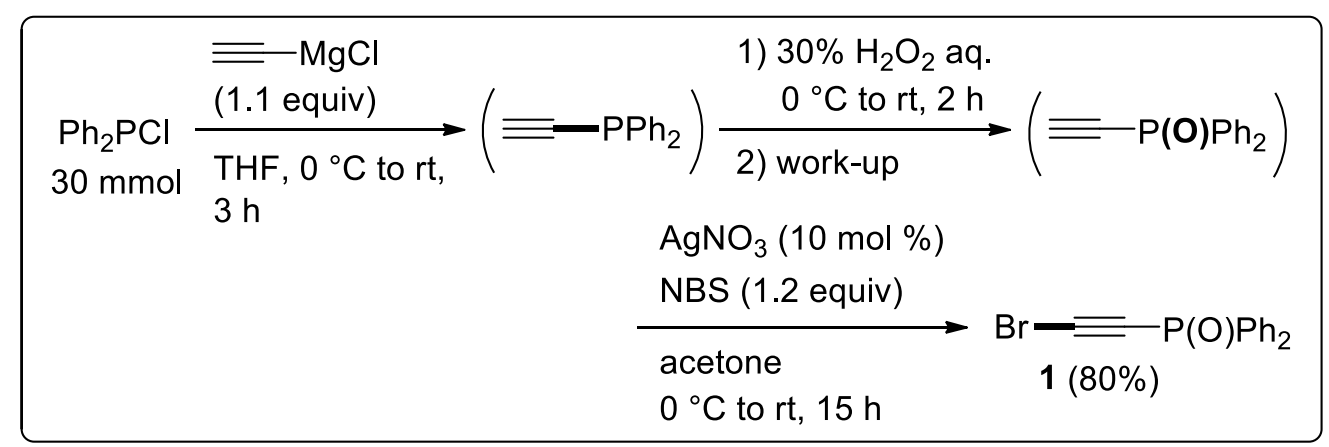

To a $300 \mathrm{~mL}$ of two-neck round-bottomed flask charged with a magnetic stirrer bar, were successively added chlorodiphenylphosphine $(5.5 \mathrm{~mL}, 30 \mathrm{mmol})$ in dry THF $(90 \mathrm{~mL})$. After the solution cooled to $0{ }^{\circ} \mathrm{C}$, ethynylmagnesium chloride $(0.5 \mathrm{M}$ in THF, $72 \mathrm{~mL}, 36 \mathrm{mmol})$ was slowly added at $0{ }^{\circ} \mathrm{C}$, and the mixture was warmed up to room temperature and stirred for $3 \mathrm{~h}$. After that, the mixture was cooled to $0{ }^{\circ} \mathrm{C}, 20 \mathrm{~mL}$ of water was added at first, and $\mathrm{H}_{2} \mathrm{O}_{2}(30 \%, 9 \mathrm{~mL})$ was subsequently added. The mixture was warmed up to room temperature and stirred for $2 \mathrm{~h}$. Saturated $5 \mathrm{~mL}$ of $\mathrm{NH}_{4} \mathrm{Cl}$ aq. was added to the reaction mixture, and organic layer was extracted with ethyl acetate and $\mathrm{H}_{2} \mathrm{O}$. The organic layer was extracted and dried over $\mathrm{MgSO}_{4}$, the solvents were removed under reduced pressure. The crude product was used for the next reaction without further purification. The crude product was diluted by $60 \mathrm{~mL}$ of acetone and cooled at $0{ }^{\circ} \mathrm{C}$. After that, silver nitrate $(510 \mathrm{mg}, 3 \mathrm{mmol})$ and $N$-bromosuccimide $(6.41 \mathrm{~g}, 36 \mathrm{mmol})$ were added at $0{ }^{\circ} \mathrm{C}$, and the mixture was warmed up to room temperature, and stirred for overnight $(\sim 15 \mathrm{~h})$. The reaction mixture was quenched with $5 \mathrm{~mL}$ of saturated $\mathrm{NH}_{4} \mathrm{Cl}$ aq., and extracted with ethyl acetate and $\mathrm{H}_{2} \mathrm{O}$. The organic layer was dried over brine and $\mathrm{MgSO}_{4}$, and the solvents were removed under reduced pressure. The crude product was subjected to column chromatography on silica gel (hexane/EtOAc, 1:1), and the volatiles were removed under vacuum for $24 \mathrm{~h}$ (*Caution: the complete removal of volatiles from the product requires so long time, otherwise a viscous liquid would be obtained) to give 1 as white solid (7.32 g, 80\% yield). ${ }^{1} \mathrm{H}$ NMR $\left(\mathrm{CDCl}_{3}, 400 \mathrm{MHz}\right): \delta$ 7.46-7.51 (m, 4H), 7.54-7.58 (m, 2H), 7.79-7.85 (m, 4H). 
General Procedure for Preparation of Azides from Corresponding Benzylic, Allylic and Alkyl Halides (Chloride or Bromide), Synthesis of 4-methoxybenzyl azide: Known compound, CAS RN [70978-37-9]. , $^{8,10,11}$

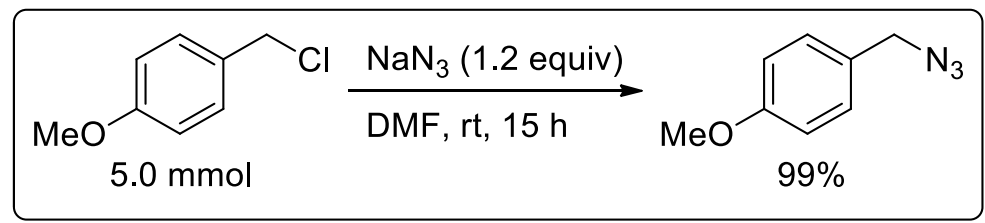

To a $100 \mathrm{~mL}$ flask charged with a magnetic stirrer bar, were added 4-chlorobenzyl chloride ( $805 \mathrm{mg}, 5.0 \mathrm{mmol}$ ), sodium azide $(390 \mathrm{mg}, 6.0 \mathrm{mmol}, 1.2$ equiv) and DMF (10 mL). The mixture was stirred at room temperature $\left(\sim 20^{\circ} \mathrm{C}\right)$ for $15 \mathrm{~h}$. After that, the reaction mixture was extracted with ethyl acetate and $\mathrm{H}_{2} \mathrm{O}$, and dried over brine and $\mathrm{MgSO}_{4}$. The crude product was purified by flash chromatography (hexane/EtOAc, 20:1) to afford the corresponding azide in 99\% yield (807 mg, colorless liquid). ${ }^{1} \mathrm{H}$ NMR ( $\mathrm{CDCl}_{3}, 400 \mathrm{MHz}$ ): $\delta 3.78$ (s, 3H), $4.23(\mathrm{~s}, 2 \mathrm{H}), 6.89(\mathrm{~d}$, $2 \mathrm{H}, J=8.8 \mathrm{~Hz}), 7.22(\mathrm{~d}, 2 \mathrm{H}, J=8.8 \mathrm{~Hz})$.

4-Trifluoromethylbenzyl azide: Known compound, CAS RN [222716-19-0]. ${ }^{8,9,12}$

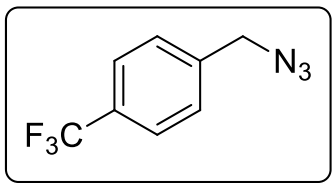

Isolated by flash chromatography (hexane/EtOAc, 20:1). Yield was $87 \%$ (876 mg, $5.0 \mathrm{mmol}$ scale, colorless liquid). ${ }^{1} \mathrm{H}$ NMR ( $\left.\mathrm{CDCl}_{3}, 400 \mathrm{MHz}\right): \delta$ $4.43(\mathrm{~s}, 2 \mathrm{H}), 7.44(\mathrm{~d}, 2 \mathrm{H}, J=8.4 \mathrm{~Hz}), 7.65(\mathrm{~d}, 2 \mathrm{H}, J=7.6 \mathrm{~Hz}) ;{ }^{19} \mathrm{~F}\left\{{ }^{1} \mathrm{H}\right\}$

$\operatorname{NMR}\left(\mathrm{CDCl}_{3}, 376 \mathrm{MHz}\right): \delta-63.1$.

4-Bromobenzyl azide: Known compound, CAS RN [107047-10-9]. ${ }^{9,10,13,14}$

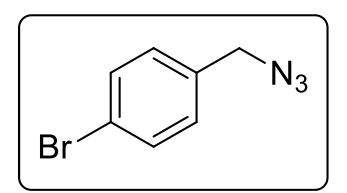

Isolated by flash chromatography (hexane/EtOAc, 20:1). Yield was $80 \%$ (848 mg, $5.0 \mathrm{mmol}$ scale, colorless liquid). ${ }^{1} \mathrm{H} \mathrm{NMR}\left(\mathrm{CDCl}_{3}, 400 \mathrm{MHz}\right)$ : $\delta 4.29(\mathrm{~s}, 2 \mathrm{H}), 7.18(\mathrm{~d}, 2 \mathrm{H}, J=8.8 \mathrm{~Hz}), 7.50(\mathrm{~d}, 2 \mathrm{H}, J=8.8 \mathrm{~Hz})$.

4-Cyanobenzyl azide: Known compound, CAS RN [84466-87-5]. ${ }^{15}$

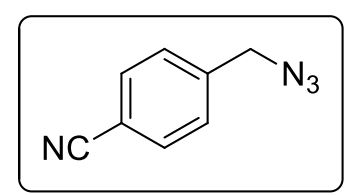
Isolated by flash chromatography (hexane/EtOAc, 10:3). Yield was 94\% (743 mg, $5.0 \mathrm{mmol}$ scale, colorless liquid). ${ }^{1} \mathrm{H} \mathrm{NMR}\left(\mathrm{CDCl}_{3}, 400 \mathrm{MHz}\right)$ : $\delta 4.45(\mathrm{~s}, 2 \mathrm{H}), 7.44(\mathrm{~d}, 2 \mathrm{H}, J=8.0 \mathrm{~Hz}), 7.69(\mathrm{~d}, 2 \mathrm{H}, J=8.0 \mathrm{~Hz})$. 
3-Methoxybenzyl azide: Known compound, CAS RN [123767-44-2]. ${ }^{12}$

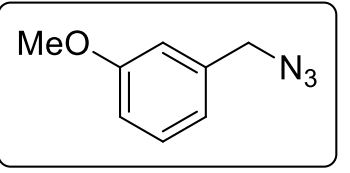

Isolated by flash chromatography (hexane/EtOAc, 20:1). Yield was 83\% (1014 mg, $7.5 \mathrm{mmol}$ scale, colorless liquid). ${ }^{1} \mathrm{H} \mathrm{NMR}\left(\mathrm{CDCl}_{3}, 400 \mathrm{MHz}\right)$ : $\delta 3.80(\mathrm{~s}, 3 \mathrm{H}), 4.28(\mathrm{~s}, 2 \mathrm{H}), 6.84-6.89(\mathrm{~m}, 3 \mathrm{H}), 7.28(\mathrm{t}, 1 \mathrm{H}, J=7.8 \mathrm{~Hz})$.

3-Bromobenzyl azide: Known compound, CAS RN [126799-86-8]. ${ }^{14,16}$

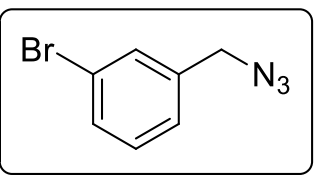

Isolated by flash chromatography (hexane/EtOAc, 20:1). Yield was 99\% (3153 mg, $15 \mathrm{mmol}$ scale, colorless liquid). ${ }^{1} \mathrm{H} \mathrm{NMR}\left(\mathrm{CDCl}_{3}, 400 \mathrm{MHz}\right)$ : $\delta 4.30(\mathrm{~s}, 2 \mathrm{H}), 7.22-7.24(\mathrm{~m}, 2 \mathrm{H}), 7.44-7.46(\mathrm{~m}, 2 \mathrm{H})$.

2-Methoxybenzyl azide: Known compound, CAS RN [300823-47-6]. ${ }^{10}$

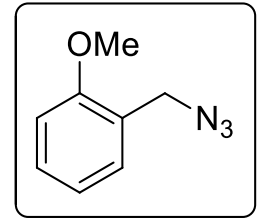

Isolated by flash chromatography (hexane/EtOAc, 20:1). Yield was 79\% (1941 $\mathrm{mg}, 15 \mathrm{mmol}$ scale, colorless liquid). ${ }^{1} \mathrm{H} \mathrm{NMR}\left(\mathrm{CDCl}_{3}, 400 \mathrm{MHz}\right): \delta 3.84$ (s, $3 \mathrm{H}), 4.34(\mathrm{~s}, 2 \mathrm{H}), 6.90(\mathrm{~d}, 1 \mathrm{H}, J=8.0 \mathrm{~Hz}), 6.92-6.96(\mathrm{~m}, 1 \mathrm{H}), 7.22-7.25(\mathrm{~m}, 1 \mathrm{H})$, $7.28-7.33(\mathrm{~m}, 1 \mathrm{H})$.

2-Bromobenzyl azide: Known compound, CAS RN [126799-87-9]. ${ }^{14}$

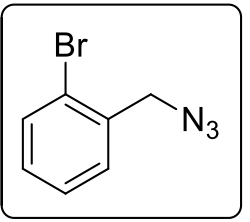

Isolated by flash chromatography (hexane/EtOAc, 20:1). Yield was 81\% (2578 mg, $15 \mathrm{mmol}$ scale, colorless liquid). ${ }^{1} \mathrm{H} \mathrm{NMR}\left(\mathrm{CDCl}_{3}, 400 \mathrm{MHz}\right): \delta 4.46$ (s, $2 \mathrm{H}), 7.17(\mathrm{td}, 1 \mathrm{H}, J=7.8,1.7 \mathrm{~Hz}), 7.31(\mathrm{td}, 1 \mathrm{H}, J=7.6,1.2 \mathrm{~Hz}), 7.37(\mathrm{dd}, 1 \mathrm{H}, J=$ 7.6, 1.6 Hz), $7.58(\mathrm{dd}, 1 \mathrm{H}, J=8.0,1.2 \mathrm{~Hz})$.

2-(Azidemethyl)naphthalene: Known compound, CAS RN [164269-42-5]. ${ }^{10,11}$

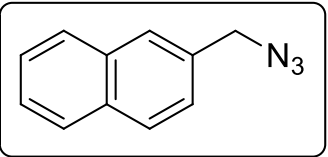

Isolated by flash chromatography (hexane/EtOAc, 10:1). Yield was 99\% (3153 mg, $15 \mathrm{mmol}$ scale, off-white powder). ${ }^{1} \mathrm{H} \mathrm{NMR}\left(\mathrm{CDCl}_{3}, 400 \mathrm{MHz}\right.$, $\mathrm{rt}): \delta 4.48(\mathrm{~s}, 2 \mathrm{H}), 7.41(\mathrm{dd}, J=8.6,1.4 \mathrm{~Hz}, 1 \mathrm{H}), 7.48-7.51(\mathrm{~m}, 2 \mathrm{H}), 7.76(\mathrm{~s}$, 1H), 7.82-7.87 (m, 3H).

(2-Azidoethyl)benzene: Known compound, CAS RN [6926-44-9]. ${ }^{11}$

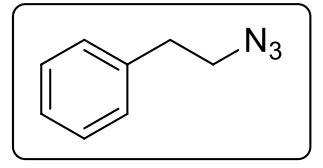

Isolated by flash chromatography (hexane/EtOAc, 20:1). Yield was 83\% (1223 mg, $10 \mathrm{mmol}$ scale, colorless liquid). ${ }^{1} \mathrm{H} \mathrm{NMR}\left(\mathrm{CDCl}_{3}, 400 \mathrm{MHz}\right): \delta$ $2.87(\mathrm{t}, 2 \mathrm{H}, J=7.2 \mathrm{~Hz}), 3.48(\mathrm{t}, 2 \mathrm{H}, J=7.2 \mathrm{~Hz}), 7.19-7.25(\mathrm{~m}, 3 \mathrm{H}), 7.29-7.33$

$(\mathrm{m}, 2 \mathrm{H})$. 
(3-Azidoprop-1-en-1-yl)benzene: Known compound, CAS RN [57294-86-7]. ${ }^{15}$

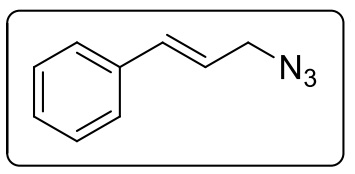

Isolated by flash chromatography (hexane/EtOAc, 20:1). Yield was 87\% (1388 mg, $10 \mathrm{mmol}$ scale, pale yellow liquid). ${ }^{1} \mathrm{H} \mathrm{NMR}\left(\mathrm{CDCl}_{3}, 400\right.$ MHz): $\delta 3.90(\mathrm{~d}, 2 \mathrm{H}, J=6.4 \mathrm{~Hz}), 6.20(\mathrm{dt}, 1 \mathrm{H}, J=16.0,6.6 \mathrm{~Hz}), 6.62(\mathrm{~d}$, $1 \mathrm{H}, J=15.6 \mathrm{~Hz}), 7.23-7.27(\mathrm{~m}, 1 \mathrm{H}), 7.31(\mathrm{t}, 2 \mathrm{H}, J=8.2 \mathrm{~Hz}), 7.38(\mathrm{~d}, 2 \mathrm{H}, J=8.4 \mathrm{~Hz})$.

Azidocyclohexane: Known compound, CAS RN [19573-22-9]. ${ }^{11}$

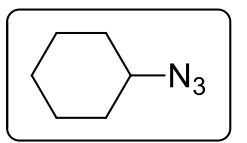

Isolated by flash chromatography (hexane). Yield was $71 \%(441 \mathrm{mg}, 5.0 \mathrm{mmol}$ scale, reaction at $80{ }^{\circ} \mathrm{C}$, colorless liquid). ${ }^{1} \mathrm{H} \mathrm{NMR}\left(\mathrm{CDCl}_{3}, 400 \mathrm{MHz}\right): \delta 1.19$ $1.42(\mathrm{~m}, 6 \mathrm{H}), 1.55-1.60(\mathrm{~m}, 1 \mathrm{H}), 1.74-1.78(\mathrm{~m}, 2 \mathrm{H}), 1.88-1.92(\mathrm{~m}, 2 \mathrm{H}), 3.30$

$3.36(\mathrm{~m}, 1 \mathrm{H})$.

General Procedure for Preparation of Aryl Azides from Corresponding Anilines, 1-Azido-4-chlorobenzene: Known compound, CAS RN [3296-05-7]. ${ }^{17}$

(

To a $100 \mathrm{~mL}$ flask charged with a magnetic stirrer bar, were added 4-chloroaniline (383 $\mathrm{mg}, 3.0 \mathrm{mmol})$ and ethyl acetate $(6 \mathrm{~mL})$. The solution was cooled at $0{ }^{\circ} \mathrm{C}$, and concentrated $\mathrm{HCl}$ solution $(1.2 \mathrm{~mL})$ was added. After short minutes, sodium nitrite (497 mg, $3.6 \mathrm{mmol})$ in $\mathrm{H}_{2} \mathrm{O}(8$ $\mathrm{mL}$ ) was slowly added, and stirred for $1 \mathrm{~h}$. Sodium azide (468 mg, $3.6 \mathrm{mmol}$ ) in $\mathrm{H}_{2} \mathrm{O}(8 \mathrm{~mL})$ was added to the reaction mixture in dropwise. After that, the mixture was warmed up to room temperature and stirred for $3 \mathrm{~h}$. The resulted mixture was extracted with ethyl acetate and $\mathrm{H}_{2} \mathrm{O}$, and dried over brine and $\mathrm{MgSO}_{4}$. The crude product was purified by flash chromatography (hexane/EtOAc, 20:1) to afford the corresponding azide in $73 \%$ yield (336 mg, brown liquid). ${ }^{1} \mathrm{H}$ $\mathrm{NMR}\left(\mathrm{CDCl}_{3}, 400 \mathrm{MHz}\right): \delta 6.96(\mathrm{~d}, 2 \mathrm{H}, J=8.8 \mathrm{~Hz}), 7.31(\mathrm{~d}, 2 \mathrm{H}, J=8.8 \mathrm{~Hz})$.

1-Azido-4-bromobenzene: Known compound, CAS RN [2101-88-4]. ${ }^{18}$

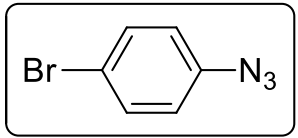

Isolated by flash chromatography (hexane/EtOAc, 20:1). Yield was 87\% (858 mg, $5.0 \mathrm{mmol}$ scale, brown liquid). ${ }^{1} \mathrm{H} \mathrm{NMR}\left(\mathrm{CDCl}_{3}, 400 \mathrm{MHz}\right): \delta$ $6.87(\mathrm{~d}, 2 \mathrm{H}, J=7.6 \mathrm{~Hz}), 7.43(\mathrm{~d}, 2 \mathrm{H}, J=8.0 \mathrm{~Hz})$. 
Representative Procedure for One-shot Dephosphorylative CuAAC of Bromo(phosphoryl)ethyne 1, Synthesis of 1-benzyl-4-bromo-1,2,3-triazole (2a): Known compound, CAS RN [31875-99-7]. ${ }^{19,20}$

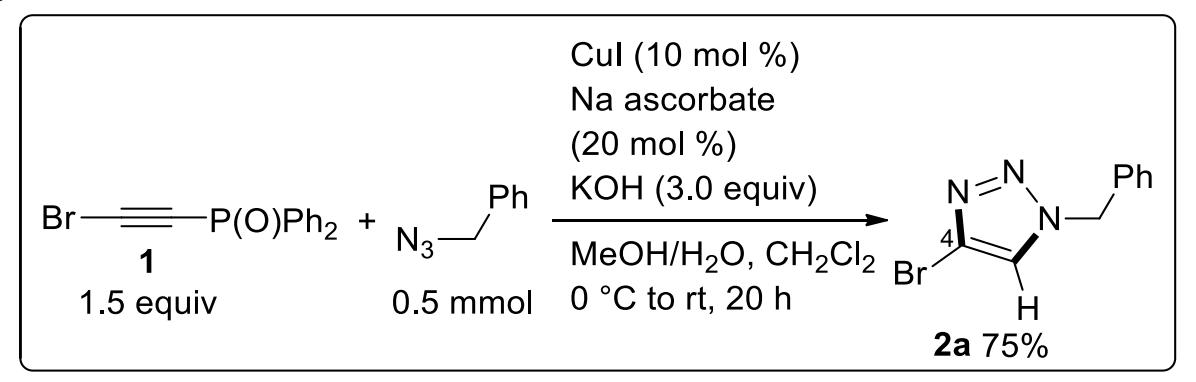

To a $50 \mathrm{~mL}$ of two-neck round-bottomed flask charged with a magnetic stirrer bar, were added bromo(phosphoryl)ethyne 1 ( $229 \mathrm{mg}, 0.75 \mathrm{mmol}$ ), copper iodide $(9.5 \mathrm{mg}, 0.05 \mathrm{mmol}$ ), sodium ascorbate $(19.8 \mathrm{mg}, 0.10 \mathrm{mmol}), \mathrm{KOH}(84 \mathrm{mg}, 1.5 \mathrm{mmol})$ and $\mathrm{CH}_{2} \mathrm{Cl}_{2}(5 \mathrm{~mL})$. The mixture was stirred at $0{ }^{\circ} \mathrm{C}$ for $5 \mathrm{~min}$, then benzyl azide $(67 \mathrm{mg}, 0.50 \mathrm{mmol}$ ) was added. After that, the mixture was warmed up to room temperature and stirred for $20 \mathrm{~h}$. The resulted mixture was extracted with ethyl acetate and $\mathrm{H}_{2} \mathrm{O}$, and dried over brine and $\mathrm{MgSO}_{4}$. The crude product was purified by flash chromatography (hexane/EtOAc, 5:1 2:1) to afford the corresponding 4-bromotriazole 2a in 75\% yield (89 mg, white solid). mp: $72-73{ }^{\circ} \mathrm{C} .{ }^{1} \mathrm{H}$ NMR $\left(\mathrm{CDCl}_{3}, 400 \mathrm{MHz}\right): \delta 5.53$ (s, 2H), 7.26-7.29 $(\mathrm{m}, 2 \mathrm{H}), 7.38-7.40(\mathrm{~m}, 3 \mathrm{H}), 7.45(\mathrm{~s}, 1 \mathrm{H}) .{ }^{13} \mathrm{C}\left\{{ }^{1} \mathrm{H}\right\} \mathrm{NMR}\left(\mathrm{CDCl}_{3}, 101 \mathrm{MHz}\right): \delta$ 55.0, 121.0, 123.7, 128.3, 129.2, 129.4, 133.9. HRMS (MALDI-TOF) m/z: $[\mathrm{M}+\mathrm{H}]^{+}$Calcd for $\mathrm{C}_{9} \mathrm{H}_{9} \mathrm{BrN}_{3} 237.9980$; Found 237.9964. The regioselectivity of $\mathbf{2 a}$ was determined by the single-crystal X-ray analysis (Figure S1). We assumed that the other derivatives $\mathbf{2 b}-\mathbf{h}$ and $\mathbf{2} \mathbf{j}$ would exhibit the same regioselectivity.

\footnotetext{
4-Bromo-1-(p-methoxybenzyl)-1,2,3-triazole $\quad(2 b): \quad$ Known compound, CAS RN [1907649-08-4]. ${ }^{20}$

Isolated by flash chromatography (hexane/EtOAc, 2:1). Yield was 65\% (87 mg, white solid). mp: 99-100 ${ }^{\circ} \mathrm{C} .{ }^{1} \mathrm{H}$ NMR $\left(\mathrm{CDCl}_{3}, 400\right.$ MHz): $\delta 3.82(\mathrm{~s}, 3 \mathrm{H}), 5.46(\mathrm{~s}, 2 \mathrm{H}), 6.91(\mathrm{~d}, 2 \mathrm{H}, J=8.8 \mathrm{~Hz}), 7.24(\mathrm{~d}$, $2 \mathrm{H}, J=8.8 \mathrm{~Hz}), 7.41(\mathrm{~s}, 1 \mathrm{H}) .{ }^{13} \mathrm{C}\left\{{ }^{1} \mathrm{H}\right\} \mathrm{NMR}\left(\mathrm{CDCl}_{3}, 101 \mathrm{MHz}\right): \delta$

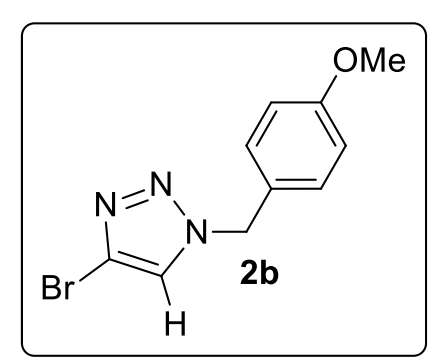
54.6, 55.5, 114.8, 120.9, 123.5, 125.9, 130.0, 160.4. HRMS (MALDI-TOF) m/z: $[\mathrm{M}+\mathrm{Na}]^{+} \mathrm{Calcd}$ for $\mathrm{C}_{10} \mathrm{H}_{10} \mathrm{BrN}_{3} \mathrm{NaO} 289.9905$; Found 289.9938 .
} 


\section{4-Bromo-1-(p-trifluoromethylbenzyl)-1,2,3-triazole (2c): New}

compound.

Isolated by flash chromatography (hexane/EtOAc, 2:1). Yield was 89\% (136 mg, white solid). mp: $105-106{ }^{\circ} \mathrm{C} .{ }^{1} \mathrm{H}$ NMR $\left(\mathrm{CDCl}_{3}, 400\right.$ $\mathrm{MHz}): \delta 5.60(\mathrm{~s}, 2 \mathrm{H}), 7.39(\mathrm{~d}, 2 \mathrm{H}, J=8.0 \mathrm{~Hz}), 7.50(\mathrm{~s}, 1 \mathrm{H}), 7.66(\mathrm{~d}$, $2 \mathrm{H}, J=8.0 \mathrm{~Hz}) .{ }^{13} \mathrm{C}\left\{{ }^{1} \mathrm{H}\right\} \mathrm{NMR}\left(\mathrm{CDCl}_{3}, 101 \mathrm{MHz}\right): \delta 54.2,121.4$,

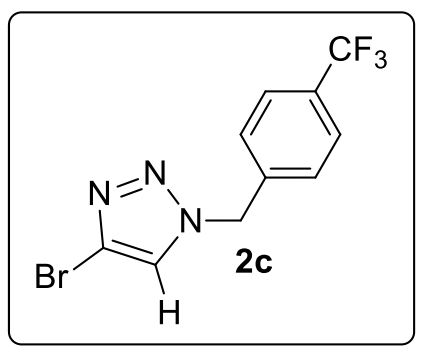
$123.8(\mathrm{q}, J=272 \mathrm{~Hz}), 123.9,126.4(\mathrm{q}, J=3.9 \mathrm{~Hz}), 128.5,131.5(\mathrm{q}, J=33 \mathrm{~Hz}), 137.9 .{ }^{19} \mathrm{~F}\left\{{ }^{1} \mathrm{H}\right\}$ NMR ( $\left.\mathrm{CDCl}_{3}, 376 \mathrm{MHz}\right): \delta-63.3$. HRMS (MALDI-TOF) m/z: $[\mathrm{M}+\mathrm{H}]^{+}$Calcd for $\mathrm{C}_{10} \mathrm{H}_{8} \mathrm{BrF}_{3} \mathrm{~N}_{3}$ 305.9854; Found 305.9874.

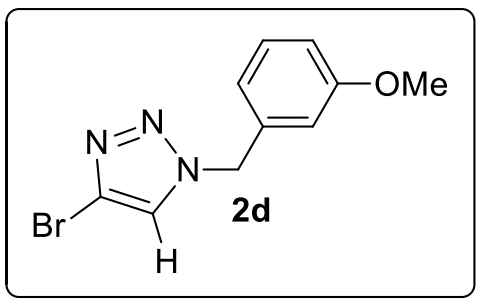

4-Bromo-1-(m-methoxybenzyl)-1,2,3-triazole (2d): Known compound, CAS RN [1907649-11-9].

Isolated by flash chromatography (hexane/EtOAc, 2:1). Yield was $88 \%$ (118 mg, white solid). mp: $87-88{ }^{\circ} \mathrm{C} .{ }^{1} \mathrm{H}$ NMR $\left(\mathrm{CDCl}_{3}, 400\right.$ $\mathrm{MHz}): \delta 3.80(\mathrm{~s}, 3 \mathrm{H}), 5.49(\mathrm{~s}, 2 \mathrm{H}), 6.80(\mathrm{t}, 1 \mathrm{H}, J=2.2 \mathrm{~Hz}), 6.86(\mathrm{~d}$, $1 \mathrm{H}, J=7.6 \mathrm{~Hz}), 6.91(\mathrm{dd}, 1 \mathrm{H}, J=8.0,2.0 \mathrm{~Hz}), 7.31(\mathrm{t}, 1 \mathrm{H}, J=7.8 \mathrm{~Hz}), 7.46(\mathrm{~s}, 1 \mathrm{H}) .{ }^{13} \mathrm{C}\left\{{ }^{1} \mathrm{H}\right\} \mathrm{NMR}$ $\left(\mathrm{CDCl}_{3}, 101 \mathrm{MHz}\right): \delta$ 54.9, 55.5, 114.0, 114.6, 120.5, 121.0, 123.8, 130.5, 135.3, 160.3. HRMS(MALDI-TOF) m/z: [M + H] ${ }^{+}$Calcd for $\mathrm{C}_{10} \mathrm{H}_{11} \mathrm{BrN}_{3} \mathrm{O}$ 268.0085; Found 268.0072.

4-Bromo-1-(o-methoxybenzyl)-1,2,3-triazole (2e): New compound.

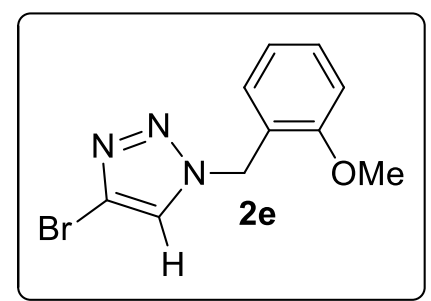

Isolated by flash chromatography (hexane/EtOAc, 2:1). Yield was 47\% (63 mg, white solid). mp: 89-90 ${ }^{\circ} \mathrm{C} .{ }^{1} \mathrm{H} \mathrm{NMR}\left(\mathrm{CDCl}_{3}, 400 \mathrm{MHz}\right)$ : $\delta 3.88(\mathrm{~s}, 3 \mathrm{H}), 5.53(\mathrm{~s}, 2 \mathrm{H}), 6.93-6.99(\mathrm{~m}, 2 \mathrm{H}), 7.25$ (dd, 1H, $J=8.0$, $2.0 \mathrm{~Hz}), 7.37(\mathrm{td}, 1 \mathrm{H}, J=8.1,1.7 \mathrm{~Hz}), 7.52(\mathrm{~s}, 1 \mathrm{H}) .{ }^{13} \mathrm{C}\left\{{ }^{1} \mathrm{H}\right\} \mathrm{NMR}$ $\left(\mathrm{CDCl}_{3}, 101 \mathrm{MHz}\right): \delta 50.0,55.6,111.0,120.3,121.2,122.3,124.0$, 130.8, 130.9, 157.3. HRMS (MALDI-TOF) m/z: $[\mathrm{M}+\mathrm{H}]^{+}$Calcd for $\mathrm{C}_{10} \mathrm{H}_{11} \mathrm{BrN}_{3} \mathrm{O} 268.0085$; Found 268.0099. 
4-Bromo-1-(naphthalen-2-ylmethyl)-1,2,3-triazole (2f): New compound.

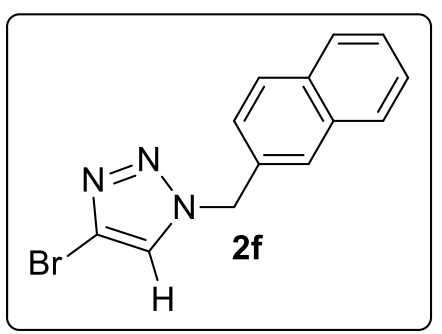

Isolated by flash chromatography (hexane/EtOAc, 2:1). Yield was $67 \%$ (97 mg, white solid). mp: $101-102{ }^{\circ} \mathrm{C} .{ }^{1} \mathrm{H}$ NMR $\left(\mathrm{CDCl}_{3}, 400\right.$ $\mathrm{MHz}): \delta 5.69(\mathrm{~s}, 2 \mathrm{H}), 7.35(\mathrm{dd}, 1 \mathrm{H}, J=8.6,1.8 \mathrm{~Hz}), 7.47(\mathrm{~s}, 1 \mathrm{H})$, $7.52-7.56(\mathrm{~m}, 2 \mathrm{H}), 7.77(\mathrm{~s}, 1 \mathrm{H}), 7.82-7.88(\mathrm{~m}, 3 \mathrm{H}) .{ }^{13} \mathrm{C}\left\{{ }^{1} \mathrm{H}\right\} \mathrm{NMR}$ $\left(\mathrm{CDCl}_{3}, 101 \mathrm{MHz}\right): \delta 55.2,121.1,123.8,125.4,127.0,127.1,127.8$, 128.0, 128.1, 129.5, 131.2, 133.3, 133.4. HRMS (MALDI-TOF) m/z: $[\mathrm{M}+\mathrm{H}]^{+}$Calcd for $\mathrm{C}_{13} \mathrm{H}_{11} \mathrm{BrN}_{3} 288.0136$; Found 288.0103.

4-Bromo-1-(2-phenylethyl)-1,2,3-triazole (2g): New compound.

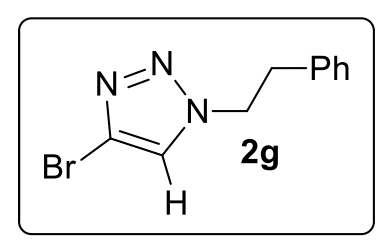

Isolated by flash chromatography (hexane/EtOAc, 2:1). Yield was 75\% (94 mg, off-white solid). mp: $72-73{ }^{\circ} \mathrm{C} .{ }^{1} \mathrm{H}$ NMR $\left(\mathrm{CDCl}_{3}, 400 \mathrm{MHz}\right): \delta$ $3.21(\mathrm{t}, 2 \mathrm{H}, J=7.0 \mathrm{~Hz}), 4.60(\mathrm{t}, 2 \mathrm{H}, J=7.0 \mathrm{~Hz}), 7.10(\mathrm{~d}, 2 \mathrm{H}, J=7.6 \mathrm{~Hz})$, 7.25-7.34 (m, 4H). ${ }^{13} \mathrm{C}\left\{{ }^{1} \mathrm{H}\right\} \mathrm{NMR}\left(\mathrm{CDCl}_{3}, 101 \mathrm{MHz}\right): \delta 36.6,52.4,120.2$, 124.1, 127.4, 128.7, 129.1, 136.7. HRMS (MALDI-TOF) m/z: $[\mathrm{M}+\mathrm{H}]^{+}$Calcd for $\mathrm{C}_{10} \mathrm{H}_{11} \mathrm{BrN}_{3}$ 252.0136; Found 252.0091.

4-Bromo-1-(3-phenyl-2-propen-1-yl)-1,2,3-triazole (2h): New compound.

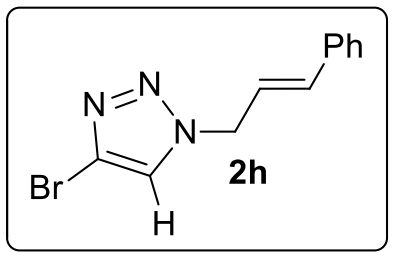

Isolated by flash chromatography (hexane/EtOAc, 2:1). Yield was 56\% (74 mg, off-white solid). mp: $77-78{ }^{\circ} \mathrm{C} .{ }^{1} \mathrm{H} \mathrm{NMR}\left(\mathrm{CDCl}_{3}, 400 \mathrm{MHz}\right): \delta$ $5.14(\mathrm{dd}, 2 \mathrm{H}, J=6.8,1.2 \mathrm{~Hz}), 6.32(\mathrm{dt}, 1 \mathrm{H}, J=16.0,6.9 \mathrm{~Hz}), 6.70(\mathrm{~d}$, $1 \mathrm{H}, J=15.6 \mathrm{~Hz}), 7.29-7.41(\mathrm{~m}, 5 \mathrm{H}), 7.62(\mathrm{~s}, 1 \mathrm{H}) .{ }^{13} \mathrm{C}\left\{{ }^{1} \mathrm{H}\right\} \mathrm{NMR}\left(\mathrm{CDCl}_{3}\right.$, $101 \mathrm{MHz}): \delta 53.2,120.9,121.1,123.6,126.9,128.9,135.3,136.4$. HRMS (MALDI-TOF) m/z: [M $+\mathrm{H}]^{+}$Calcd for $\mathrm{C}_{11} \mathrm{H}_{11} \mathrm{BrN}_{3}$ 264.0136; Found 264.0106. 


\section{Representative Procedure for Direct CuAAC of Bromo(phosphoryl)ethyne 1,}

\section{Synthesis of 1-benzyl-5-bromo-4-diphenylphosphinyl-1,2,3-triazole (3a): New}

compound.

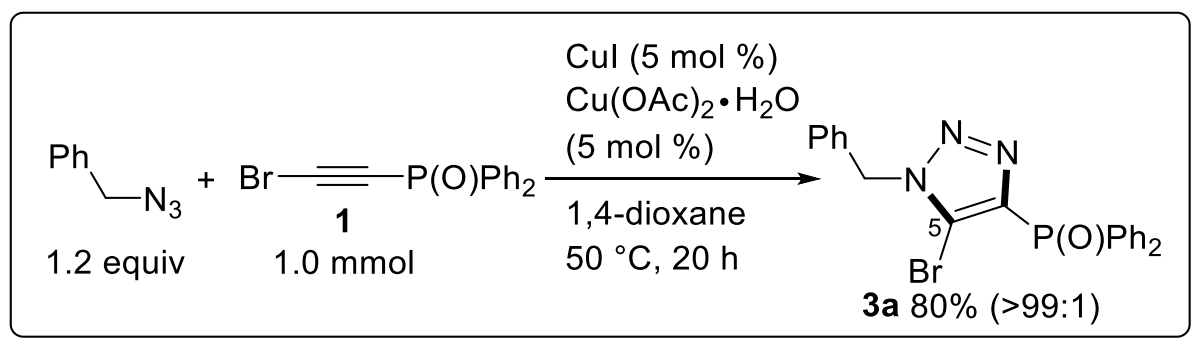

To a $50 \mathrm{~mL}$ of two-neck round-bottomed flask charged with a magnetic stirrer bar, were added bromo(phosphoryl)ethyne 1 (305 mg, $1.0 \mathrm{mmol}$ ), benzyl azide (160 mg, $1.2 \mathrm{mmol}$ ), copper iodide $(9.5 \mathrm{mg}, 0.05 \mathrm{mmol})$, copper acetate monohydrate $(10.0 \mathrm{mg}, 0.05 \mathrm{mmol})$ and 1,4-dioxane $(5 \mathrm{~mL})$. The mixture was transferred to oil bath, and stirred at $50{ }^{\circ} \mathrm{C}$ for $20 \mathrm{~h}$. After that, the resulted solution was quenched with $5 \mathrm{~mL}$ of saturated $\mathrm{NH}_{4} \mathrm{Cl}$ aq., and extracted with ethyl acetate and $\mathrm{H}_{2} \mathrm{O}$. The organic layer was dried over brine and $\mathrm{MgSO}_{4}$, and the solvents were removed under reduced pressure. The crude product was subjected to column chromatography on silica gel (hexane/EtOAc, 1:2), and the volatiles were removed under vacuum to give the corresponding 1-benzyl-5-bromo4-diphenylphosphinyl-1,2,3-triazole 3a as white solid in $80 \%$ yield (350 mg). mp: $160-162{ }^{\circ} \mathrm{C} .{ }^{1} \mathrm{H}$ NMR $\left(\mathrm{CDCl}_{3}, 400 \mathrm{MHz}\right): \delta 5.58(\mathrm{~s}, 2 \mathrm{H}), 7.28-7.37(\mathrm{~m}, 5 \mathrm{H}), 7.45-7.50(\mathrm{~m}, 4 \mathrm{H}), 7.52-7.57(\mathrm{~m}$, 2H), 7.81-7.87 (m, 4H). ${ }^{13} \mathrm{C}\left\{{ }^{1} \mathrm{H}\right\} \mathrm{NMR}\left(\mathrm{CDCl}_{3}, 101 \mathrm{MHz}\right): \delta 52.8,118.5,128.0,128.5(\mathrm{~d}, J=12.5$ $\mathrm{Hz}), 128.8,129.0,131.6$ (d, $J=111.8 \mathrm{~Hz}), 131.7$ (d, $J=10.7 \mathrm{~Hz}), 132.3(\mathrm{~d}, J=1.9 \mathrm{~Hz}), 133.2$, $139.7(\mathrm{~d}, J=134.5 \mathrm{~Hz}) .{ }^{31} \mathrm{P}\left\{{ }^{1} \mathrm{H}\right\}$ NMR $\left(\mathrm{CDCl}_{3}, 162 \mathrm{MHz}\right): \delta$ 16.3. HRMS (MALDI-TOF) m/z: $[\mathrm{M}+\mathrm{Na}]^{+} \mathrm{Calcd}$ for $\mathrm{C}_{21} \mathrm{H}_{17} \mathrm{BrN}_{3} \mathrm{NaOP} 460.0190$; Found 460.0205 . The regioselectivity of 3a was determined by the single-crystal X-ray analysis (Figure S2). We assumed that the other derivatives 3b-k would exhibit the same regioselectivity. 
5-Bromo-4-diphenylphosphinyl-1-( $p$-methoxybenzyl)-1,2,3-triazole (3b): New compound.

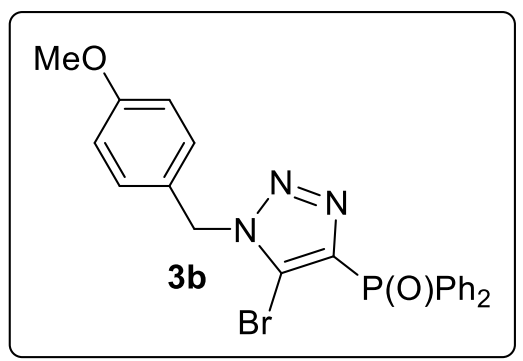

Isolated by flash chromatography (hexane/EtOAc, 1:2). Yield was $78 \%$ (733 mg, $2.0 \mathrm{mmol} \mathrm{scale}$, white solid). mp: $139-140{ }^{\circ} \mathrm{C} .{ }^{1} \mathrm{H}$ NMR (CDCl $3,400 \mathrm{MHz}): \delta 3.76(\mathrm{~s}, 3 \mathrm{H}), 5.50(\mathrm{~s}, 2 \mathrm{H}), 6.85(\mathrm{~d}, 2 \mathrm{H}$, $J=8.8 \mathrm{~Hz}), 7.26(\mathrm{~d}, 2 \mathrm{H}, J=8.4 \mathrm{~Hz}), 7.43-7.47(\mathrm{~m}, 4 \mathrm{H}), 7.50$ $7.54(\mathrm{~m}, 2 \mathrm{H}), 7.84(\mathrm{dd}, 4 \mathrm{H}, J=13.0,7.4 \mathrm{~Hz}) .{ }^{13} \mathrm{C}\left\{{ }^{1} \mathrm{H}\right\} \mathrm{NMR}$ $\left(\mathrm{CDCl}_{3}, 101 \mathrm{MHz}\right): \delta 52.4,55.3,114.4,118.2(\mathrm{~d}, J=22.2 \mathrm{~Hz})$, 125.4, 128.5 (d, $J=12.6 \mathrm{~Hz}), 129.7,131.6$ (d, $J=111.8 \mathrm{~Hz}), 131.8$ (d, $J=10.6 \mathrm{~Hz}), 132.3$ (d, $J=$ $1.9 \mathrm{~Hz}), 139.6(\mathrm{~d}, J=135.8 \mathrm{~Hz}), 160.0 .{ }^{31} \mathrm{P}\left\{{ }^{1} \mathrm{H}\right\} \mathrm{NMR}\left(\mathrm{CDCl}_{3}, 162 \mathrm{MHz}\right): \delta$ 16.2. HRMS (MALDI-TOF) m/z: [M + Na] $]^{+}$Calcd for $\mathrm{C}_{22} \mathrm{H}_{19} \mathrm{BrN}_{3} \mathrm{NaO}_{2} \mathrm{P}$ 490.0296; Found 490.0279.

5-Bromo-1-(p-bromobenzyl)-4-diphenylphosphinyl-1,2,3-triazole (3c): New compound.

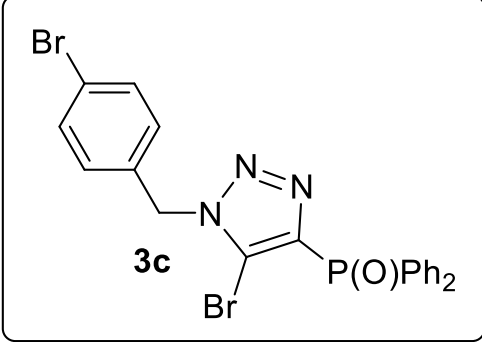

Isolated by flash chromatography (hexane/EtOAc, 1:2). Yield was $72 \%$ (374 mg, 1.0 mmol scale, white solid). mp: $168-170{ }^{\circ} \mathrm{C} .{ }^{1} \mathrm{H}$ NMR ( $\left.\mathrm{CDCl}_{3}, 400 \mathrm{MHz}\right): \delta 5.50(\mathrm{~s}, 2 \mathrm{H}), 7.16(\mathrm{~d}, 2 \mathrm{H}, J=8.4 \mathrm{~Hz})$, 7.44-7.48 (m, 6H), 7.50-7.54 (m, 2H), 7.81-7.87 (m, 4H). ${ }^{13} \mathrm{C}\left\{{ }^{1} \mathrm{H}\right\}$ NMR ( $\left.\mathrm{CDCl}_{3}, 101 \mathrm{MHz}\right): \delta 52.1,118.4(\mathrm{~d}, J=22.1 \mathrm{~Hz}), 123.0$, $128.5(\mathrm{~d}, J=12.5 \mathrm{~Hz}), 129.8,131.5(\mathrm{~d}, J=111.7 \mathrm{~Hz}), 131.7(\mathrm{~d}, J=$ $10.6 \mathrm{~Hz}), 132.18,132.22,132.3,139.9(\mathrm{~d}, J=134.9 \mathrm{~Hz}) .{ }^{31} \mathrm{P}\left\{{ }^{1} \mathrm{H}\right\} \mathrm{NMR}\left(\mathrm{CDCl}_{3}, 162 \mathrm{MHz}\right): \delta 16.2$. HRMS (MALDI-TOF) m/z: [M + H] $]^{+}$Calcd for $\mathrm{C}_{21} \mathrm{H}_{17} \mathrm{Br}_{2} \mathrm{~N}_{3} \mathrm{OP}$ 515.9476; Found 515.9468.

5-Bromo-1-(p-cyanobenzyl)-4-diphenylphosphinyl-1,2,3-triazole (3d): New compound.

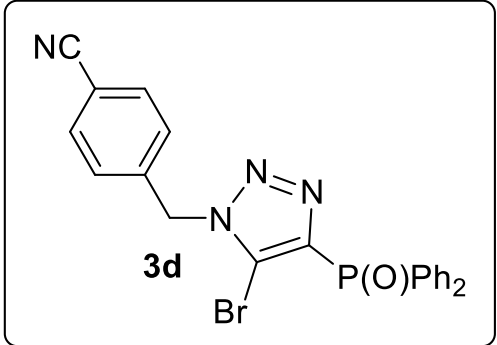

Isolated by flash chromatography (hexane/EtOAc, 1:2). Yield was 75\% (347 mg, $1.0 \mathrm{mmol}$ scale, white solid). mp: $156-157{ }^{\circ} \mathrm{C} .{ }^{1} \mathrm{H}$ NMR ( $\left.\mathrm{CDCl}_{3}, 400 \mathrm{MHz}\right): \delta 5.64(\mathrm{~s}, 2 \mathrm{H}), 7.38(\mathrm{~d}, 2 \mathrm{H}, J=8.0 \mathrm{~Hz})$, 7.46-7.50 (m, 4H), 7.53-7.58 (m, 2H), $7.65(\mathrm{~d}, 2 \mathrm{H}, J=8.8 \mathrm{~Hz})$, 7.82-7.88 (m, 4H). ${ }^{13} \mathrm{C}\left\{{ }^{1} \mathrm{H}\right\}$ NMR $\left(\mathrm{CDCl}_{3}, 101 \mathrm{MHz}\right): \delta$ 52.0, 112.9, 118.0, $118.7(\mathrm{~d}, J=22.1 \mathrm{~Hz}), 128.6(\mathrm{~d}, J=13.5 \mathrm{~Hz}), 128.7$, $131.3(\mathrm{~d}, J=111.7 \mathrm{~Hz}), 131.7(\mathrm{~d}, J=10.6 \mathrm{~Hz}), 132.4,132.8,138.2,140.1(\mathrm{~d}, J=133.9 \mathrm{~Hz})$. ${ }^{31} \mathrm{P}\left\{{ }^{1} \mathrm{H}\right\}$ NMR $\left(\mathrm{CDCl}_{3}, 162 \mathrm{MHz}\right): \delta$ 16.2. HRMS (MALDI-TOF) $\mathrm{m} / \mathrm{z}:[\mathrm{M}+\mathrm{Na}]^{+}$Calcd for $\mathrm{C}_{22} \mathrm{H}_{16} \mathrm{BrN}_{4} \mathrm{NaOP} 485.0143$; Found 485.0131. 
5-Bromo-4-diphenylphosphinyl-1-(m-methoxybenzyl)-1,2,3-triazole (3e): New compound.

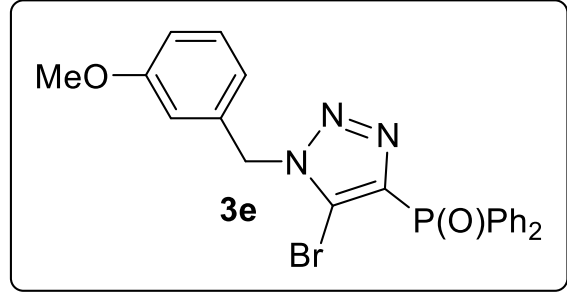

Isolated by flash chromatography (hexane/EtOAc, 1:2). Yield was $72 \%$ (677 mg, $2.0 \mathrm{mmol}$ scale, white solid). mp: 118$119{ }^{\circ} \mathrm{C} .{ }^{1} \mathrm{H}$ NMR $\left(\mathrm{CDCl}_{3}, 400 \mathrm{MHz}\right): \delta 3.74(\mathrm{~d}, 3 \mathrm{H}), 5.53(\mathrm{~s}$, $2 \mathrm{H}), 6.81-6.86(\mathrm{~m}, 3 \mathrm{H}), 7.24(\mathrm{t}, 1 \mathrm{H}, J=7.4 \mathrm{~Hz}), 7.43-7.48(\mathrm{~m}$, $4 \mathrm{H}), 7.51-7.55(\mathrm{~m}, 2 \mathrm{H}), 7.82-7.87(\mathrm{~m}, 4 \mathrm{H}) .{ }^{13} \mathrm{C}\left\{{ }^{1} \mathrm{H}\right\} \mathrm{NMR}$ $\left(\mathrm{CDCl}_{3}, 101 \mathrm{MHz}\right): \delta 52.7,55.3,113.7,114.3,118.6(\mathrm{~d}, J=22.1 \mathrm{~Hz}), 120.2,128.6(\mathrm{~d}, J=13.5$ Hz), 130.1, 131.6 (d, $J=111.8 \mathrm{~Hz}), 131.8$ (d, $J=10.6 \mathrm{~Hz}), 132.3$ (d, $J=2.9 \mathrm{~Hz}), 134.7,139.7$ (d, $J=134.8 \mathrm{~Hz}), 160.0 .{ }^{31} \mathrm{P}\left\{{ }^{1} \mathrm{H}\right\} \mathrm{NMR}\left(\mathrm{CDCl}_{3}, 162 \mathrm{MHz}\right): \delta$ 16.2. HRMS (MALDI-TOF) m/z: [M + $\mathrm{H}]^{+}$Calcd for $\mathrm{C}_{22} \mathrm{H}_{20} \mathrm{BrN}_{3} \mathrm{O}_{2} \mathrm{P}$ 468.0471; Found 468.0462.

5-Bromo-1-(m-bromobenzyl)-4-diphenylphosphinyl-1,2,3-triazole (3f): New compound.

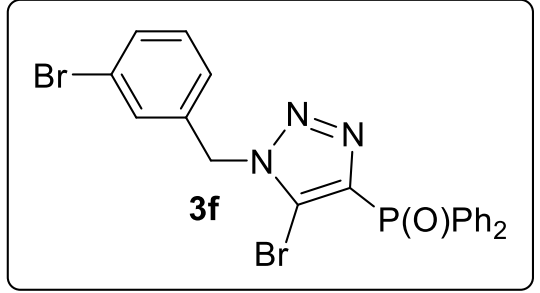

Isolated by flash chromatography (hexane/EtOAc, 1:2). Yield was $80 \%$ (823 mg, $2.0 \mathrm{mmol}$ scale, white solid). mp: $109-111^{\circ} \mathrm{C}$. ${ }^{1} \mathrm{H}$ NMR $\left(\mathrm{CDCl}_{3}, 400 \mathrm{MHz}\right): \delta 5.52(\mathrm{~s}, 2 \mathrm{H}), 7.18-7.20(\mathrm{~m}, 2 \mathrm{H})$, 7.42-7.48 (m, 6H), 7.51-7.55 (m, 2H), 7.81-7.87 (m, 4H). ${ }^{13} \mathrm{C}\left\{{ }^{1} \mathrm{H}\right\} \mathrm{NMR}\left(\mathrm{CDCl}_{3}, 101 \mathrm{MHz}\right): \delta 51.9,118.5(\mathrm{~d}, J=22.1 \mathrm{~Hz})$, $122.9,126.6,128.5(\mathrm{~d}, J=12.6 \mathrm{~Hz}), 130.6,130.97,131.02,131.4(\mathrm{~d}, J=111.8 \mathrm{~Hz}), 131.7(\mathrm{~d}, J=$ $9.7 \mathrm{~Hz}), 132.0,132.3,135.3,139.9(\mathrm{~d}, J=134.9 \mathrm{~Hz}) .{ }^{31} \mathrm{P}\left\{{ }^{1} \mathrm{H}\right\} \mathrm{NMR}\left(\mathrm{CDCl}_{3}, 162 \mathrm{MHz}\right): \delta 16.3$. HRMS (MALDI-TOF) m/z: [M + H $]^{+}$Calcd for $\mathrm{C}_{21} \mathrm{H}_{17} \mathrm{Br}_{2} \mathrm{~N}_{3} \mathrm{OP} 515.9476$; Found 515.9435.

5-Bromo-4-diphenylphosphinyl-1-(o-methoxybenzyl)-1,2,3-triazole (3g): New compound.

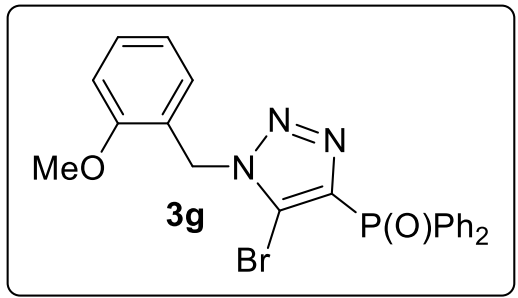

Isolated by flash chromatography (hexane/EtOAc, 1:2). Yield was $74 \%$ (697 mg, $2.0 \mathrm{mmol} \mathrm{scale,} \mathrm{white} \mathrm{solid).} \mathrm{mp}: 115-116^{\circ} \mathrm{C}$. ${ }^{1} \mathrm{H} \mathrm{NMR}\left(\mathrm{CDCl}_{3}, 400 \mathrm{MHz}\right): \delta 3.80(\mathrm{~s}, 3 \mathrm{H}), 5.59$ (s, 2H), 6.86$6.90(\mathrm{~m}, 2 \mathrm{H}), 6.96(\mathrm{dd}, 1 \mathrm{H}, J=7.4,1.4 \mathrm{~Hz}), 7.27-7.31(\mathrm{~m}, 1 \mathrm{H})$, 7.43-7.48 (m, 4H), 7.50-7.55 (m, 2H), 7.83-7.89 (m, 4H).

${ }^{13} \mathrm{C}\left\{{ }^{1} \mathrm{H}\right\} \mathrm{NMR}\left(\mathrm{CDCl}_{3}, 101 \mathrm{MHz}\right): \delta 47.9,55.4,110.6,119.0(\mathrm{~d}, J=23.1 \mathrm{~Hz}), 120.8,121.9,128.5$ (d, $J=12.6 \mathrm{~Hz}), 129.1,130.1,131.79$ (d, $J=111.8 \mathrm{~Hz}), 131.82(\mathrm{~d}, J=9.7 \mathrm{~Hz}), 132.2(\mathrm{~d}, J=2.9$ $\mathrm{Hz}), 139.2(\mathrm{~d}, J=136.8 \mathrm{~Hz}), 156.8 .{ }^{31} \mathrm{P}\left\{{ }^{1} \mathrm{H}\right\} \mathrm{NMR}\left(\mathrm{CDCl}_{3}, 162 \mathrm{MHz}\right): \delta$ 16.2. HRMS (MALDITOF) m/z: $[\mathrm{M}+\mathrm{Na}]^{+}$Calcd for $\mathrm{C}_{22} \mathrm{H}_{19} \mathrm{BrN}_{3} \mathrm{NaO}_{2} \mathrm{P} 490.0296$; Found 490.0295. 
5-Bromo-1-(o-bromobenzyl)-4-diphenylphosphinyl-1,2,3-triazole (3h): New compound.

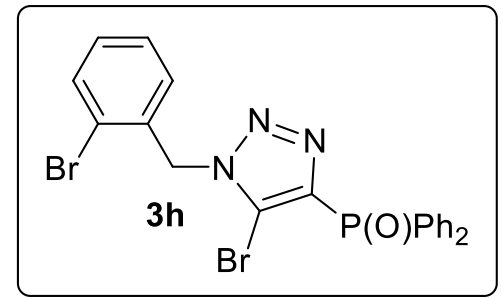

Isolated by flash chromatography (hexane/EtOAc, 1:2). Yield was $82 \%$ (848 mg, $2.0 \mathrm{mmol}$ scale, white solid). mp: $164-165{ }^{\circ} \mathrm{C} .{ }^{1} \mathrm{H}$ $\mathrm{NMR}\left(\mathrm{CDCl}_{3}, 400 \mathrm{MHz}\right): \delta 5.67(\mathrm{~s}, 2 \mathrm{H}), 6.82(\mathrm{dd}, 1 \mathrm{H}, J=7.4,1.4$ $\mathrm{Hz}), 7.16(\mathrm{td}, 1 \mathrm{H}, J=7.7,1.5 \mathrm{~Hz}), 7.22(\mathrm{td}, 1 \mathrm{H}, J=7.2,1.2 \mathrm{~Hz})$, 7.44-7.57 (m, 7H), 7.85-7.90 (m, 4H). ${ }^{13} \mathrm{C}\left\{{ }^{1} \mathrm{H}\right\} \mathrm{NMR}\left(\mathrm{CDCl}_{3}, 101\right.$ $\mathrm{MHz}): \delta 52.3,119.1(\mathrm{~d}, J=22.1 \mathrm{~Hz}), 122.5,127.9,128.4(\mathrm{~d}, J=12.6 \mathrm{~Hz}), 128.8,130.0,131.4(\mathrm{~d}$, $J=111.8 \mathrm{~Hz}), 131.6(\mathrm{~d}, J=9.5 \mathrm{~Hz}), 132.2,132.7,133.0,139.6(\mathrm{~d}, J=134.9 \mathrm{~Hz}) .{ }^{31} \mathrm{P}\left\{{ }^{1} \mathrm{H}\right\} \mathrm{NMR}$ $\left(\mathrm{CDCl}_{3}, 162 \mathrm{MHz}\right): \delta$ 16.1. HRMS (MALDI-TOF) m/z: $[\mathrm{M}+\mathrm{Na}]^{+}$Calcd for $\mathrm{C}_{21} \mathrm{H}_{16} \mathrm{Br}_{2} \mathrm{~N}_{3} \mathrm{NaOP}$ 537.9295; Found 537.9318.

5-Bromo-4-diphenylphosphinyl-1-(2-phenylethyl)-1,2,3-triazole (3i): New compound.

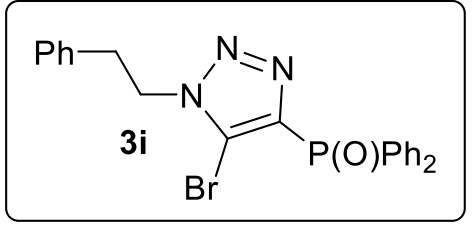

Isolated by flash chromatography (hexane/EtOAc, 1:2). Yield was $74 \%$ (334 mg, white solid). mp: $163-164{ }^{\circ} \mathrm{C} .{ }^{1} \mathrm{H}$ NMR $\left(\mathrm{CDCl}_{3}, 400\right.$ $\mathrm{MHz}): \delta 3.22(\mathrm{t}, 2 \mathrm{H}, J=7.6 \mathrm{~Hz}), 4.61(\mathrm{t}, 2 \mathrm{H}, J=7.6 \mathrm{~Hz}), 7.10-7.12$ (m, 2H), 7.24-7.28 (m, 3H), 7.46-7.50 (m, 4H), 7.54-7.58 (m, 2H), 7.79-7.84 (m, 4H). ${ }^{13} \mathrm{C}\left\{{ }^{1} \mathrm{H}\right\} \mathrm{NMR}\left(\mathrm{CDCl}_{3}, 101 \mathrm{MHz}\right): \delta 36.0,50.3,118.7(\mathrm{~d}, J=23.1 \mathrm{~Hz}), 127.4$, $128.6(\mathrm{~d}, J=12.6 \mathrm{~Hz}), 128.8,128.9,131.7(\mathrm{~d}, J=111.8 \mathrm{~Hz}), 131.9(\mathrm{~d}, J=10.7 \mathrm{~Hz}), 132.4(\mathrm{~d}, J=$ $2.9 \mathrm{~Hz}), 136.2,139.2(\mathrm{~d}, J=135.8 \mathrm{~Hz}) .{ }^{31} \mathrm{P}\left\{{ }^{1} \mathrm{H}\right\} \mathrm{NMR}\left(\mathrm{CDCl}_{3}, 162 \mathrm{MHz}\right): \delta$ 16.6. HRMS $\left(\right.$ MALDI-TOF) m/z: $[\mathrm{M}+\mathrm{Na}]^{+}$Calcd for $\mathrm{C}_{22} \mathrm{H}_{19} \mathrm{BrN}_{3} \mathrm{NaOP} 474.0347$; Found 474.0392.

5-Bromo-1-cyclohexyl-4-diphenylphosphinyl-1,2,3-triazole (3j): New compound.

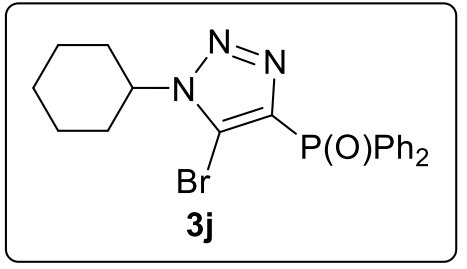

Isolated by flash chromatography (hexane/EtOAc, 1:1). Yield was 13\% (55 mg, white solid). mp: $165-167{ }^{\circ} \mathrm{C} .{ }^{1} \mathrm{H}$ NMR $\left(\mathrm{CDCl}_{3}, 400\right.$ $\mathrm{MHz}): \delta 1.30-1.50(\mathrm{~m}, 3 \mathrm{H}), 1.76(\mathrm{~d}, 1 \mathrm{H}, J=12.4 \mathrm{~Hz}), 1.94-2.10(\mathrm{~m}$, $6 \mathrm{H}), 4.34-4.42(\mathrm{~m}, 1 \mathrm{H}), 7.46-7.50(\mathrm{~m}, 4 \mathrm{H}), 7.53-7.57(\mathrm{~m}, 2 \mathrm{H}), 7.86$ $(\mathrm{dd}, 4 \mathrm{H}, J=12.8,7.2 \mathrm{~Hz}) .{ }^{13} \mathrm{C}\left\{{ }^{1} \mathrm{H}\right\} \mathrm{NMR}\left(\mathrm{CDCl}_{3}, 101 \mathrm{MHz}\right): \delta 25.0,25.4,32.5,59.8,117.5(\mathrm{~d}, J$ $=22.1 \mathrm{~Hz}), 128.6(\mathrm{~d}, J=12.6 \mathrm{~Hz}), 131.8(\mathrm{~d}, J=111.7 \mathrm{~Hz}), 131.9$ (d, $J=9.5 \mathrm{~Hz}), 132.3$ (d, $J=2.0$ $\mathrm{Hz}), 138.9$ (d, $J=136.8 \mathrm{~Hz}) .{ }^{31} \mathrm{P}\left\{{ }^{1} \mathrm{H}\right\} \mathrm{NMR}\left(\mathrm{CDCl}_{3}, 162 \mathrm{MHz}\right): \delta$ 16.6. HRMS (MALDI-TOF) $\mathrm{m} / \mathrm{z}:[\mathrm{M}+\mathrm{H}]^{+}$Calcd for $\mathrm{C}_{20} \mathrm{H}_{22} \mathrm{BrN}_{3} \mathrm{OP} 430.0678$; Found 430.0698 . 
5-Bromo-1-(p-bromophenyl)-4-diphenylphosphinyl-1,2,3-triazole (3k): New compound.

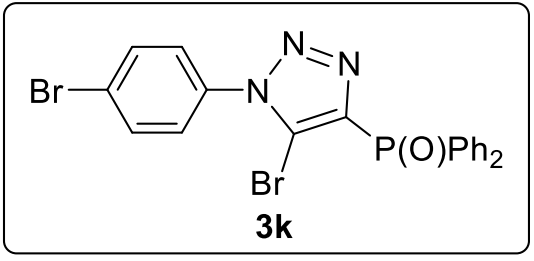

Isolated by flash chromatography (hexane/EtOAc, 1:2). Yield was $48 \%$ (241 mg, white solid). mp: 196-197 ${ }^{\circ} \mathrm{C}$. ${ }^{1} \mathrm{H}$ NMR $\left(\mathrm{CDCl}_{3}, 400 \mathrm{MHz}\right): \delta 7.44(\mathrm{~d}, 2 \mathrm{H}, J=8.4 \mathrm{~Hz}), 7.50-7.54(\mathrm{~m}$, $4 \mathrm{H}), 7.57-7.62(\mathrm{~m}, 2 \mathrm{H}), 7.71(\mathrm{~d}, 2 \mathrm{H}, J=8.8 \mathrm{~Hz}), 7.89-7.95(\mathrm{~m}$,

4H). ${ }^{13} \mathrm{C}\left\{{ }^{1} \mathrm{H}\right\} \mathrm{NMR}\left(\mathrm{CDCl}_{3}, 101 \mathrm{MHz}\right): \delta 118.9(\mathrm{~d}, J=22.1 \mathrm{~Hz}), 124.9,127.3,128.7(\mathrm{~d}, J=12.5$ Hz), 131.4 (d, $J=110.8 \mathrm{~Hz}), 131.9$ (d, $J=10.7 \mathrm{~Hz}), 132.6$ (d, $J=2.9 \mathrm{~Hz}), 132.9,133.9,140.4$ (d, $J=133.9 \mathrm{~Hz}) .{ }^{31} \mathrm{P}\left\{{ }^{1} \mathrm{H}\right\} \mathrm{NMR}\left(\mathrm{CDCl}_{3}, 162 \mathrm{MHz}\right): \delta$ 16.3. HRMS (MALDI-TOF) m/z: [M + Na] ${ }^{+}$ Calcd for $\mathrm{C}_{20} \mathrm{H}_{14} \mathrm{Br}_{2} \mathrm{~N}_{3} \mathrm{NaOP}$ 523.9133; Found 523.9146. 


\section{Representative Procedure for Palladium-Catalyzed Amination of 4-Bromotriazole 2a,}

Synthesis of 1-benzyl-4-( $N$-methyl- $N$-phenylamino)-1,2,3-triazole (5a): New compound.

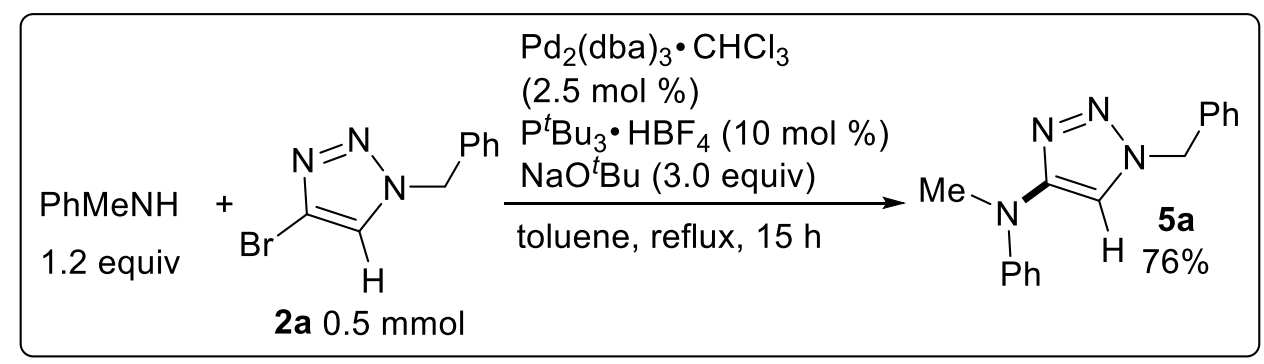

To a $50 \mathrm{~mL}$ of two-neck round-bottomed flask charged with a magnetic stirrer bar, were added 4-bromotriazole 2a (119 mg, $0.5 \mathrm{mmol}), \mathrm{Pd}_{2}(\mathrm{dba})_{3}{ }^{\circ} \mathrm{CHCl}_{3}(12.9 \mathrm{mg}, 0.0125 \mathrm{mmol})$, tri-tertbutylphosphonium tetrafluoroborate (14.5 mg, $0.05 \mathrm{mmol}$ ), sodium tert-butoxide (144 mg, 1.5 mmol), $N$-methylaniline $(64.3 \mathrm{mg}, 0.6 \mathrm{mmol})$ and toluene $(5 \mathrm{~mL})$. The mixture was transferred to oil bath, and stirred at reflux for $15 \mathrm{~h}$. After that, the resulted solution was quenched with $5 \mathrm{~mL}$ of saturated $\mathrm{NH}_{4} \mathrm{Cl}$ aq., and extracted with ethyl acetate and $\mathrm{H}_{2} \mathrm{O}$. The organic layer was dried over brine and $\mathrm{MgSO}_{4}$, and the solvents were removed under reduced pressure. The crude product was subjected to column chromatography on silica gel (hexane/EtOAc, 2:1), and the volatiles were removed under vacuum to give the corresponding 1-benzyl-4-( $N$-methyl- $N$-phenylamino)-1,2,3triazole (5a) as white solid in 76\% yield $(100 \mathrm{mg}) .{ }^{1} \mathrm{H}$ NMR $\left(\mathrm{CDCl}_{3}, 400 \mathrm{MHz}\right): \delta 3.41(\mathrm{~s}, 3 \mathrm{H})$, $5.45(\mathrm{~s}, 2 \mathrm{H}), 6.91(\mathrm{t}, 1 \mathrm{H}, J=7.0 \mathrm{~Hz}), 7.07(\mathrm{~d}, 2 \mathrm{H}, J=8.0 \mathrm{~Hz}), 7.09(\mathrm{~s}, 1 \mathrm{H}), 7.23-7.28(\mathrm{~m}, 4 \mathrm{H})$, 7.33-7.37 (m, 3H). ${ }^{13} \mathrm{C}\left\{{ }^{1} \mathrm{H}\right\}$ NMR $\left(\mathrm{CDCl}_{3}, 101 \mathrm{MHz}\right): \delta 38.6,54.7,111.0,118.0,121.2,128.1$, 128.8, 129.19, 129.23, 134.9, 147.6, 154.0. HRMS (MALDI-TOF) $\mathrm{m} / \mathrm{z}:[\mathrm{M}+\mathrm{H}]^{+}$Calcd for $\mathrm{C}_{16} \mathrm{H}_{17} \mathrm{~N}_{4} 265.1453$; Found 265.1461. 


\section{Representative Procedure for Tandem Sulfenylation and Dephosphorylation,}

Synthesis of 1-benzyl-5-(p-tolylthio)-1,2,3-triazole (7a): Known compound, CAS RN [1654724-32-9]. ${ }^{21}$

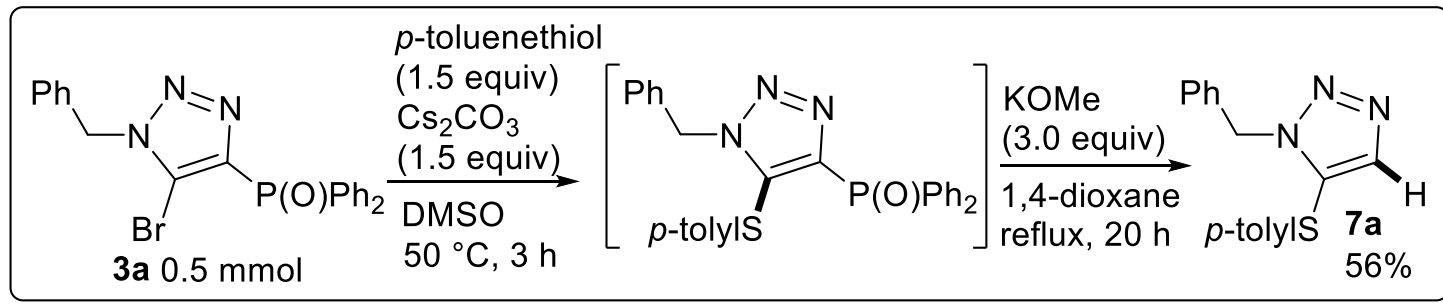

To a $50 \mathrm{~mL}$ of two-neck round-bottomed flask charged with a magnetic stirrer bar, were added 5-bromo-4-phosphoryl-1,2,3-triazole 3a (219 mg, $0.5 \mathrm{mmol}$ ), $p$-toluenethiol (93 mg, $0.75 \mathrm{mmol}$ ), cesium carbonate ( $244 \mathrm{mg}, 0.75 \mathrm{mmol}$ ) and DMSO $(5 \mathrm{~mL})$. The mixture was transferred to oil bath, and stirred at $50{ }^{\circ} \mathrm{C}$ for $3 \mathrm{~h}$. After that, the resulted solution was quenched with $5 \mathrm{~mL}$ of saturated $\mathrm{NH}_{4} \mathrm{Cl}$ aq., and extracted with ethyl acetate and $\mathrm{H}_{2} \mathrm{O}$. The organic layer was dried over brine and $\mathrm{MgSO}_{4}$, and the solvents were removed under reduced pressure. Subsequently, $50 \mathrm{~mL}$ of singleneck round bottomed flask with a magnetic stir bar and crude product, were added KOMe (105 mg, $1.5 \mathrm{mmol})$ and 1,4-dioxane $(5 \mathrm{~mL})$. The mixture stirred at reflux $\left(\sim 110{ }^{\circ} \mathrm{C}\right)$ for $20 \mathrm{~h}$, after a consumption of starting triazole that determined by TLC analysis, the resulted solution was quenched with $5 \mathrm{~mL}$ of saturated $\mathrm{NH}_{4} \mathrm{Cl}$ aq., and extracted with ethyl acetate and $\mathrm{H}_{2} \mathrm{O}$. The organic layer was dried over brine and $\mathrm{MgSO}_{4}$, and the solvents were removed under reduced pressure. The crude product was subjected to column chromatography on silica gel (hexane/EtOAc, 3:1), and the volatiles were removed under vacuum to give the corresponding 1-benzyl-5-( $p$-tolylthio)1,2,3-triazole (7a) as white solid in $56 \%$ yield $(79 \mathrm{mg}) .{ }^{1} \mathrm{H} \mathrm{NMR}\left(\mathrm{CDCl}_{3}, 400 \mathrm{MHz}\right): \delta 2.28$ (s, $3 \mathrm{H}), 5.50(\mathrm{~s}, 2 \mathrm{H}), 6.96(\mathrm{~d}, 2 \mathrm{H}, J=8.4 \mathrm{~Hz}), 7.10(\mathrm{~d}, 2 \mathrm{H}, J=8.0 \mathrm{~Hz}), 7.16-7.19(\mathrm{~m}, 2 \mathrm{H}), 7.22-7.26$ $(\mathrm{m}, 3 \mathrm{H}), 7.76(\mathrm{~s}, 1 \mathrm{H}) .{ }^{13} \mathrm{C}\left\{{ }^{1} \mathrm{H}\right\} \mathrm{NMR}\left(\mathrm{CDCl}_{3}, 101 \mathrm{MHz}\right): \delta 21.0,51.9,127.9,128.2,128.7,129.05$ (br), 129.14, 129.3, 130.3, 134.6, 137.8, 139.3. HRMS (MALDI-TOF) m/z: $[\mathrm{M}+\mathrm{H}]^{+}$Calcd for $\mathrm{C}_{16} \mathrm{H}_{16} \mathrm{~N}_{3} \mathrm{~S} 282.1065$; Found 282.1043.

1-Benzyl-5-(p-bromophenylthio)-1,2,3-triazole
compound, $\mathrm{CAS} \mathrm{RN}$ [1654724-33-0].
Isolated by flash chromatography (hexane/EtOAc, 3:1). Yield was $49 \%$
(85 mg, white solid). ${ }^{2} \mathrm{H} \mathrm{NMR}\left(\mathrm{CDCl}_{3}, 400 \mathrm{MHz}\right): \delta 5.53(\mathrm{~s}, 2 \mathrm{H}), 6.78$


$(\mathrm{d}, 2 \mathrm{H}, J=8.8 \mathrm{~Hz}), 7.13(\mathrm{~d}, 2 \mathrm{H}, J=7.2 \mathrm{~Hz}), 7.18-7.26(\mathrm{~m}, 5 \mathrm{H}), 7.85(\mathrm{~s}, 1 \mathrm{H}) .{ }^{13} \mathrm{C}\left\{{ }^{1} \mathrm{H}\right\} \mathrm{NMR}$ $\left(\mathrm{CDCl}_{3}, 101 \mathrm{MHz}\right): \delta$ 52.2, 121.4, 127.2, 128.0, 128.4, 128.9, 129.7, 132.3, 132.5, 134.3, 140.4 . HRMS (MALDI-TOF) m/z: $[\mathrm{M}+\mathrm{H}]^{+}$Calcd for $\mathrm{C}_{15} \mathrm{H}_{13} \mathrm{BrN}_{3} \mathrm{~S}$ 346.0014; Found 346.0036.

1-Benzyl-5-( $m, p$-dimethoxyphenylthio)-1,2,3-triazole (7c): New compound.

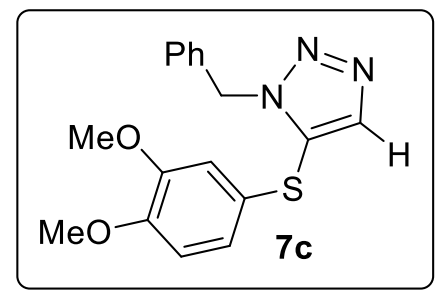

Isolated by flash chromatography (hexane/EtOAc, 7:3). Yield was 56\% (91 mg, white solid). ${ }^{1} \mathrm{H}$ NMR ( $\left.\mathrm{CDCl}_{3}, 400 \mathrm{MHz}\right): \delta 3.71$ (s, 3H), $3.84(\mathrm{~s}, 3 \mathrm{H}), 5.54(\mathrm{~s}, 2 \mathrm{H}), 6.57(\mathrm{~d}, 1 \mathrm{H}, J=1.6 \mathrm{~Hz}), 6.70-6.77(\mathrm{~m}, 2 \mathrm{H})$, 7.17-7.19 (m, 2H), 7.26-7.27 (m, 3H), $7.71(\mathrm{~s}, 1 \mathrm{H}) .{ }^{13} \mathrm{C}\left\{{ }^{1} \mathrm{H}\right\} \mathrm{NMR}$ $\left(\mathrm{CDCl}_{3}, 101 \mathrm{MHz}\right): \delta 52.0,56.0,56.1,111.9,113.5,123.0,123.5$, 127.9, 128.4, 128.8, 130.2, 134.7, 138.7, 149.4, 149.7. HRMS (MALDI-TOF) m/z: $[\mathrm{M}+\mathrm{H}]^{+}$Calcd for $\mathrm{C}_{17} \mathrm{H}_{18} \mathrm{~N}_{3} \mathrm{O}_{2} \mathrm{~S} 328.1120$; Found 328.1135 .

1-Benzyl-5-(1-decylthio)-1,2,3-triazole (7d): New compound.

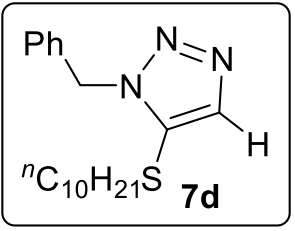

Isolated by flash chromatography (hexane/EtOAc, 3:1). Yield was 56\% (93 $\mathrm{mg}$, white solid). Prior column purification was required before $\mathrm{KOMe}-$ mediated dephosphorylation. ${ }^{1} \mathrm{H}$ NMR $\left(\mathrm{CDCl}_{3}, 400 \mathrm{MHz}\right): \delta 0.88$ (t, 3H, $J=$ $6.8 \mathrm{~Hz}$ ), 1.21-1.32 (m, 14H), 1.45 (quin, $2 \mathrm{H}, J=7.4 \mathrm{~Hz}$ ), $2.52(\mathrm{t}, 2 \mathrm{H}, J=7.0$

$\mathrm{Hz}), 5.59$ (s, 2H), 7.26-7.35 (m, 5H), $7.67(\mathrm{~s}, 1 \mathrm{H}) .{ }^{13} \mathrm{C}\left\{{ }^{1} \mathrm{H}\right\} \mathrm{NMR}\left(\mathrm{CDCl}_{3}, 101 \mathrm{MHz}\right): \delta 14.2,22.8$, 28.5, 29.1, 29.3, 29.4, 29.5, 29.6, 32.0, 36.0, 51.9, 127.9, 128.4, 129.0, 130.8, 135.2, 137.7. HRMS (MALDI-TOF) m/z: [M + H] $]^{+}$Calcd for $\mathrm{C}_{19} \mathrm{H}_{30} \mathrm{~N}_{3} \mathrm{~S}$ 332.2160; Found 332.2176.

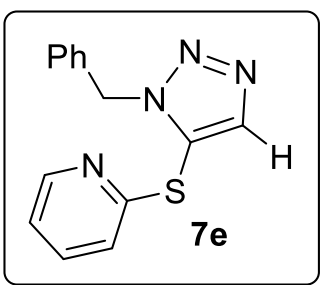

1-Benzyl-5-(2-pyridylthio)-1,2,3-triazole (7e): New compound.

Isolated by flash chromatography (hexane/EtOAc, 7:3). Yield was 35\% (47 mg, off-white solid). ${ }^{1} \mathrm{H}$ NMR $\left(\mathrm{CDCl}_{3}, 400 \mathrm{MHz}\right): \delta 5.60$ (s, $\left.2 \mathrm{H}\right), 6.57$ (d, $1 \mathrm{H}, J=8.0 \mathrm{~Hz}), 7.00(\mathrm{dd}, 1 \mathrm{H}, J=7.4,2.6 \mathrm{~Hz}), 7.17-7.24(\mathrm{~m}, 5 \mathrm{H}), 7.31-7.36$ $(\mathrm{m}, 1 \mathrm{H}), 7.95(\mathrm{~s}, 1 \mathrm{H}), 8.32(\mathrm{~d}, 1 \mathrm{H}, J=4.4 \mathrm{~Hz}) \cdot{ }^{13} \mathrm{C}\left\{{ }^{1} \mathrm{H}\right\} \mathrm{NMR}\left(\mathrm{CDCl}_{3}, 101\right.$ MHz): $\delta 52.4,120.7,121.0,125.1,128.28,128.33,128.7,134.4,137.1,141.3,149.9,156.6$. HRMS (MALDI-TOF) m/z: [M + H] $]^{+}$Calcd for $\mathrm{C}_{14} \mathrm{H}_{13} \mathrm{~N}_{4} \mathrm{~S}$ 269.0861; Found 269.0832. 


\section{Representative Procedures for Synthesis of NCC-149 via Dephosphorylative CuAAC of Bromo(phosphoryl)ethyne 1}

[Step 1] Synthesis of 4-bromo-1-(phenylthiomethyl)-1,2,3-triazole (2j): New compound.

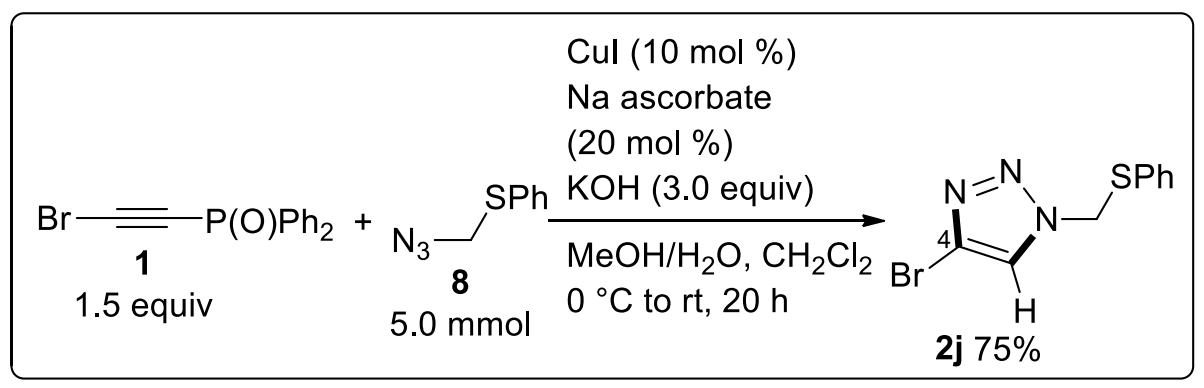

To a $100 \mathrm{~mL}$ of two-neck round-bottomed flask charged with a magnetic stirrer bar, were added bromo(phosphoryl)ethyne 1 (2.288 g, $7.5 \mathrm{mmol}$ ), copper iodide (95 mg, $0.5 \mathrm{mmol}$ ), sodium ascorbate (198 mg, $1.0 \mathrm{mmol}), \mathrm{KOH}(842 \mathrm{mg}, 15 \mathrm{mmol})$ and $\mathrm{CH}_{2} \mathrm{Cl}_{2}(50 \mathrm{~mL})$. The mixture was stirred at $0{ }^{\circ} \mathrm{C}$ for $5 \mathrm{~min}$, then phenylthiomethyl azide ( $826 \mathrm{mg}, 5.0 \mathrm{mmol}$ ) was added. After that, the mixture was warmed up to room temperature and stirred for $20 \mathrm{~h}$. The resulted mixture was extracted with ethyl acetate and $\mathrm{H}_{2} \mathrm{O}$, and dried over brine and $\mathrm{MgSO}_{4}$. The crude product was purified by flash chromatography (hexane/EtOAc, 3:1) to afford the corresponding 4bromotriazole $2 \mathbf{j}$ in $75 \%$ yield (1.013 g, off-white solid). mp: 60-61 ${ }^{\circ} \mathrm{C} .{ }^{1} \mathrm{H} \mathrm{NMR}\left(\mathrm{CDCl}_{3}, 400\right.$ $\mathrm{MHz}): \delta 5.61(\mathrm{~s}, 2 \mathrm{H}), 7.33(\mathrm{~s}, 5 \mathrm{H}), 7.59(\mathrm{~s}, 1 \mathrm{H}) .{ }^{13} \mathrm{C}\left\{{ }^{1} \mathrm{H}\right\} \mathrm{NMR}\left(\mathrm{CDCl}_{3}, 101 \mathrm{MHz}\right): \delta$ 54.7, 121.0, 123.4, 129.1, 129.7, 131.4, 132.3. HRMS (MALDI-TOF) m/z: $[\mathrm{M}+\mathrm{H}]^{+}$Calcd for $\mathrm{C}_{9} \mathrm{H}_{9} \mathrm{BrN}_{3} \mathrm{~S}$ 269.9701; Found 269.9710. 


\section{[Step 2] Palladium-Catalyzed Suzuki-Miyaura Coupling of 4-Bromotriazole 2j}

\section{Synthesis of 4-(m-Methoxycarbonylbenzene)-1-(phenylthiomethyl)-1,2,3-triazole (10):}

New compound.

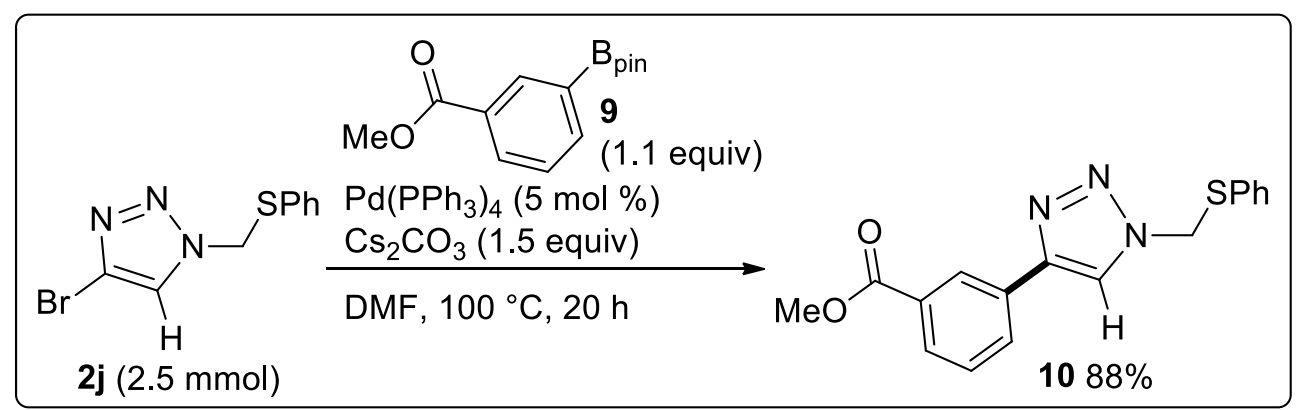

To a $100 \mathrm{~mL}$ of two-neck round-bottomed flask charged with a magnetic stirrer bar, were added 4-bromotriazole $2 \mathbf{j}$ (675 mg, $2.5 \mathrm{mmol}), \mathrm{Pd}\left(\mathrm{PPh}_{3}\right)_{4}$ (144 mg, $0.125 \mathrm{mmol}$ ), methyl 3-(4,4,5,5tetramethyl-1,3,2-dioxaborolan-2-yl)benzoate 9 (721 mg, $2.75 \mathrm{mmol})$, cesium carbonate $(1.222 \mathrm{~g}$, $3.75 \mathrm{mmol}$ ) and DMF $(25 \mathrm{~mL})$. The mixture was stirred at $100{ }^{\circ} \mathrm{C}$ for $24 \mathrm{~h}$. The resulted mixture was extracted with ethyl acetate and $\mathrm{H}_{2} \mathrm{O}$, and dried over brine and $\mathrm{MgSO}_{4}$. The crude product was purified by flash chromatography (hexane/EtOAc, $3: 1$ to 3:2) to afford the corresponding product 10 in $88 \%$ yield (719 mg, white solid). mp: $\left.91-92{ }^{\circ} \mathrm{C} .{ }^{1} \mathrm{H} \mathrm{NMR} \mathrm{(CDCl} 3,400 \mathrm{MHz}\right): \delta 3.94(\mathrm{~s}, 3 \mathrm{H})$, $5.68(\mathrm{~s}, 2 \mathrm{H}), 7.31-7.38(\mathrm{~m}, 5 \mathrm{H}), 7.50(\mathrm{t}, 1 \mathrm{H}, J=7.8 \mathrm{~Hz}), 7.88$, (s, $1 \mathrm{H}), 8.00(\mathrm{dt}, 1 \mathrm{H}, J=7.6,1.4$ $\mathrm{Hz}), 8.07(\mathrm{dt}, 1 \mathrm{H}, J=7.6,1.4 \mathrm{~Hz}), 8.38(\mathrm{t}, 1 \mathrm{H}, J=1.6 \mathrm{~Hz}) .{ }^{13} \mathrm{C}\left\{{ }^{1} \mathrm{H}\right\} \mathrm{NMR}\left(\mathrm{CDCl}_{3}, 101 \mathrm{MHz}\right): \delta$ 52.4, 54.1, 119.5, 126.9, 128.9, 129.2, 129.4, 129.7, 130.2, 130.9, 131.9, 132.3, 147.5, 166.9 . HRMS (MALDI-TOF) m/z: [M + H] $]^{+}$Calcd for $\mathrm{C}_{17} \mathrm{H}_{16} \mathrm{~N}_{3} \mathrm{O}_{2} \mathrm{~S} 326.0963$; Found 326.0976. 
[Step 3] Hydroxamination of Methyl Ester, Synthesis of NCC-149: Known compound. ${ }^{22}$

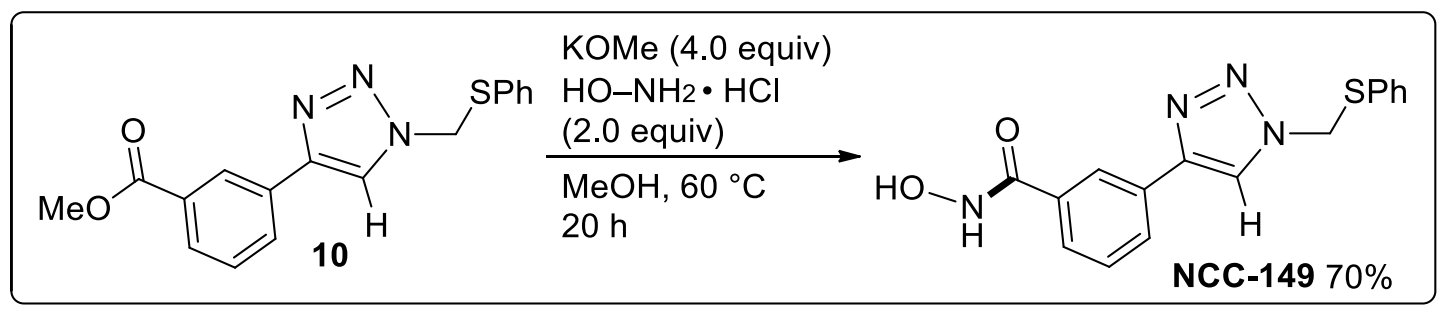

To a $100 \mathrm{~mL}$ of two-neck round-bottomed flask charged with a magnetic stirrer bar, were added methyl ester 10 (601 mg, $1.85 \mathrm{mmol}$ ), hydroxylamine hydrochloride (256 mg, $3.69 \mathrm{mmol}$ ), KOMe (518 mg, $7.39 \mathrm{mmol})$, anhydrous $\mathrm{MeOH}(20 \mathrm{~mL})$. The mixture was stirred at $60^{\circ} \mathrm{C}$ for $20 \mathrm{~h}$. After that, the resulted solution was quenched with acetic acid $(2 \mathrm{~mL})$. The resulted mixture was extracted with ethyl acetate and saturated $\mathrm{NaCl}$ aq., and dried over $\mathrm{MgSO}_{4}$. The crude product was purified by flash chromatography (hexane/EtOAc, 1:2) to afford the corresponding NCC-149 in 70\% yield (420 mg, white solid). ${ }^{1} \mathrm{H}$ NMR (acetone- $d_{6}, 400 \mathrm{MHz}$ ): $\delta 5.95$ (s, 2H), 7.33-7.38 (m, 3H), 7.45$7.48(\mathrm{~m}, 2 \mathrm{H}), 7.53(\mathrm{t}, 1 \mathrm{H}, J=7.8 \mathrm{~Hz}), 7.80(\mathrm{dt}, 1 \mathrm{H}, J=8.0,1.6 \mathrm{~Hz}), 8.02(\mathrm{dt}, 1 \mathrm{H}, J=8.4,1.3 \mathrm{~Hz})$, $8.31(\mathrm{t}, 1 \mathrm{H}, J=1.4 \mathrm{~Hz}), 8.45(\mathrm{~s}, 1 \mathrm{H}), \mathrm{N}-\mathrm{H}$ and $\mathrm{O}-\mathrm{H}$ protons could not be detected. ${ }^{13} \mathrm{C}\left\{{ }^{1} \mathrm{H}\right\}$ NMR (acetone- $d_{6}, 101 \mathrm{MHz}$ ): $\delta 53.1,120.7,123.9,126.4,128.2,128.3,129.1,129.4,131.4,131.8,132.6$, 133.0, 146.8 . 


\section{Optimization of Dephosphorylative and Direct CuAACs}

Table S1. Optimization of reaction conditions on dephosphorylative CuAAC of $\mathbf{1 .}$

\begin{tabular}{|c|c|c|c|c|c|}
\hline \multirow[b]{2}{*}{ entry $^{a}$} & $\begin{array}{l}\overline{=} \\
1(1.5 \mathrm{eq}\end{array}$ & O) $\mathrm{Ph}_{2}+\underset{\mathrm{N}_{3}}{0.5 \mathrm{mmol}} \frac{\mathrm{Ph}}{\mathrm{pr}}$ & $\begin{array}{l}(10 \mathrm{~mol} \%) \\
\text { ascorbate }(x \mathrm{mc} \\
-(3.0 \text { equiv }) \\
\text { ton source, so } \\
\text { p., } 20 \mathrm{~h}\end{array}$ & $\underset{\text { vent }}{\longrightarrow}$ & $\int_{\mathrm{H}}^{\mathrm{N}-\mathrm{Ph}}$ \\
\hline & solvent & $\begin{array}{l}\text { proton source } \\
\text { (y equiv) }\end{array}$ & $\begin{array}{c}\text { Na ascorbate } \\
(\mathbf{x} \text { mol \%) }\end{array}$ & temp. & NMR yield (\%) \\
\hline 1 & toluene & $\mathrm{MeOH}(4.0)$ & 0 & $\mathrm{rt}$ & 33 \\
\hline 2 & $\mathrm{CH}_{2} \mathrm{Cl}_{2}$ & $\mathrm{MeOH}(4.0)$ & 0 & $\mathrm{rt}$ & 55 \\
\hline 3 & THF & $\mathrm{MeOH}(4.0)$ & 0 & $\mathrm{rt}$ & 15 \\
\hline 4 & NMP & $\mathrm{MeOH}(4.0)$ & 0 & $\mathrm{rt}$ & 24 \\
\hline $5^{b}$ & $\mathrm{MeOH}$ & - & 0 & $\mathrm{rt}$ & 4 \\
\hline 6 & $\mathrm{CH}_{2} \mathrm{Cl}_{2}$ & $\mathrm{MeOH}(4.0) / \mathrm{H}_{2} \mathrm{O}(2.0)$ & 0 & $\mathrm{rt}$ & 58 \\
\hline 7 & $\mathrm{CH}_{2} \mathrm{Cl}_{2}$ & $\mathrm{MeOH}(4.0) / \mathrm{H}_{2} \mathrm{O}(2.0)$ & 20 & $\mathrm{rt}$ & 66 \\
\hline 8 & $\mathrm{CH}_{2} \mathrm{Cl}_{2}$ & $\mathrm{MeOH}(4.0) / \mathrm{H}_{2} \mathrm{O}(2.0)$ & 20 & $0{ }^{\circ} \mathrm{C}$ to $\mathrm{rt}$ & $81(75)^{c}$ \\
\hline
\end{tabular}

${ }^{a}$ Reaction conditions: $1(0.75 \mathrm{mmol})$, azide $(0.5 \mathrm{mmol})$, Cul $(10 \mathrm{~mol} \%), \mathrm{Na}$ ascorbate (x mol\%), $\mathrm{KOH}(1.5 \mathrm{mmol})$, proton source (y equiv) in solvent $(5 \mathrm{~mL})$ at temp. $\left({ }^{\circ} \mathrm{C}\right)$ for $20 \mathrm{~h} .{ }^{b} \mathrm{MeOH}\left(5 \mathrm{~mL}\right.$ ) was added without additional proton source. ${ }^{c}$ An isolated yield. 
Table S2. Optimization of reaction conditions on direct CuAAC of $\mathbf{1 .}$

\begin{tabular}{|c|c|c|c|c|c|c|}
\hline$\underset{1.2 \text { equiv }}{\mathrm{Ph}_{\mathrm{N}_{3}}}+$ & $\mathrm{Br} \frac{\overline{\overline{1}}}{\sqrt{1}} \mathrm{~F}$ & $\mathrm{Ph}_{2}$ & $\begin{array}{l}\text { Cul (x mo } \\
\mathrm{Cu}(\mathrm{OAc})_{2} \\
(\mathbf{y} \text { mol \% }\end{array}$ & $\stackrel{\mathrm{O})}{\longrightarrow} \stackrel{\mathrm{Ph}}{\longrightarrow}$ & $=_{3 a}{ }^{+}(\mathrm{O}) \mathrm{Ph}_{2}$ & ${ }_{3 a^{\prime}}{ }_{P(O) P_{2}}^{N=N}$ \\
\hline entry $^{a}$ & $\begin{array}{c}\text { Cul } \\
(x \text { mol \%) }\end{array}$ & $\begin{array}{l}\mathrm{Cu}(\mathrm{O} / \\
(\mathbf{y} \mathbf{r}\end{array}$ & $\begin{array}{l}\mathrm{Ac})_{2} \cdot \mathrm{H}_{2} \mathrm{O} \\
\mathrm{mol} \%)\end{array}$ & temp. $\left({ }^{\circ} \mathrm{C}\right)$ & solvent & $\begin{array}{l}\text { NMR yield } \\
\left(\%, \mathbf{3 a}: \mathbf{3} \mathbf{a}^{\prime}\right)\end{array}$ \\
\hline 1 & 5 & 5 & & 50 & THF & $74(99: 1)$ \\
\hline 2 & 10 & 0 & & 50 & THF & $52(99: 1)$ \\
\hline 3 & 0 & 10 & & 50 & THF & $30(99: 1)$ \\
\hline 4 & 5 & 5 & & $\mathrm{rt}$ & THF & $30(99: 1)$ \\
\hline 5 & 5 & 5 & & 70 & THF & $58(99: 1)$ \\
\hline 6 & 5 & 5 & & 50 & toluene & $48(99: 1)$ \\
\hline 7 & 5 & 5 & & 50 & 1,4-dioxane & $\begin{array}{l}82(99: 1) \\
{[80(99: 1)]^{b}}\end{array}$ \\
\hline 8 & 5 & 5 & & 50 & DMF & $11(83: 17)$ \\
\hline 9 & 5 & 5 & & 50 & $\mathrm{MeCN}$ & ND \\
\hline 10 & 5 & 5 & & 50 & $\mathrm{MeOH}$ & $24(99: 1)$ \\
\hline
\end{tabular}

${ }^{a}$ Reaction conditions: 1 (1.0 mmol), benzyl azide (1.2 mmol), Cul (x mol \%), $\mathrm{Cu}(\mathrm{OAc})_{2} \cdot \mathrm{H}_{2} \mathrm{O}$ (y mol \%) in 1,4-dioxane at temp. $\left({ }^{\circ} \mathrm{C}\right.$ ) for $20 \mathrm{~h} .{ }^{b} \mathrm{An}$ isolated yield. 


\section{Control Experiment with Triisopropylsilyl (TIPS)-protected Bromoethyne 4}

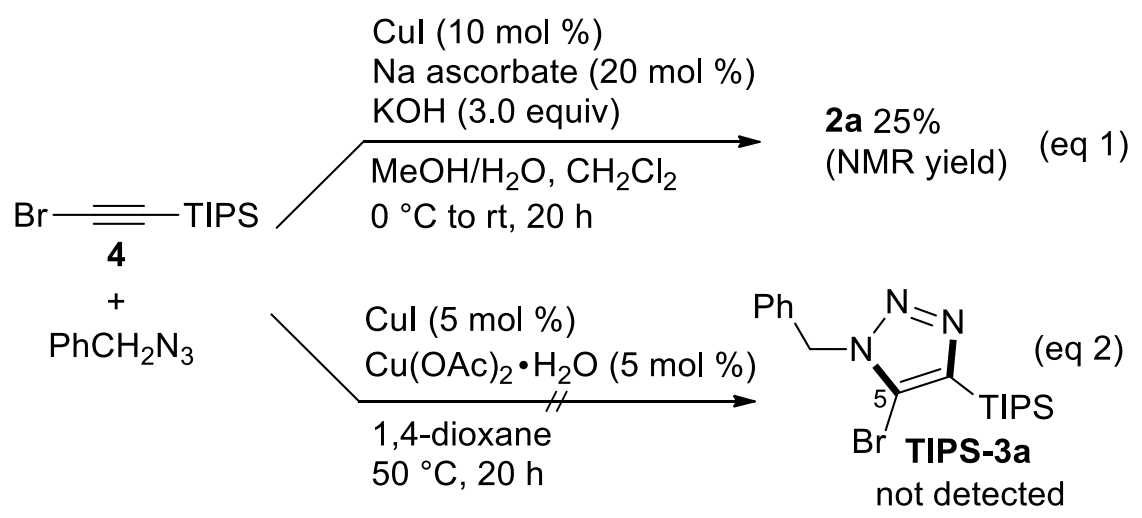

Scheme S1. Attempted regioselective syntheses of 4- and 5-bromo-substituted 1,2,3-triazoles from $\mathrm{Br}-\mathrm{C} \equiv \mathrm{C}-\mathrm{TIPS} 4$.

We postulated triisopropylsilyl (TIPS)-protected bromoethyne $\mathbf{4}$ may be utilized as one starting material in this regiodivergent $\mathrm{CuAAC}$. Under the standard condition of dephosphorylative (herein desilylative) CuAAC, the corresponding 4-bromotriazole 2a was only detected in 25\% NMR yield (eq 1). We also investigated further improvement of this desilylative CuAAC, but no satisfactory result was obtained. Continuously, direct CuAAC with $\mathbf{4}$ under standard condition of dual copper catalysis was conducted, but no product was obtained (recovery of starting materials) (eq 2). 


\section{Crystallographic Data}

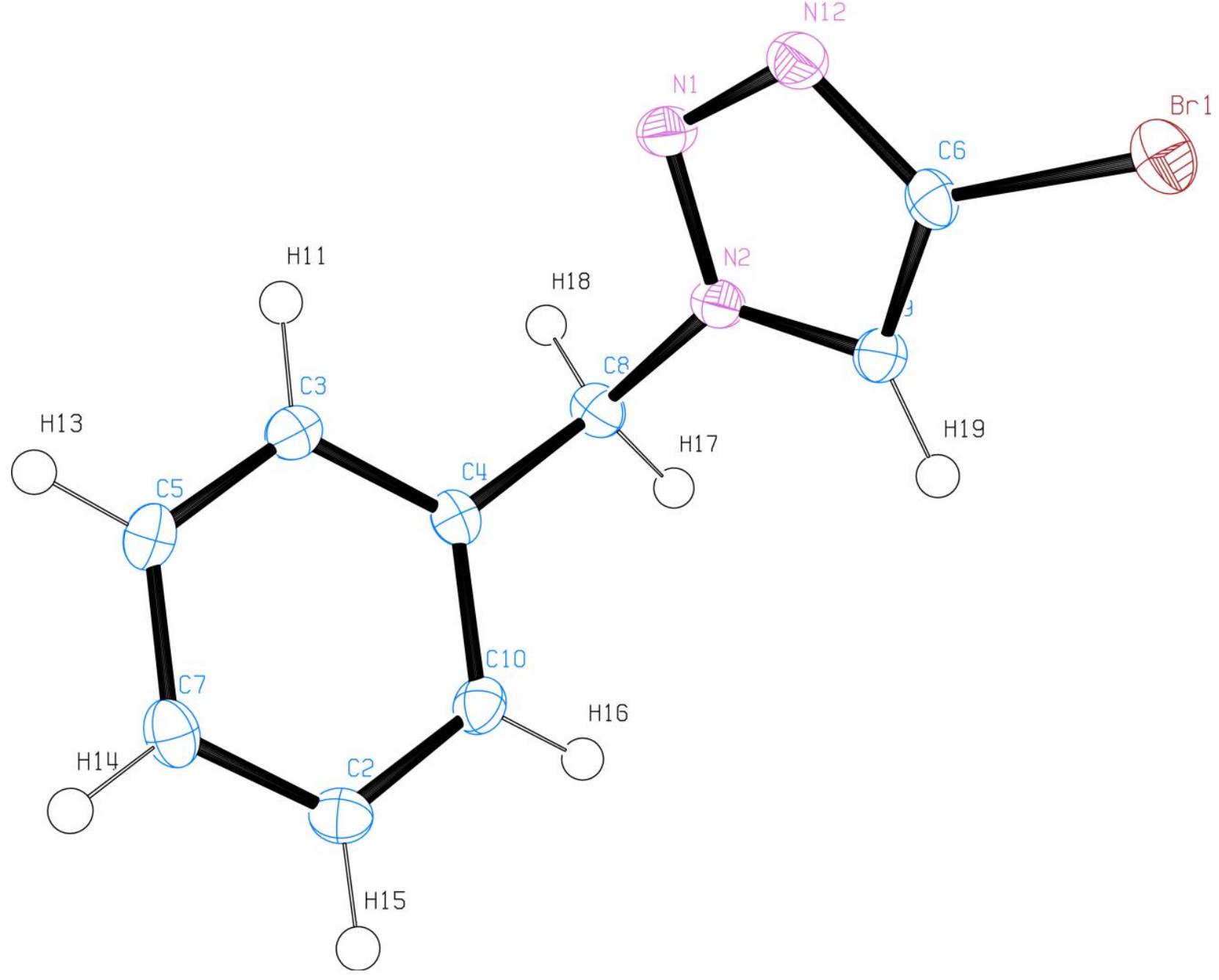

Figure S1. ORTEP drawing of $\mathbf{2 a}$ at 50\% ellipsoid probability (CCDC 2002508).

Single crystals of $\mathbf{2 a}$ were obtained by slow evaporation of solution of $10 \mathrm{mg}$ of $\mathbf{2 a}$ in $1 \mathrm{~mL}$ of chloroform. 
Table S3. Crystal data and structure refinement details for compound 2a.

Empirical Formula

Formula Weight

Crystal Color, Habit

Crystal Dimensions

Crystal System

Lattice Type

Lattice Parameters

Space Group

$Z$ value

$\mathrm{D}_{\text {calc }}$

$\mathrm{F}_{000}$

$\mu(\operatorname{MoK} \alpha)$

Diffractometer

Radiation

Voltage, Current

Temperature

No. of Reflections Measured

Residuals: R1 (I>2.00б(I))

Residuals: R (All reflections)

Residuals: wR2 (All reflections)

Goodness of Fit Indicator

\section{$\mathrm{C}_{9} \mathrm{H}_{8} \mathrm{BrN}_{3}$}

238.09

unknown, platelet

$0.100 \times 0.030 \times 0.030 \mathrm{~mm}$

monoclinic

Primitive

$a=9.364(4) \AA$

$\mathrm{b}=5.656(2) \AA$

$\mathrm{c}=17.689(6) \AA$

$\beta=100.026(5)^{\circ}$

$\mathrm{V}=922.6(6) \AA^{3}$

$\mathrm{P} 2{ }_{1} / \mathrm{c}$ (\#14)

4

$1.714 \mathrm{~g} / \mathrm{cm}^{3}$

472.00

$44.213 \mathrm{~cm}^{-1}$

Saturn70

$\operatorname{MoK} \alpha(\lambda=0.71075 \AA)$

$50 \mathrm{kV}, 24 \mathrm{~mA}$

$0.0{ }^{\circ} \mathrm{C}$

Total: 13922

Unique: $2108\left(\mathrm{R}_{\text {int }}=0.0369\right)$

0.0260

0.0311

0.0618

1.065 


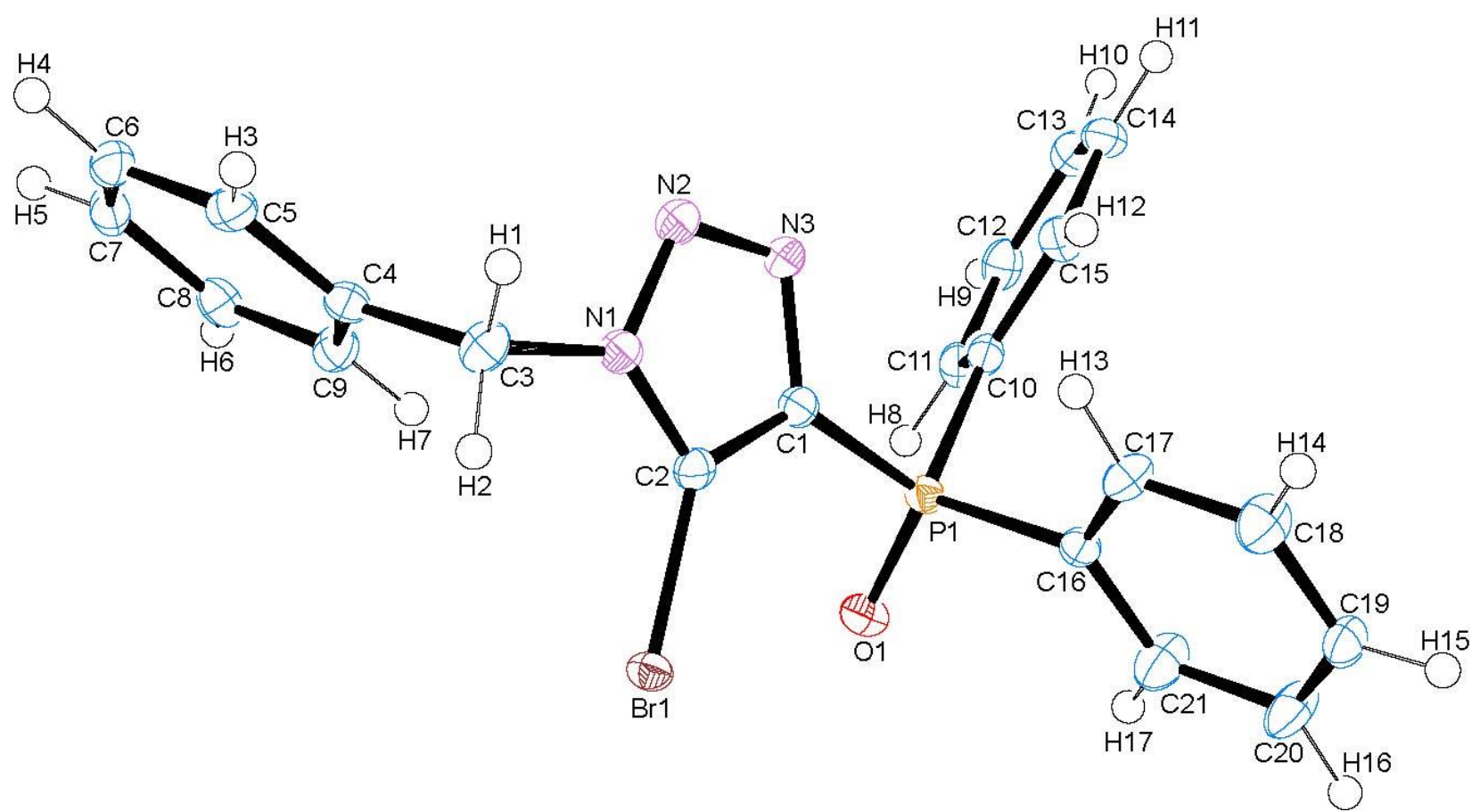

Figure S2. ORTEP drawing of 3a at 50\% ellipsoid probability (CCDC 2002595).

Single crystals of $\mathbf{3 a}$ were obtained by slow evaporation of solution of $10 \mathrm{mg}$ of $\mathbf{3 a}$ in $1 \mathrm{~mL}$ of chloroform. 
Table S4. Crystal data and structure refinement details for compound 3a.

\begin{tabular}{|c|c|}
\hline Empirical Formula & $\mathrm{C}_{21} \mathrm{H}_{17} \mathrm{BrN}_{3} \mathrm{OP}$ \\
\hline Formula Weight & 438.26 \\
\hline Crystal Color, Habit & colorless, block \\
\hline Crystal Dimensions & $0.376 \times 0.127 \times 0.080 \mathrm{~mm}$ \\
\hline Crystal System & monoclinic \\
\hline Lattice Type & Primitive \\
\hline \multirow[t]{5}{*}{ Lattice Parameters } & $\mathrm{a}=11.992(3) \AA$ \\
\hline & $\mathrm{b}=6.4480(18) \AA$ \\
\hline & $c=24.891(7) \AA$ \\
\hline & $\beta=102.981(3)^{\circ}$ \\
\hline & $\mathrm{V}=1875.5(9) \AA^{3}$ \\
\hline Space Group & $\mathrm{P} 2{ }_{1} / \mathrm{c}(\# 14)$ \\
\hline $\mathrm{Z}$ value & 4 \\
\hline $\mathrm{D}_{\text {calc }}$ & $1.552 \mathrm{~g} / \mathrm{cm}^{3}$ \\
\hline $\mathrm{F}_{000}$ & 888.00 \\
\hline$\mu(\operatorname{MoK} \alpha)$ & $22.998 \mathrm{~cm}^{-1}$ \\
\hline Diffractometer & Saturn70 \\
\hline Radiation & $\operatorname{MoK} \alpha(\lambda=0.71075 \AA)$ \\
\hline Voltage, Current & $50 \mathrm{kV}, 24 \mathrm{~mA}$ \\
\hline Temperature & $0.0^{\circ} \mathrm{C}$ \\
\hline \multirow[t]{2}{*}{ No. of Reflections Measured } & Total: 25259 \\
\hline & Unique: $3643\left(\mathrm{R}_{\text {int }}=0.0921\right)$ \\
\hline Residuals: R1 (I>2.00б(I)) & 0.0300 \\
\hline Residuals: R (All reflections) & 0.0341 \\
\hline Residuals: wR2 (All reflections) & 0.0777 \\
\hline Goodness of Fit Indicator & 1.077 \\
\hline
\end{tabular}




\section{References and Notes}

(1) Fulmer, G. R.; Miller, A. J. M.; Sherden, N. H.; Gottlieb, H. E.; Nudelman, A. ; Stoltz, B. M.; Bercaw, J. E.; Goldberg, K. I. NMR Chemical Shifts of Trace Impurities: Common Laboratory Solvents, Organics, and Gases in Deuterated Solvents Relevant to the Organometallic Chemist. Organometallics 2010, 29, 2176-2179.

(2) Dinoiu, V.; Lü, J.-M. Synthesis of new trifluoromethyl-containing 1-(3,5-dialkyl-4hydroxybenzyl)-pyrazole and -pyrazol-5-one deriatives and their corresponding aroxyls. J. Serb. Chem. Soc. 2006, 71, 323-330. doi: 10.2298/JSC0604323D.

(3) Keglevich, G.; Bálint, E.; Kangyal, R.; Bálint, M.; Milen, M. A Critical Overview of the Kabachnik-Fields Reactions Utilizing Trialkyl Phosphites in Water as the Reaction Medium: A Study of the Benzaldehyde-Benzylamine Triethyl Phosphite/Diethyl Phosphite Models. Heteroatom Chem. 2014, 25, 282-289. doi: 10.1002/hc.21192.

(4) Pflugrath, J. W. The finer things in X-ray diffraction data collection. Acta Crystallogr., Sect. D: Biol. Crystallogr. 1999, 55, 1718-1725. doi: 10.1107/S090744499900935X

(5) Burla, M. C.; Caliandro, R.; Camalli, M.; Carrozzini, B.; Cascarano, G. L.; De Caro, L.; Giacovazzo, C.; Polidori, G.; Spagna, R. SIR2004: an improved tool for crystal structure determination and refinement. J. Appl. Crystallogr. 2005, 38, 381-388.

doi: 10.1107/S002188980403225X

(6) Sheldrick, G. M. A short history of SHELX. Acta Crystallogr. A 2008, 64, 112-122. doi: 10.1107/S0108767307043930.

(7) (a) Padovan, P.; Tartaggia, S.; Lorenzon, S.; Rosso, E.; Zonta, C.; De Lucchi, O.; Fabris, F. 1Bromo-2-(diphenylphosphinoyl)ethyne and 1-bromo-2-(p-tolylsulfinyl)ethyne: versatile reagents eventually leading to benzocyclotrimers. Tetrahedron Lett. 2009, 50, 1973-1976. (b) Perez, V.; Fadel, A.; Rabasso, N. Synthesis of $N$-Sulfonyl Ynamido-Phosphonates: Valuable Partners for Cycloadditions. Synthesis 2017, 49, 4035-4044. (c) Okuda, Y.; Seo, T.; Shigezane, Y.; Watanabe, H.; Akashi, H.; Iwanaga, T.; Orita, A. Synthesis of $\mathrm{Ph}_{2} \mathrm{P}(\mathrm{O})$-Stabilized Ynamines via C(sp)-N Bond Formation and Their Dephosphorylative Copper-Catalyzed Click Reaction. Chem. Lett. 2019, $48,1484-1487$.

(8) Asano, K.; Matsubara, S. Effects of a Flexible Alkyl Chain on a Ligand for CuAAC Reaction. Org. Lett. 2010, 12, 4988-4991. 
(9) Hansen, S. G.; Jensen. H. H. Microwave Irradiation as an Effective Means of Synthesizing Unsubstituted $N$-Linked 1,2,3-Triazoles from Vinyl Acetate and Azides. Synlett 2009, 3275-3278. (10) Büll, C.; Heise, T.; van Hilten, N.; Pijnenborg, J. F. A.; Bloemendal, V. R. L. J.; Gerrits, L.; Kers-Rebel, E. D.; Ritschel, T.; den Brok, M. H.; Adema, G. J.; Boltje, T. J. Steering Siglec-Sialic Acid Interactions on Living Cells using Bioorthogonal Chemistry. Angew. Chem. Int. Ed. 2017, 56, 3309-3313.

(11) Suzuki, T.; Ota, Y.; Kasuya, Y.; Mutsuga, M.; Kawamura, Y.; Tsumoto, H.; Nakagawa, H.; Finn, M. G.; Miyata, N. An Unexpected Example of Protein-Templated Click Chemistry. Angew. Chem. Int. Ed. 2010, 49, 6817-6820.

(12) Stefely, J. A.; Palchaudhuri, R.; Miller, P. A.; Peterson, R. J.; Moraski, G. J.; Hergenrother, P. J.; Miller, M. J. $N$-((1-Benzyl-1H-1,2,3-triazol-4-yl)methyl)arylamide as a New Scaffold that Provides Rapid Access to Antimicrotubule Agents: Synthesis and Evaluation of Antiproliferative Activity Against Select Cancer Cell Lines. J. Med. Chem. 2010, 53, 3389-3395.

(13) Li, J.; Cao, J.-J.; Wei, J.-F.; Shi, X.-Y.; Zhang, L.-H.; Feng, J.-J.; Chen, Z.-G. Ionic Liquid Brush as a Highly Efficient and Reusable Catalyst for On-Water Nucleophilic Substitutions. Eur. J. Org. Chem. 2011, 229-233.

(14) Shu, C.; Wang, Y.-H.; Zhou, B.; Li, X.-L.; Ping, Y.-F.; Lu, X.; Ye, L.-W. Generation of $\alpha-$ Imino Gold Carbenes through Gold-Catalyzed Intermolecular Reaction of Azides with Ynamides. J. Am. Chem. Soc. 2015, 137, 9567-9570.

(15) Liu, M.; Reiser, O. A Copper(I) Isonitrile Complex as a Heterogeneous Catalyst for Azide-Alkyne Cycloaddition in Water. Org. Lett. 2011, 13, 1102-1105.

(16) Büll, C.; Heise, T,; Beurskens, D. M. H.; Riemersma, M.; Ashikov, A.; Rutjes, F. P. J. T.; van Kuppevelt, T. H.; Lefeber, D. J.; den Brok, M. H.; Adema, G. J.; Boltje, T. J. Sialic Acid Glycoengineering Using an Unnatural Sialic Acid for the Detection of Sialoglycan Biosynthesis Defects and On-Cell Synthesis of Siglec Ligands. ACS Chem. Biol. 2015, 10, 2353-2363.

(17) Hu, M.; Li, J.; Yao, S. Q. In Situ "Click” Assembly of Small Molecule Matrix Metalloprotease Inhibitors Containing Zinc-Chelating Groups. Org. Lett. 2008, 10, 5529-5531.

(18) Zhua, W.; Ma, D. Synthesis of aryl azides and vinyl azides via proline-promoted CuI-catalyzed coupling reactions. Chem. Commun. 2004, 888-889. 
(19) Begtrup, M. Reactions between Azolium Salts and Nucleophilic Reagents. II. Bromo-1,2,3triazolium Salts and Sodium Hydroxide. Acta Chem. Scand. 1971, 25, 249-259. doi: 10.3891/acta.chem.scand.25-0249.

(20) Jiang, Y.; Que, L.; Xu, J.; Qin, D.; Cai, T. (2016) Preparation method for 1-substituted-1H1,2,3-triazole-4-carboxylic acid. WO2016062175.]

(21) Li, L.; Shang, T.; Ma, X.; Guo, H.; Zhu, A.; Zhang, G. 4-Trimethylsilyl-5-iodo-1,2,3-triazole: A Key Precursor for the Divergent Syntheses of 1,5-Disubstituted 1,2,3-Triazoles. Synlett, 2015, 26, 695-699.

(22) Suzuki, T.; Ota, Y.; Ri, M.; Bando, M.; GotohA.; Itoh, Y.; Tsumoto, H.; Tatum, P. R.; Mizukami, T.; Nakagawa, H.; Iida, S.; Ueda, R.; Shirahige, K.; Miyata, N. Rapid Discovery of Highly Potent and Se-lective Inhibitors of Histone Deacetylase 8 Using Click Chemistry to Generate Candidate Libraries J. Med. Chem. 2012, 55, 9562-9575. 


\section{Copies of ${ }^{1} \mathbf{H},{ }^{13} \mathrm{C}\left\{{ }^{1} \mathrm{H}\right\},{ }^{19} \mathrm{~F}\left\{{ }^{1} \mathrm{H}\right\}$ and ${ }^{31} \mathbf{P}\left\{{ }^{1} \mathrm{H}\right\}$ NMR Charts}

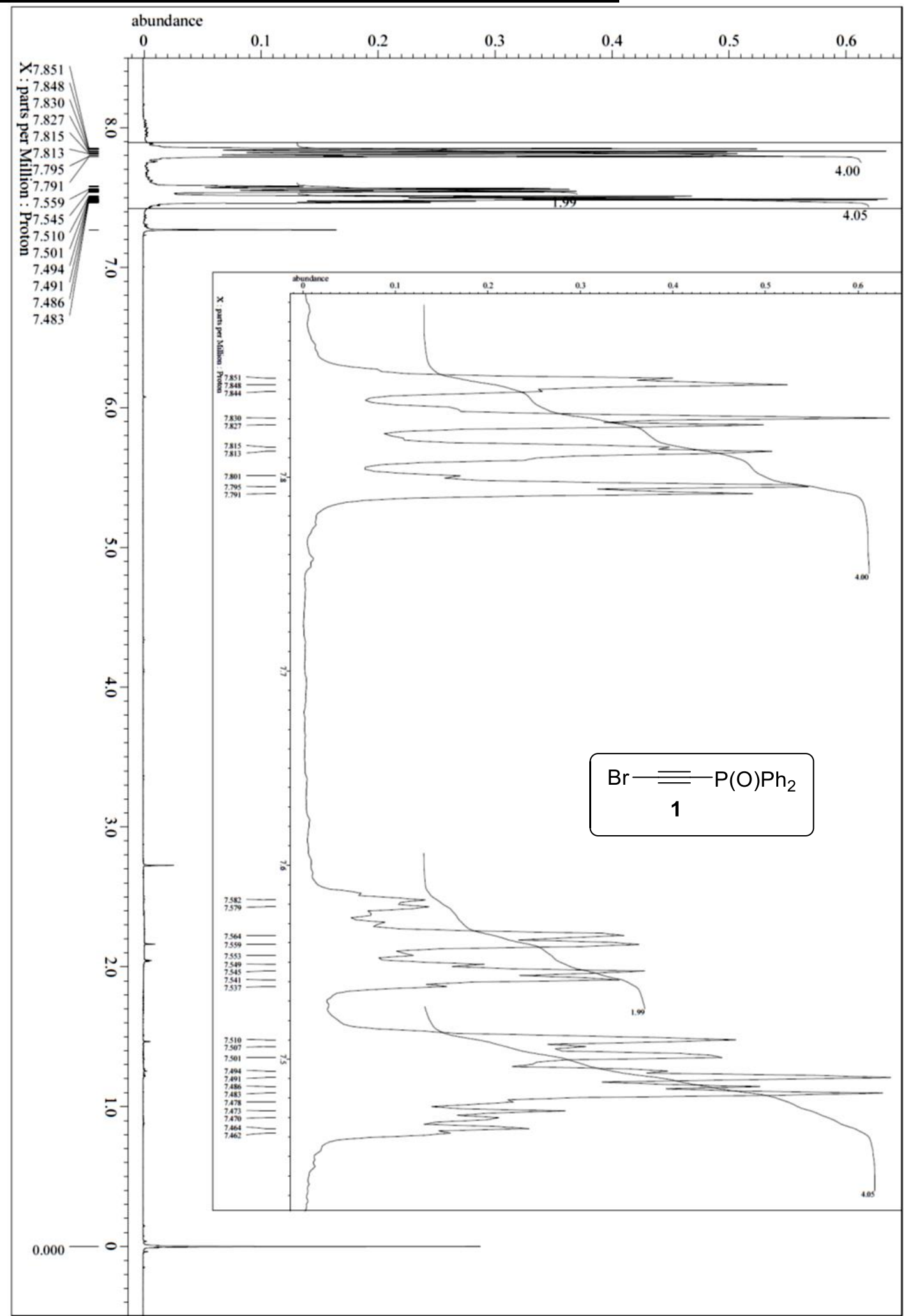

Figure S3. ${ }^{1} \mathrm{H}$ NMR (400 MHz) spectrum of $\mathbf{1}\left(\mathrm{CDCl}_{3}, \mathrm{rt}\right)$ 


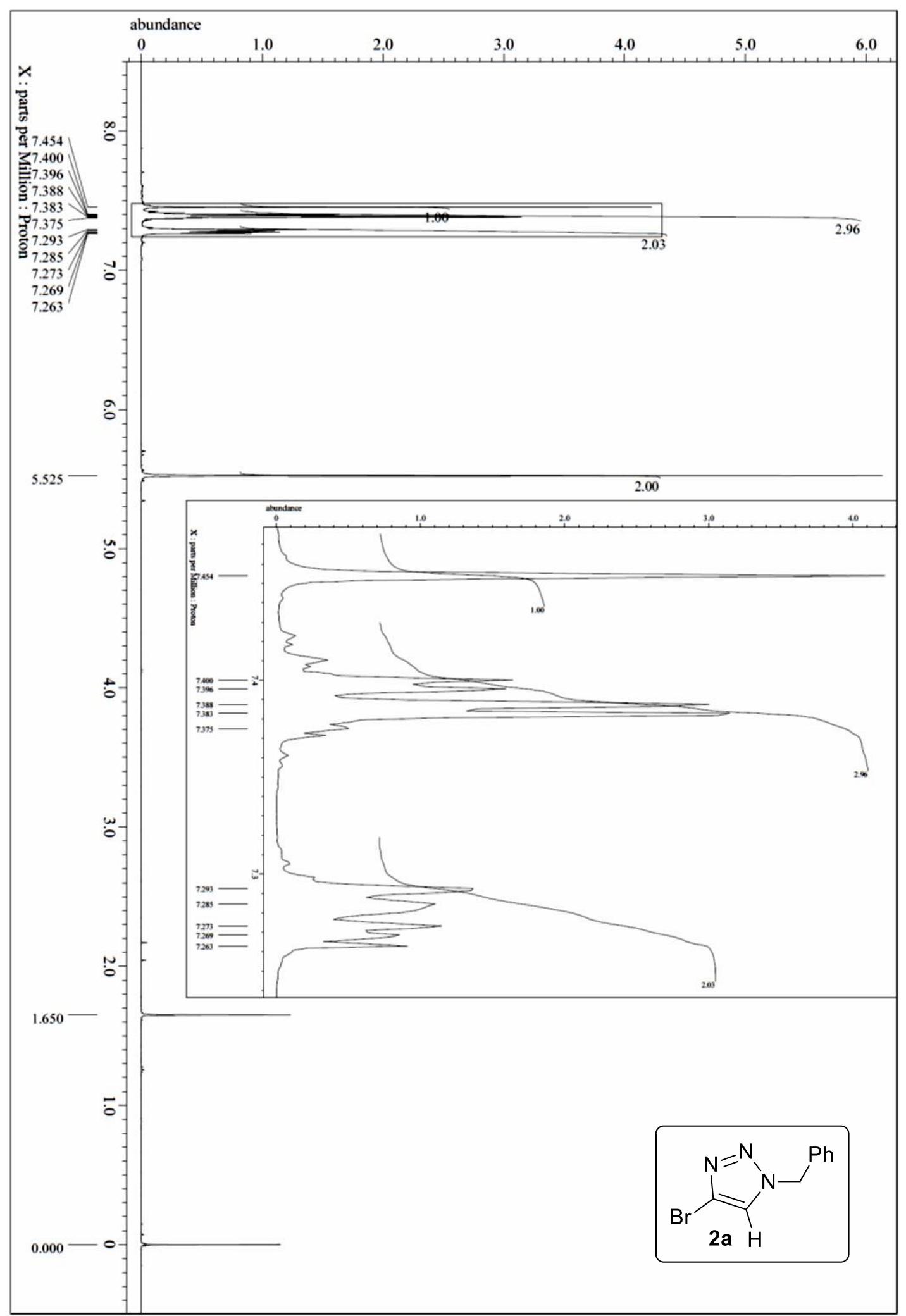




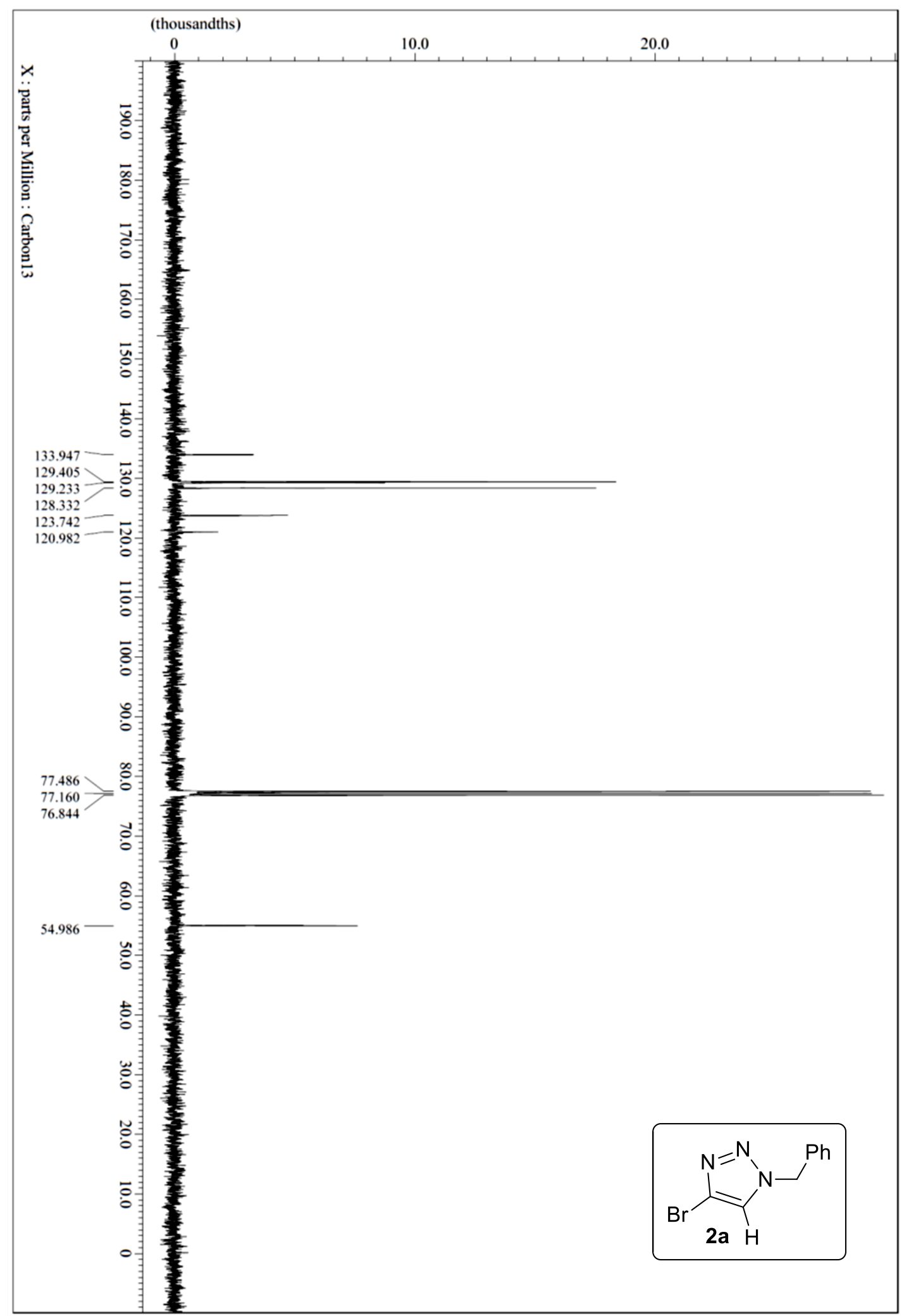

Figure S4. ${ }^{1} \mathrm{H}(400 \mathrm{MHz})$ and ${ }^{13} \mathrm{C}\left\{{ }^{1} \mathrm{H}\right\} \mathrm{NMR}(101 \mathrm{MHz})$ spectra of $\mathbf{2 a}\left(\mathrm{CDCl}_{3}, \mathrm{rt}\right)$ 


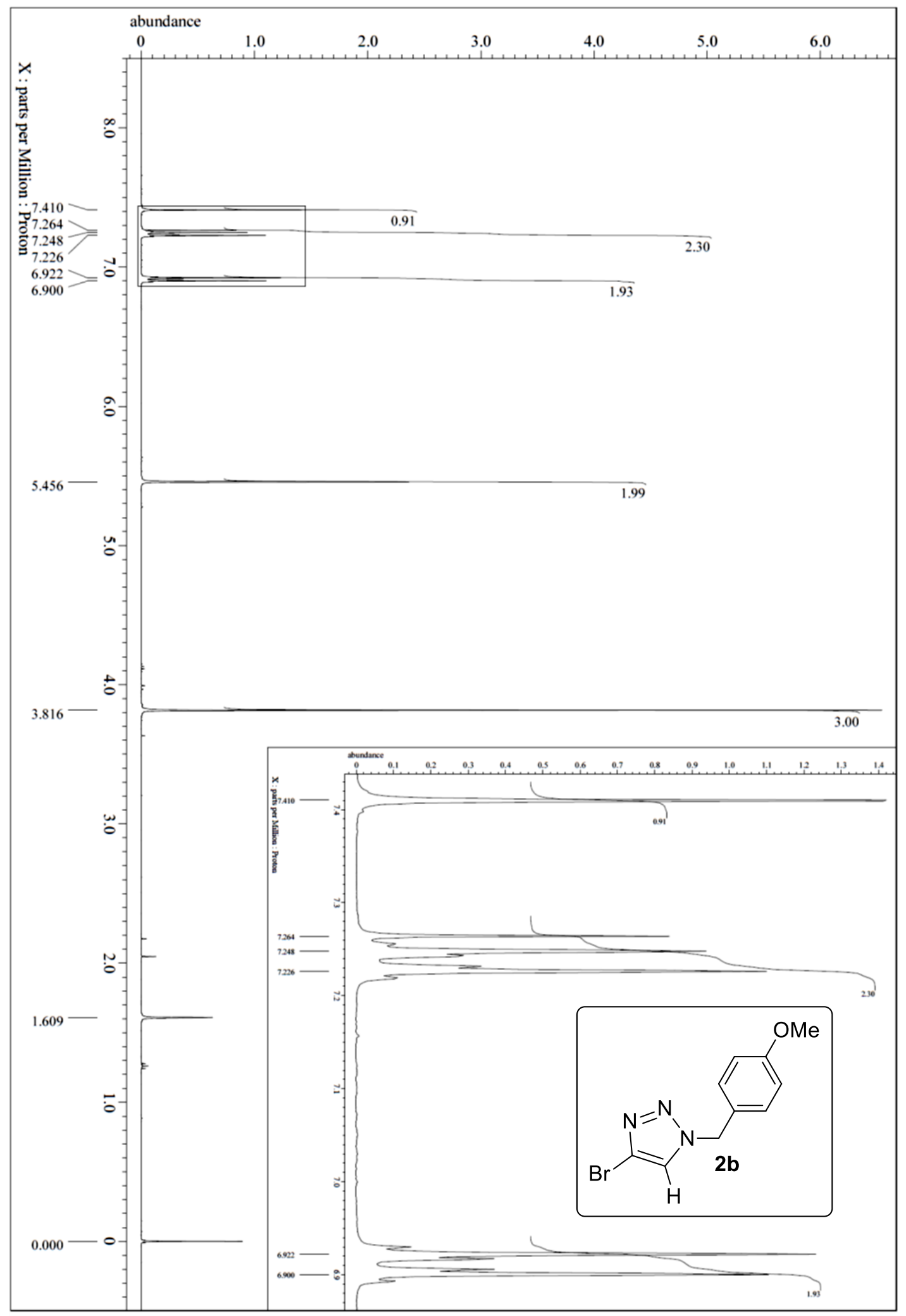




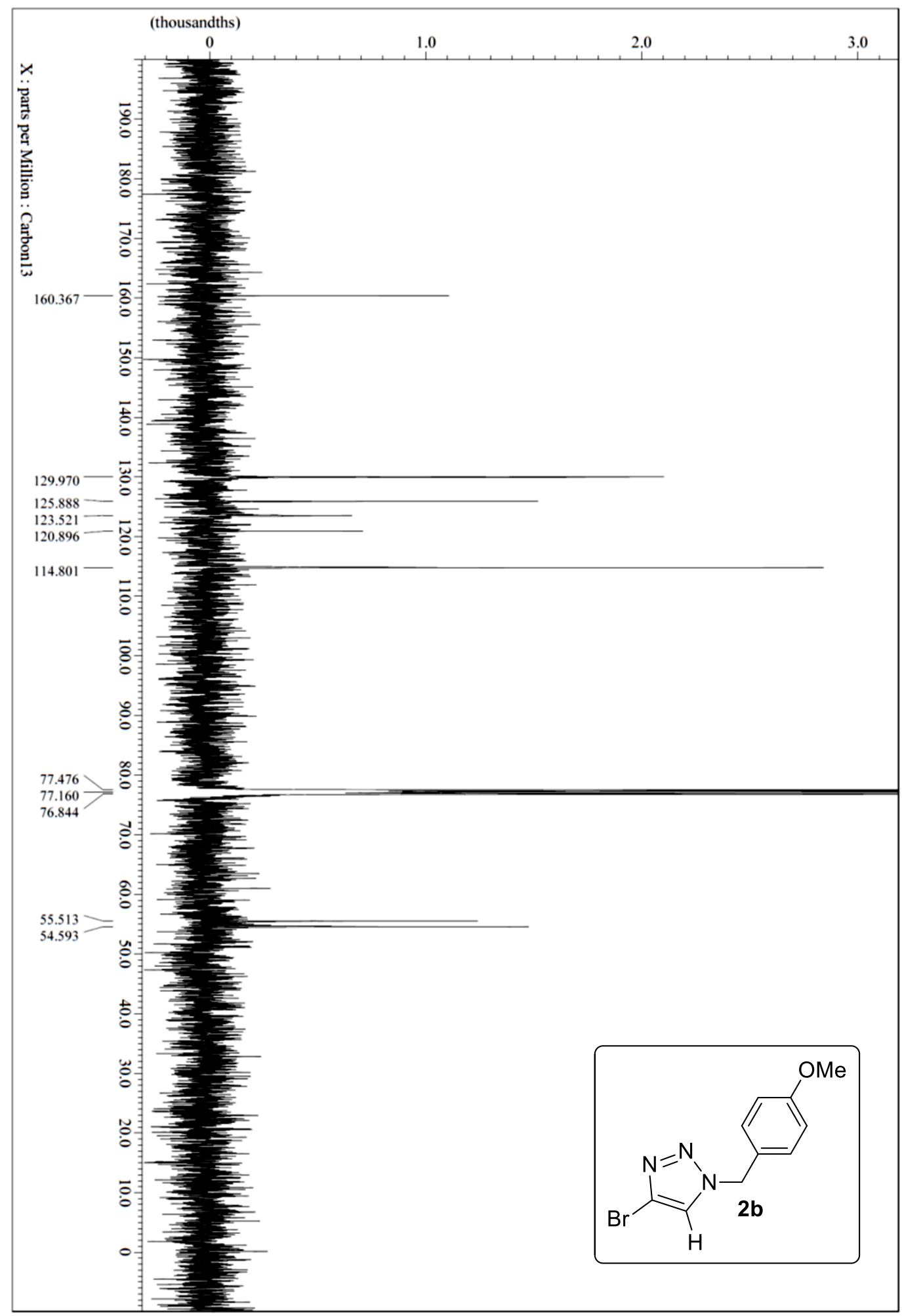

Figure S5. ${ }^{1} \mathrm{H}(400 \mathrm{MHz})$ and ${ }^{13} \mathrm{C}\left\{{ }^{1} \mathrm{H}\right\}$ NMR $(101 \mathrm{MHz})$ spectra of $\mathbf{2 b}\left(\mathrm{CDCl}_{3}, \mathrm{rt}\right)$ 


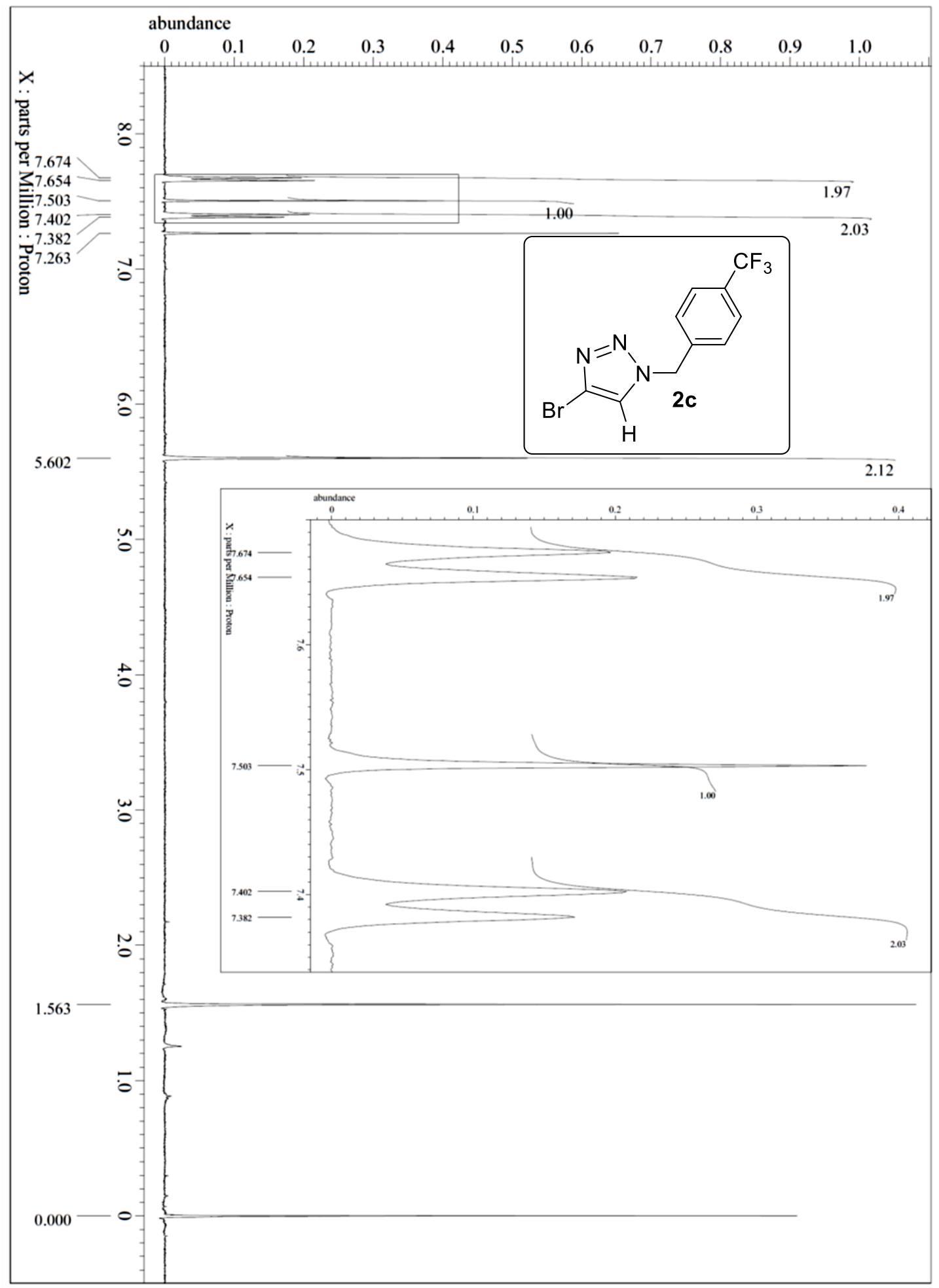




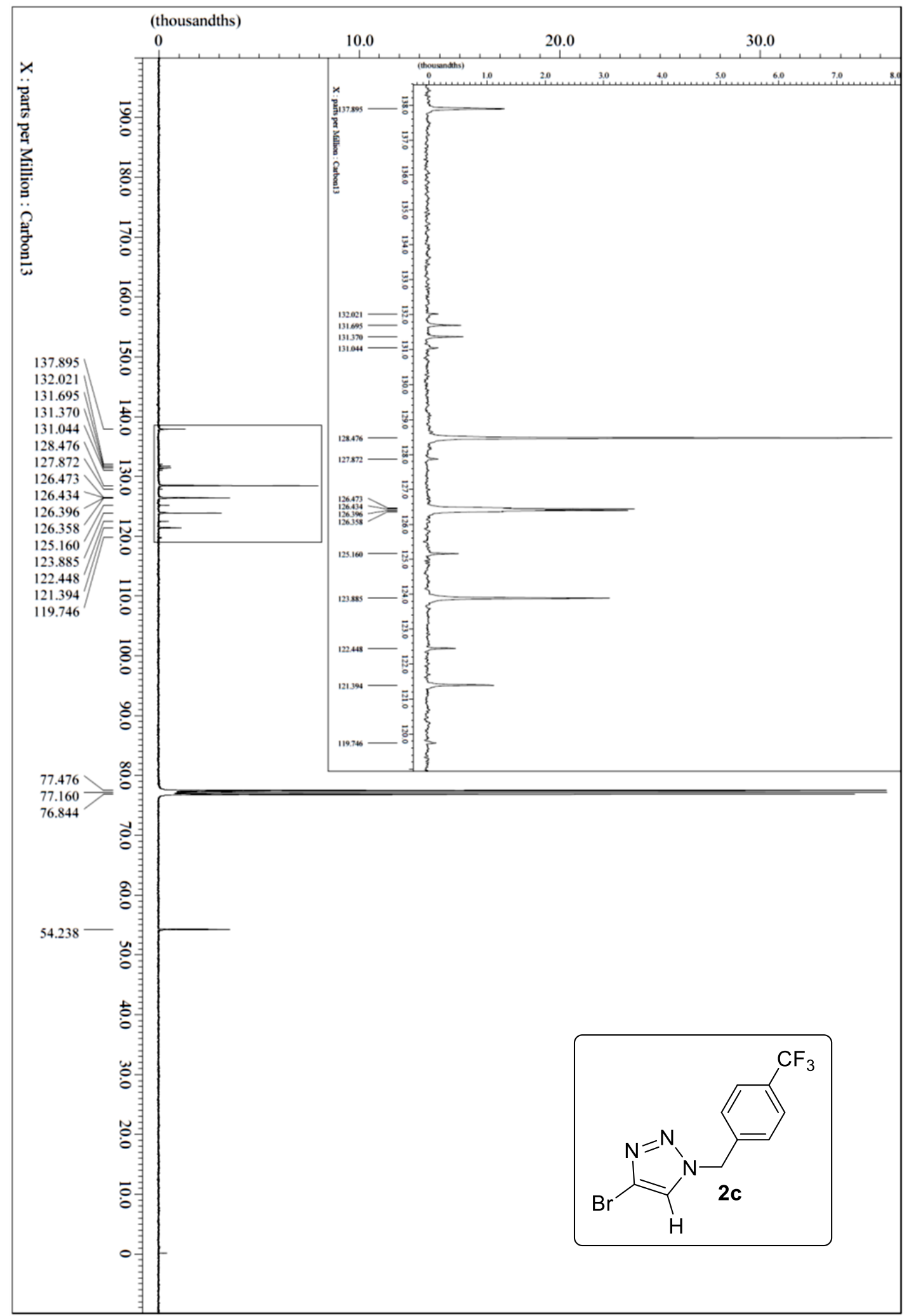




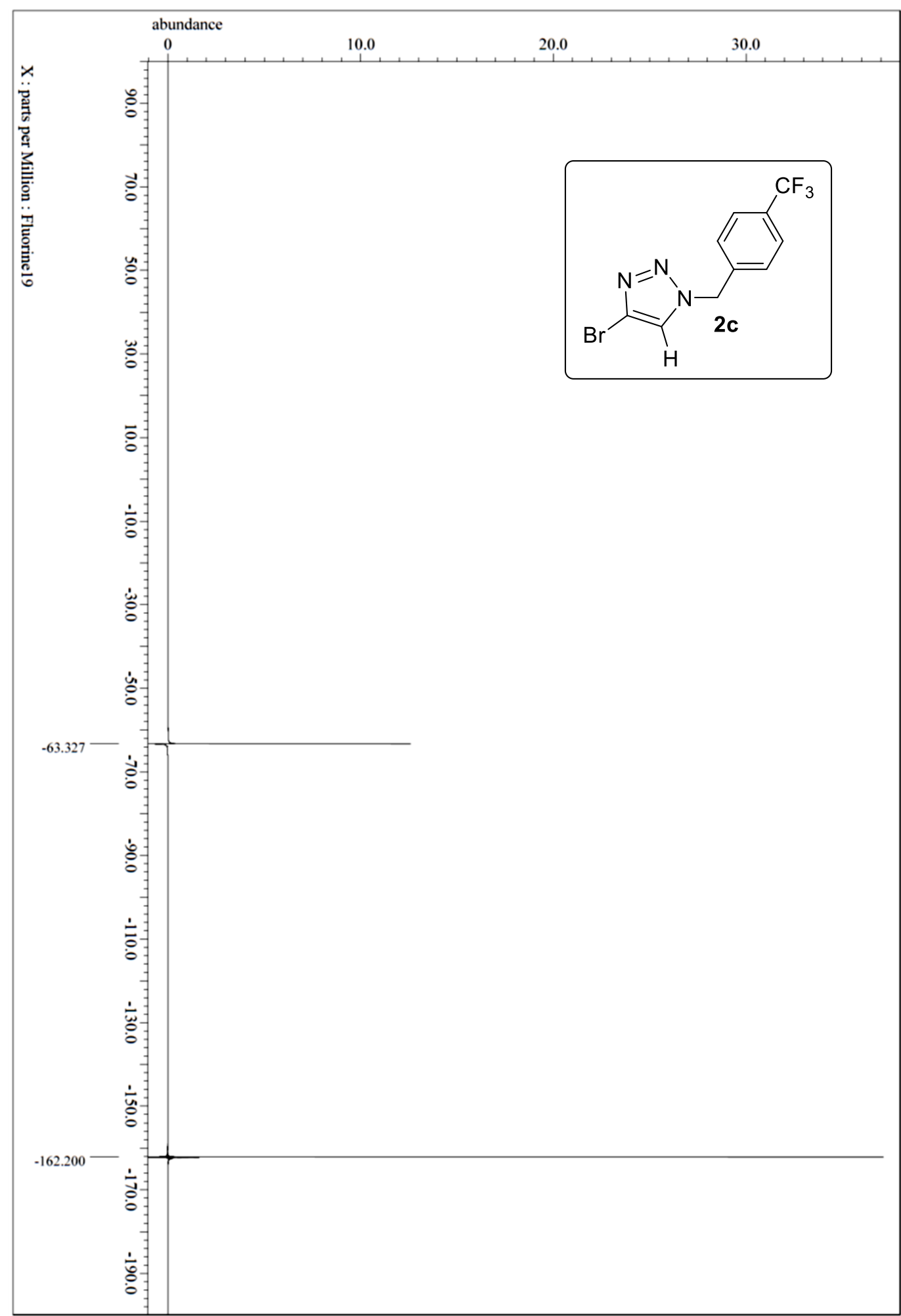

Figure S6. ${ }^{1} \mathrm{H}(400 \mathrm{MHz}),{ }^{13} \mathrm{C}\left\{{ }^{1} \mathrm{H}\right\} \mathrm{NMR}(101 \mathrm{MHz})$ and ${ }^{19} \mathrm{~F}\left\{{ }^{1} \mathrm{H}\right\}(376 \mathrm{MHz})$ spectra of 2c $\left(\mathrm{CDCl}_{3}, \mathrm{rt}\right)$ 


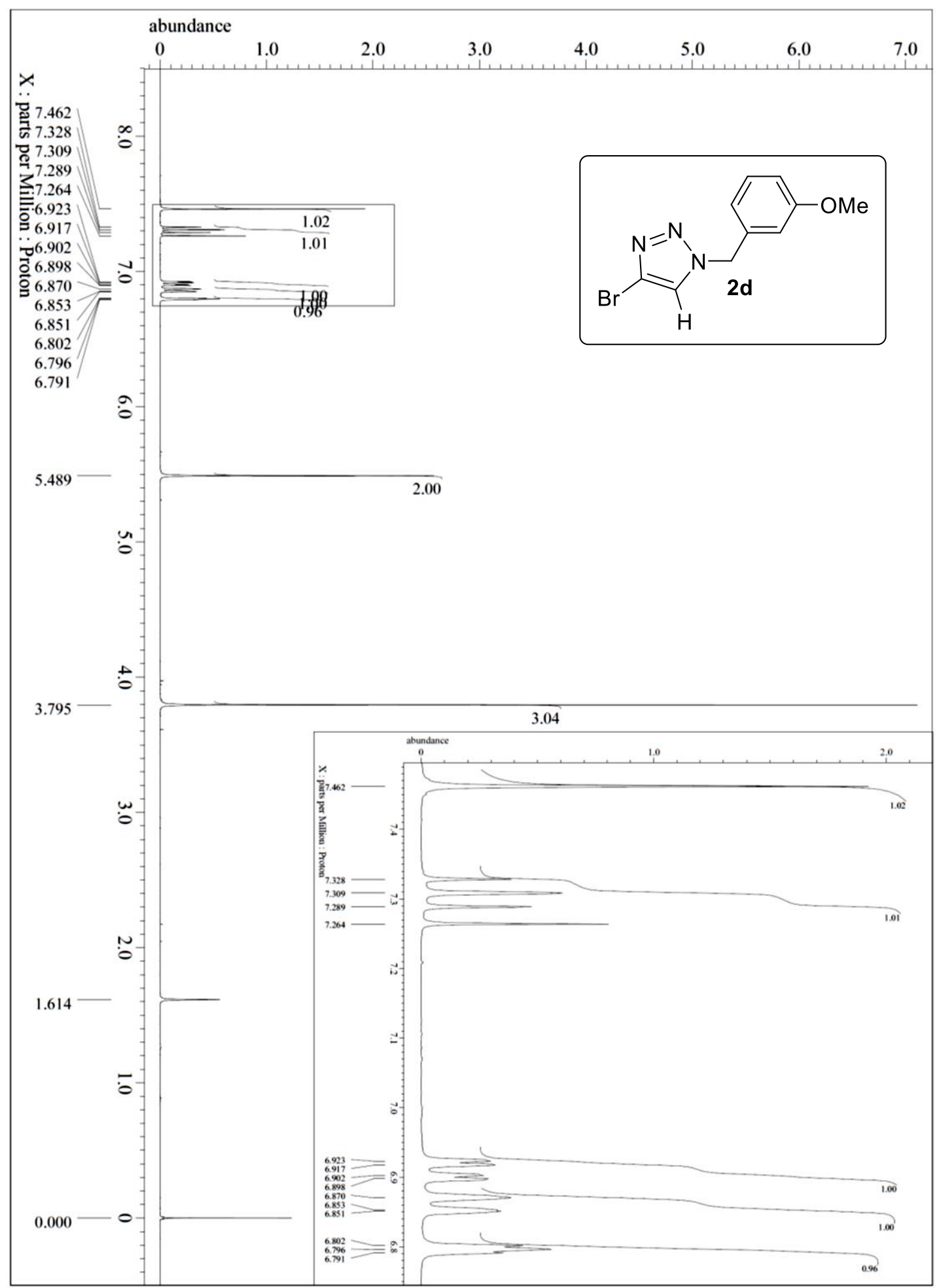




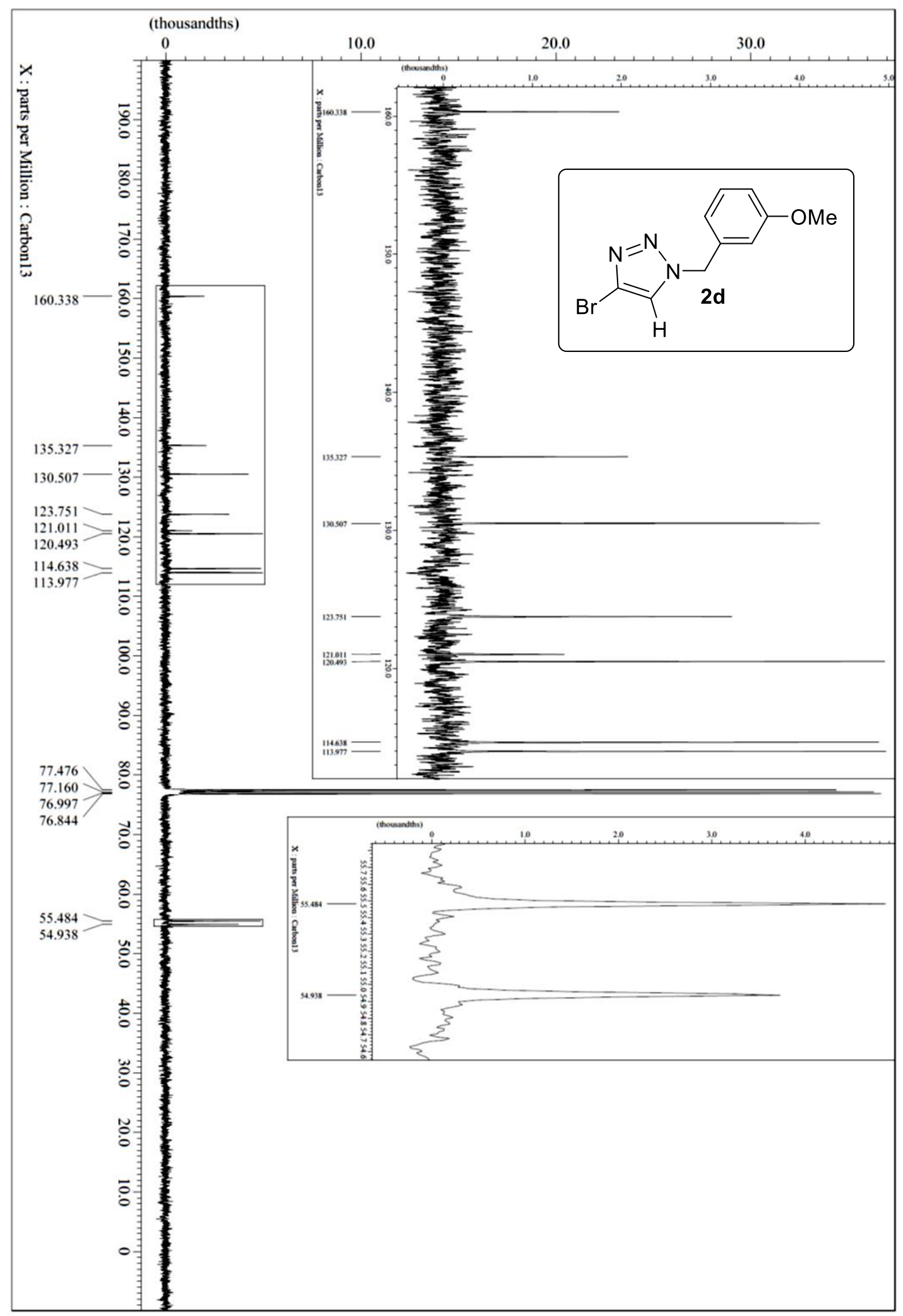

Figure S7. ${ }^{1} \mathrm{H}(400 \mathrm{MHz})$ and ${ }^{13} \mathrm{C}\left\{{ }^{1} \mathrm{H}\right\} \mathrm{NMR}(101 \mathrm{MHz})$ spectra of $2 \mathbf{d}\left(\mathrm{CDCl}_{3}, \mathrm{rt}\right)$ 


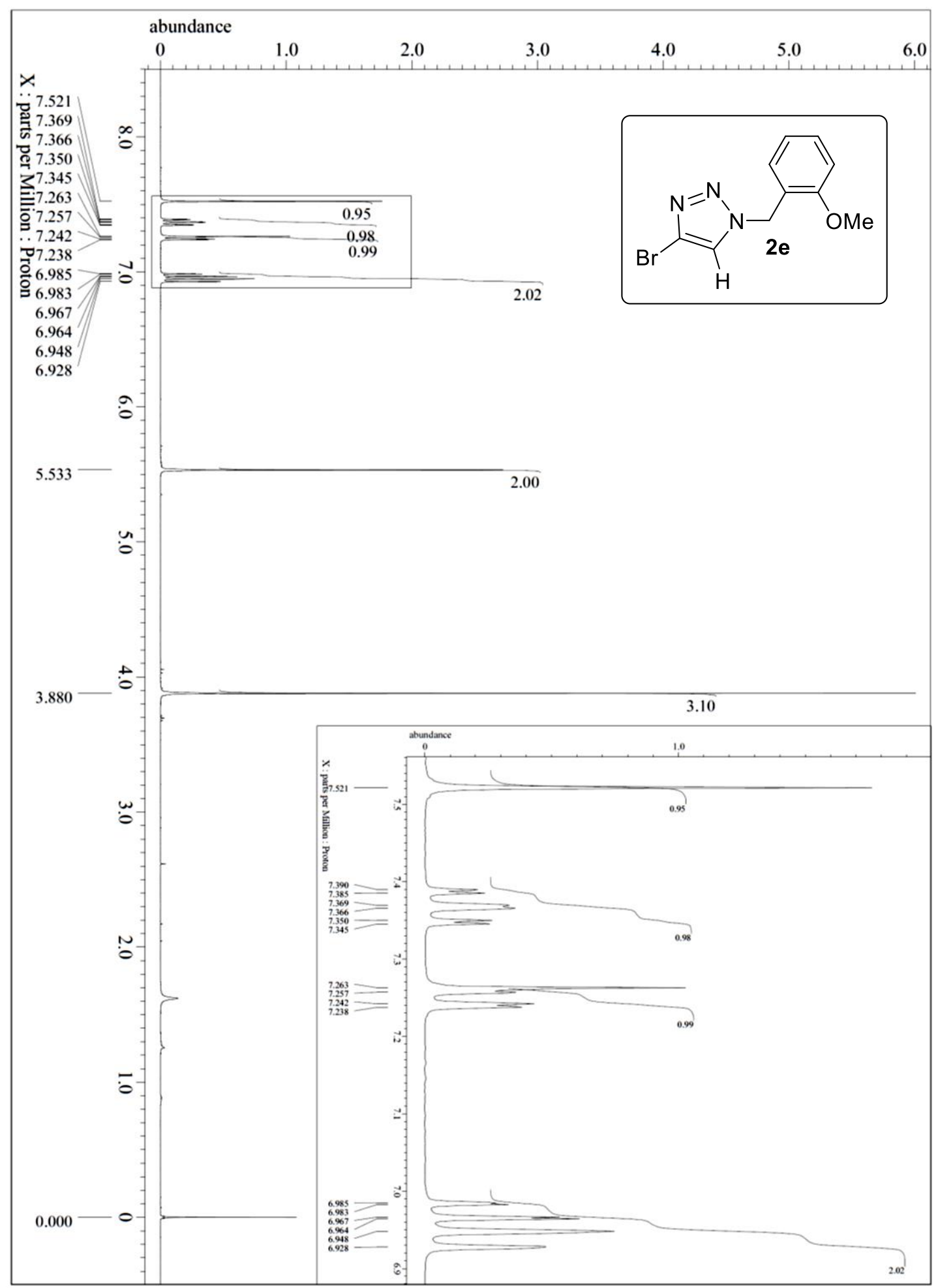




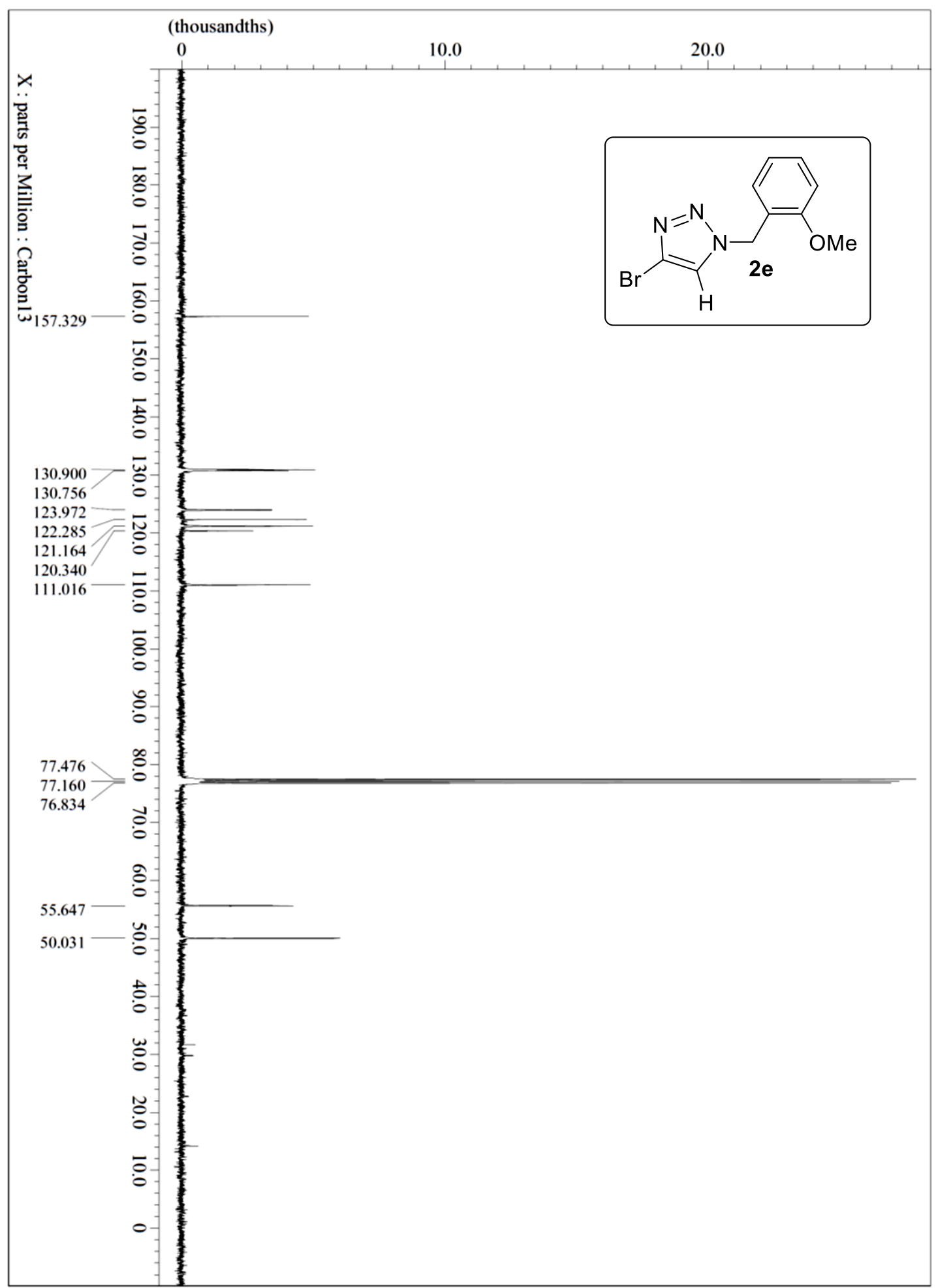

Figure S8. ${ }^{1} \mathrm{H}(400 \mathrm{MHz})$ and ${ }^{13} \mathrm{C}\left\{{ }^{1} \mathrm{H}\right\} \mathrm{NMR}(101 \mathrm{MHz})$ spectra of $\mathbf{2 e}\left(\mathrm{CDCl}_{3}, \mathrm{rt}\right)$ 


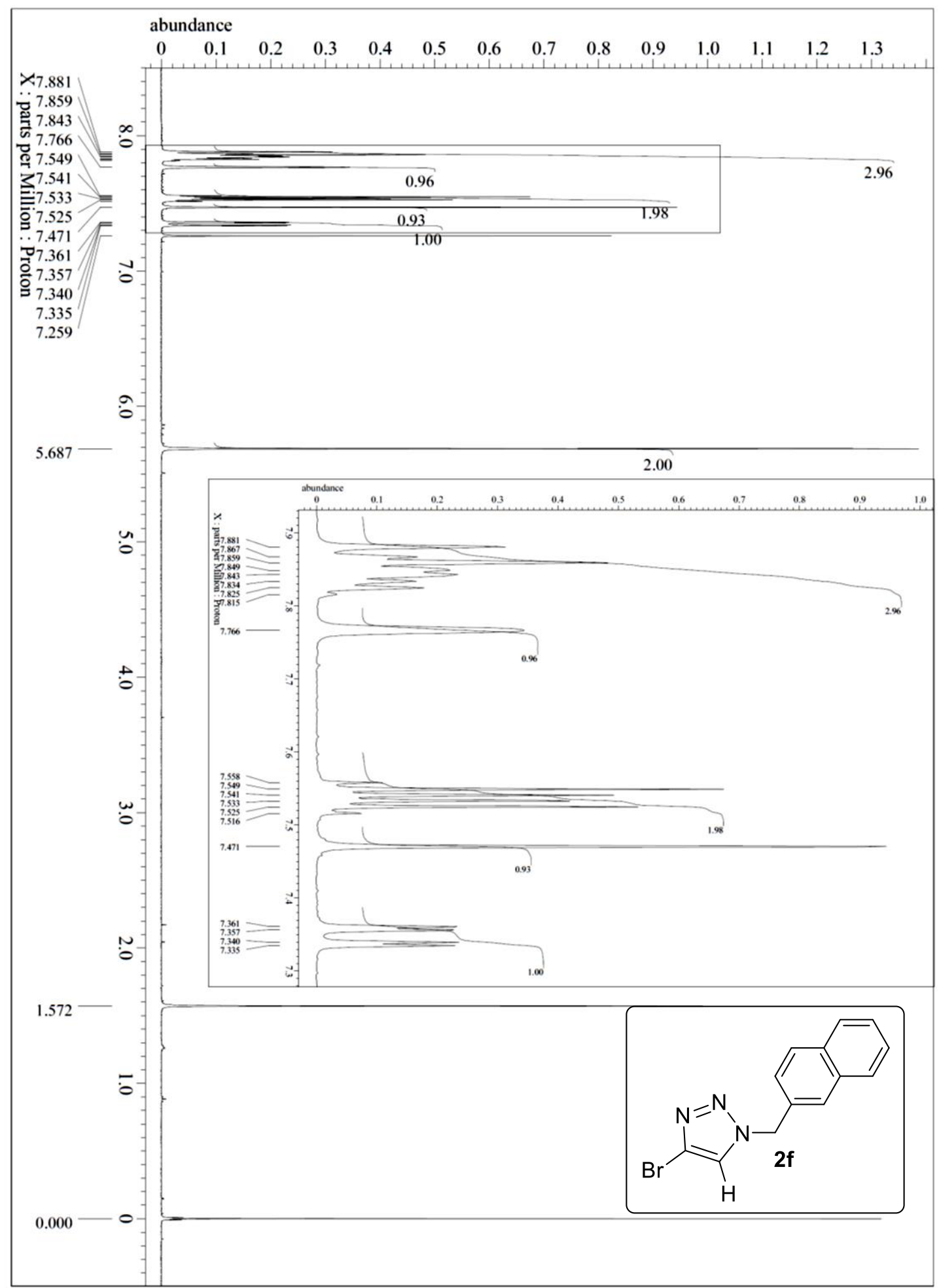




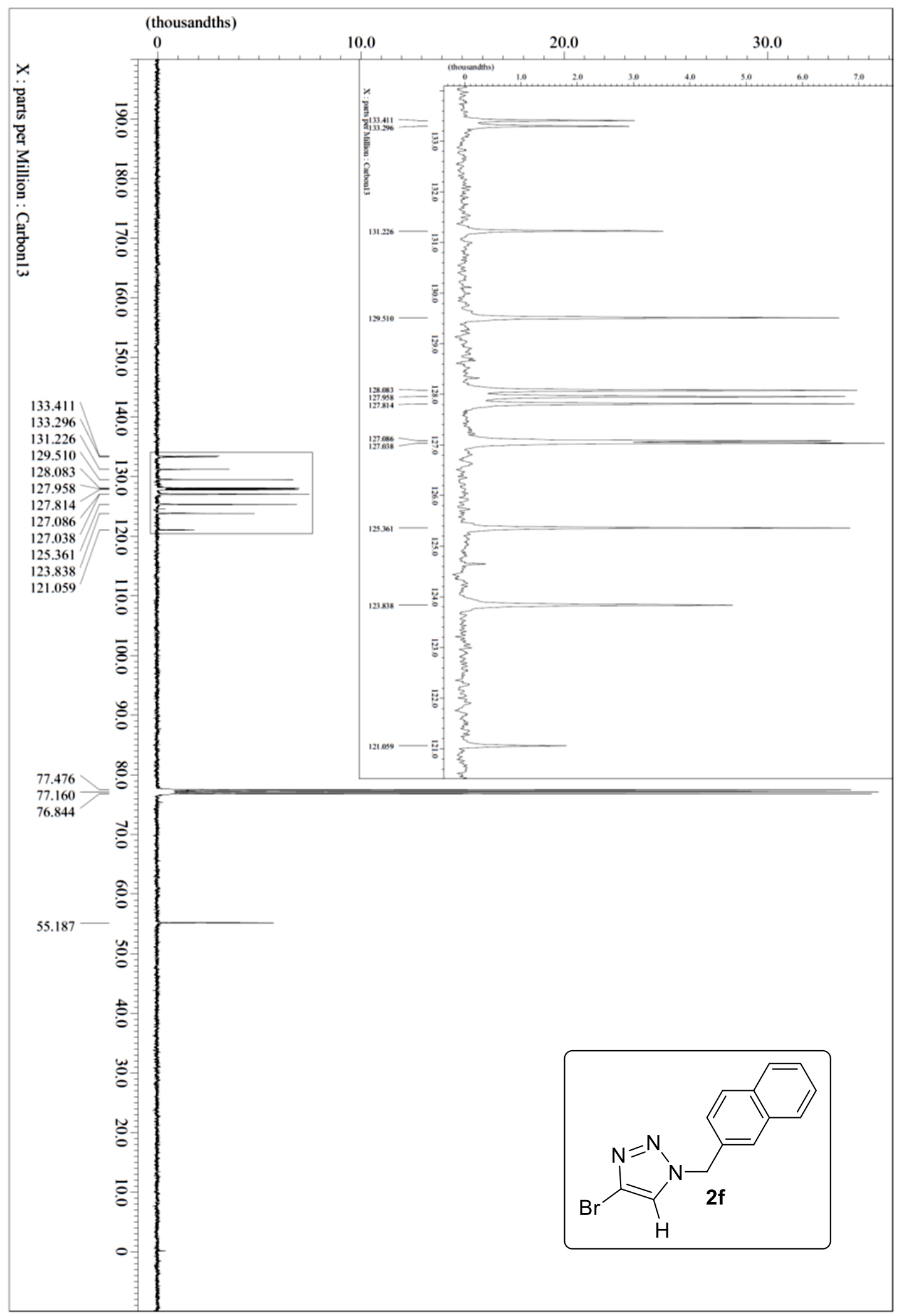

Figure S9. ${ }^{1} \mathrm{H}(400 \mathrm{MHz})$ and ${ }^{13} \mathrm{C}\left\{{ }^{1} \mathrm{H}\right\} \mathrm{NMR}(101 \mathrm{MHz})$ spectra of $\mathbf{2 f}\left(\mathrm{CDCl}_{3}, \mathrm{rt}\right)$ 


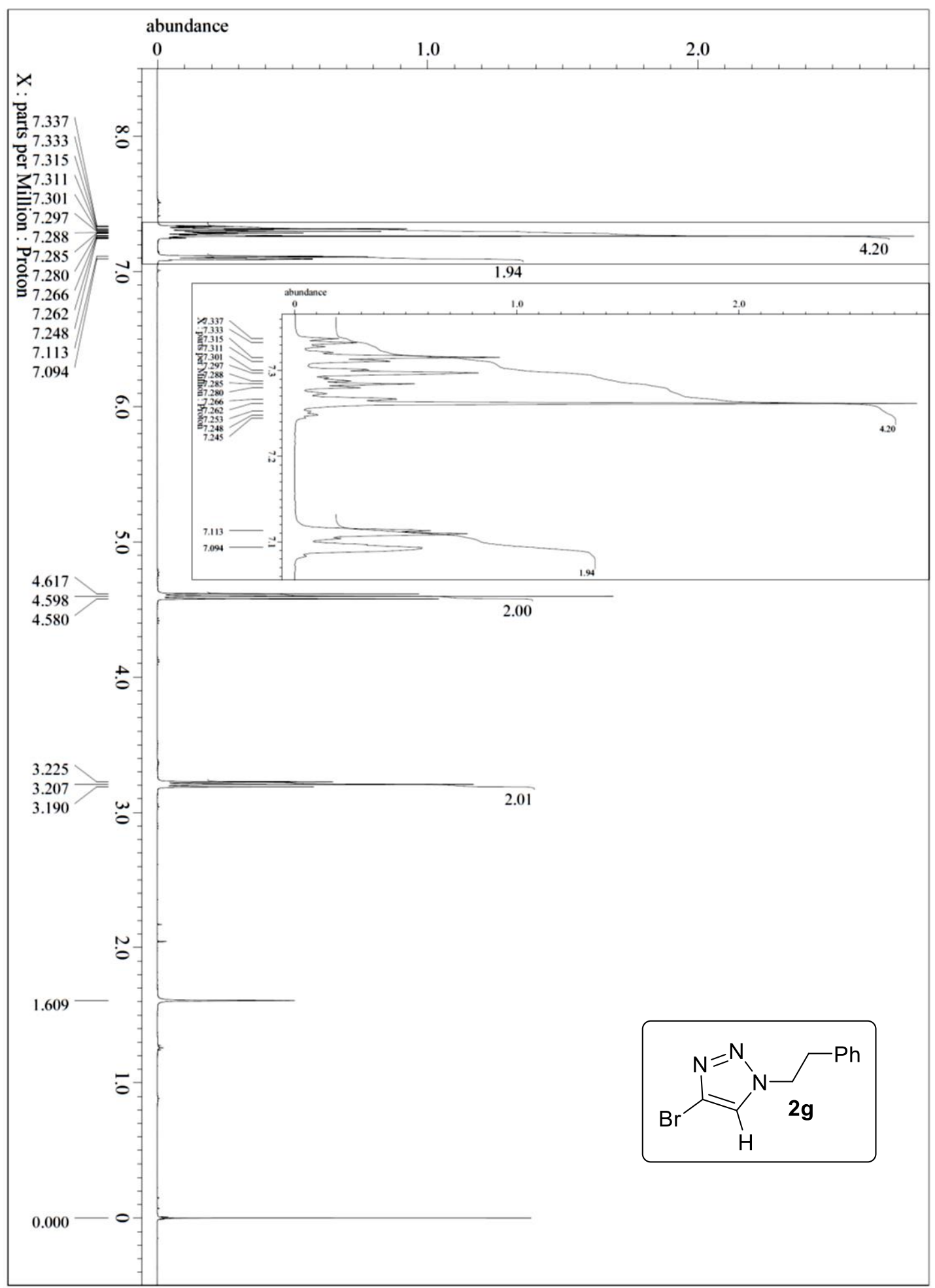




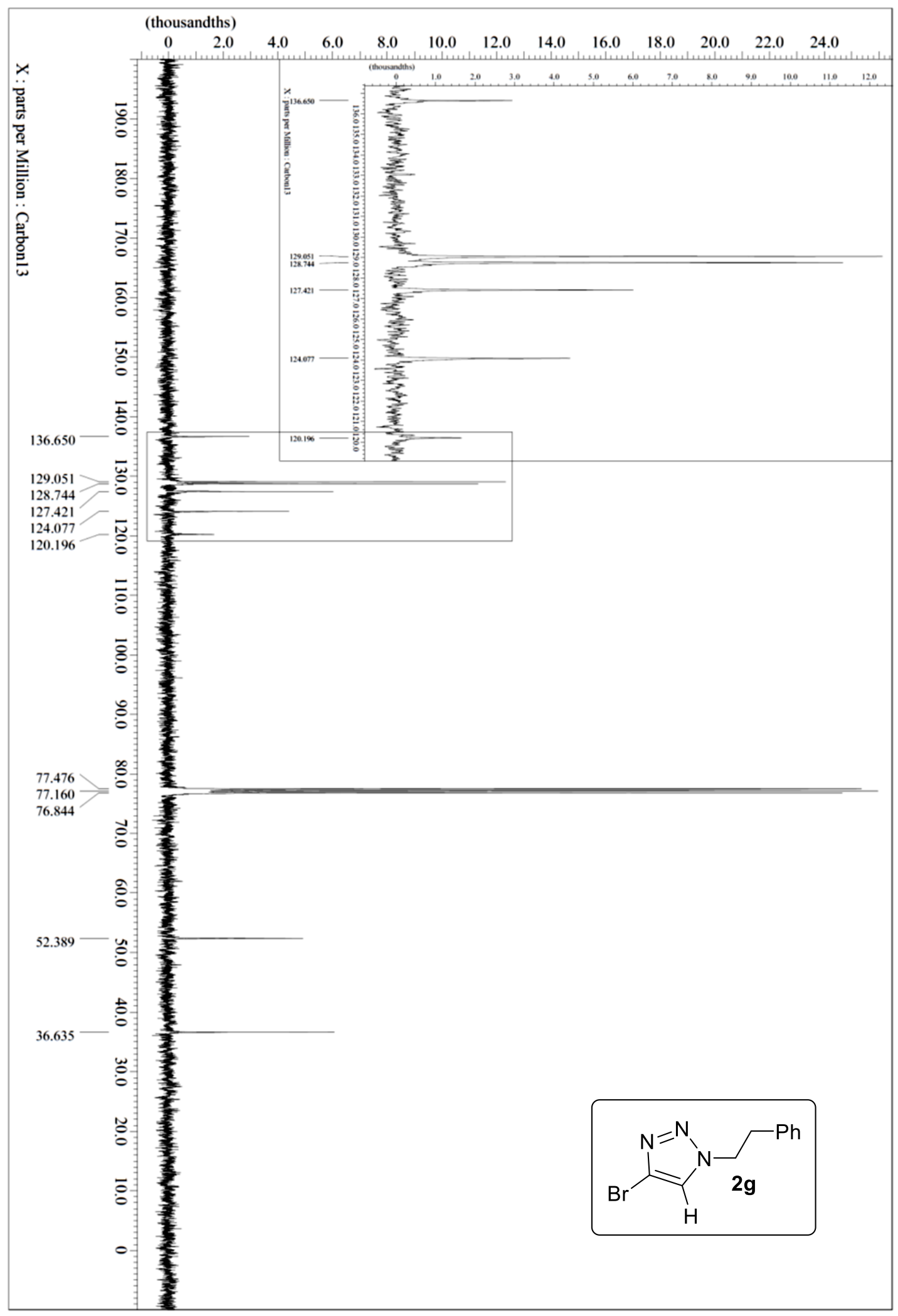

Figure S10. ${ }^{1} \mathrm{H}(400 \mathrm{MHz})$ and ${ }^{13} \mathrm{C}\left\{{ }^{1} \mathrm{H}\right\} \mathrm{NMR}(101 \mathrm{MHz})$ spectra of $\mathbf{2 g}\left(\mathrm{CDCl}_{3}, \mathrm{rt}\right)$ 


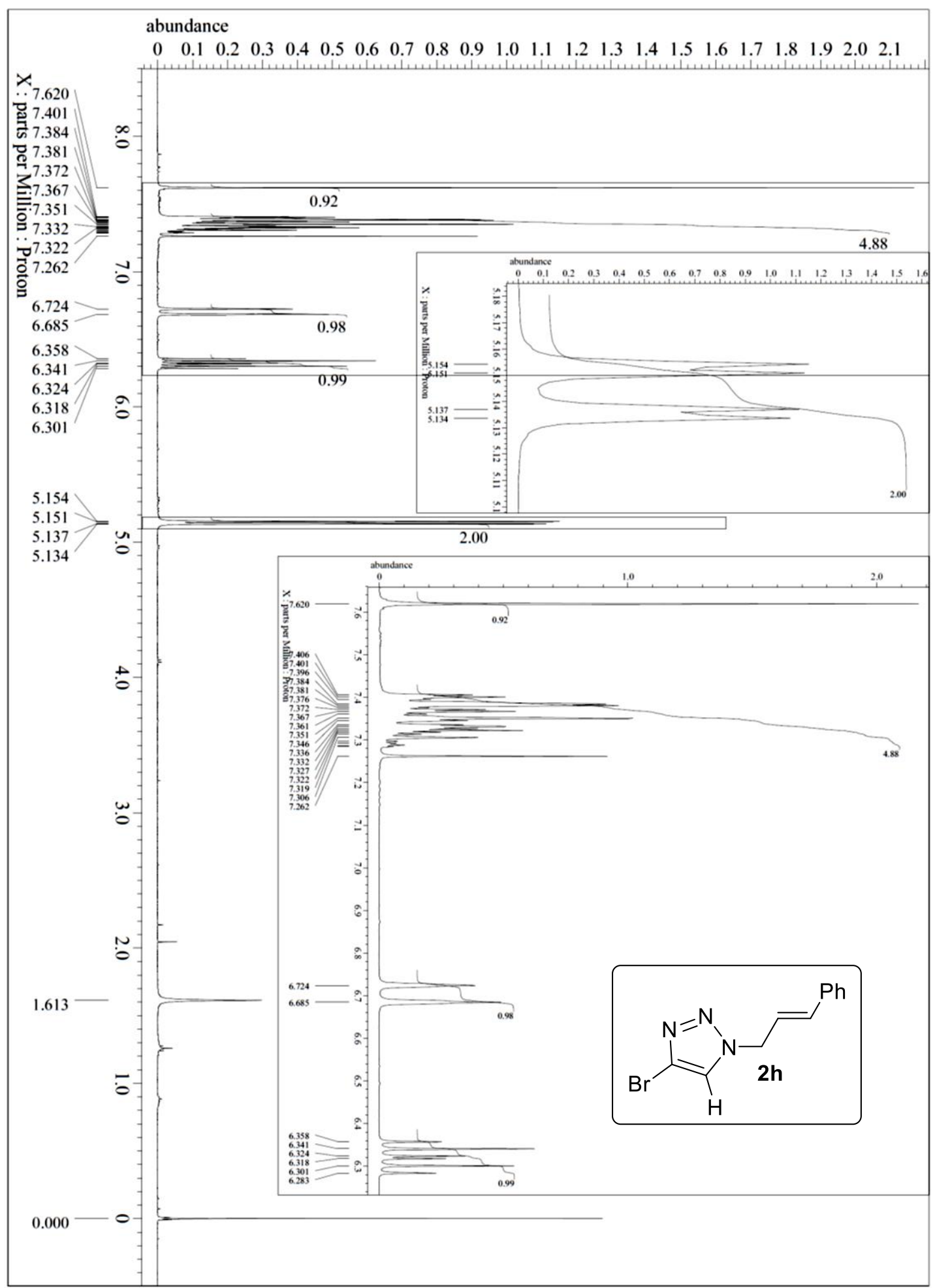




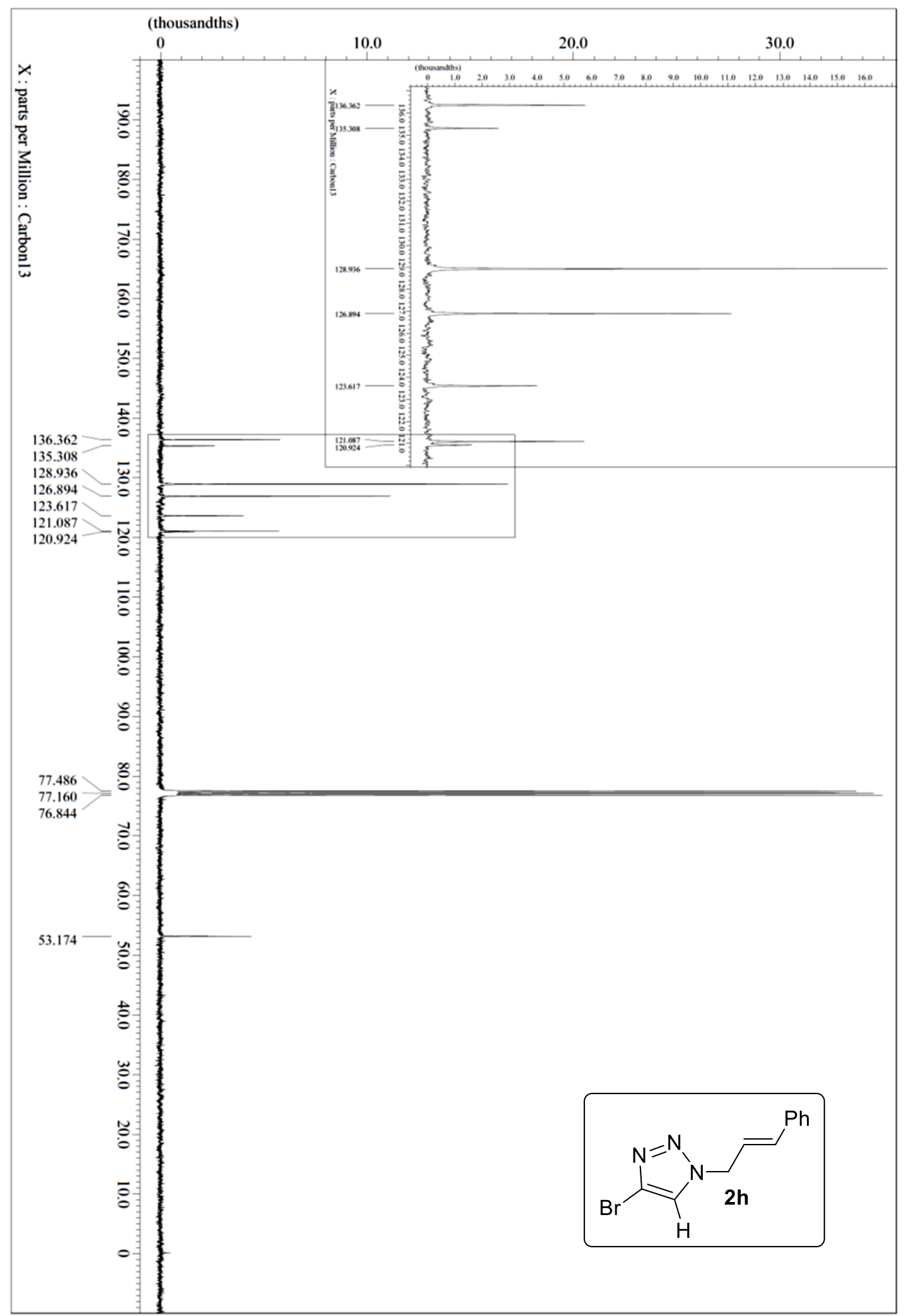

Figure S11. ${ }^{1} \mathrm{H}(400 \mathrm{MHz})$ and ${ }^{13} \mathrm{C}\left\{{ }^{1} \mathrm{H}\right\} \mathrm{NMR}(101 \mathrm{MHz})$ spectra of $\mathbf{2 h}\left(\mathrm{CDCl}_{3}, \mathrm{rt}\right)$ 


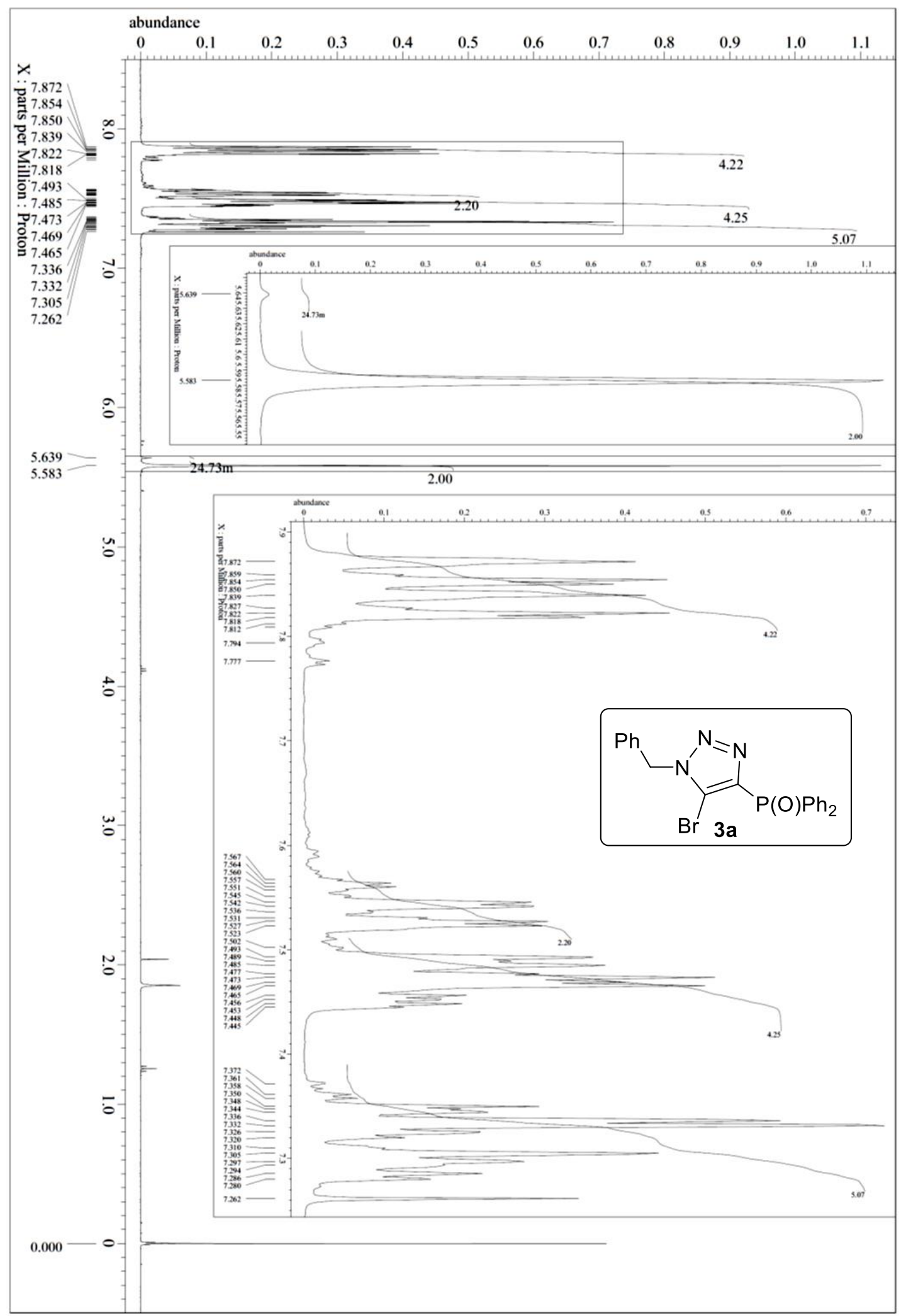




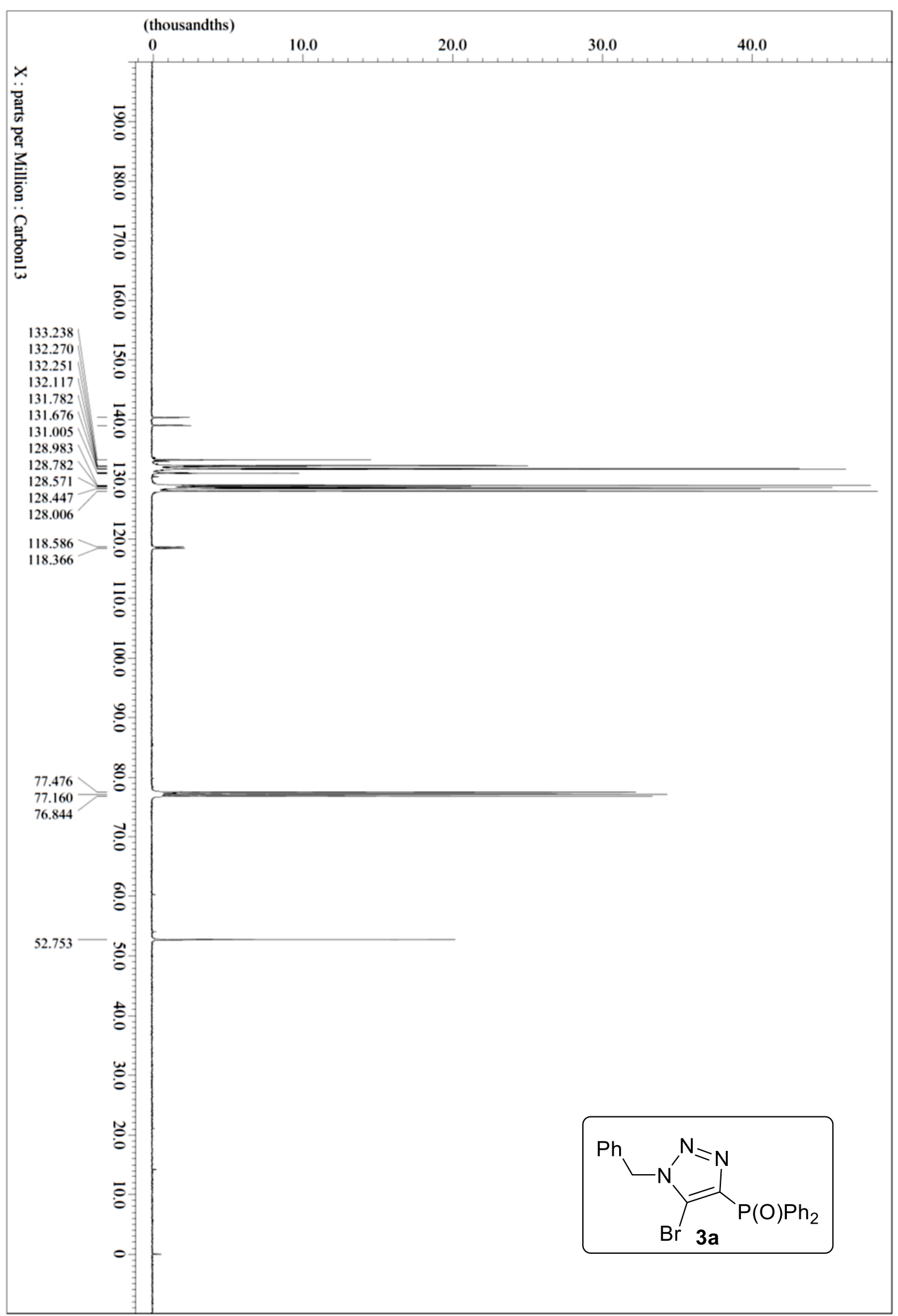




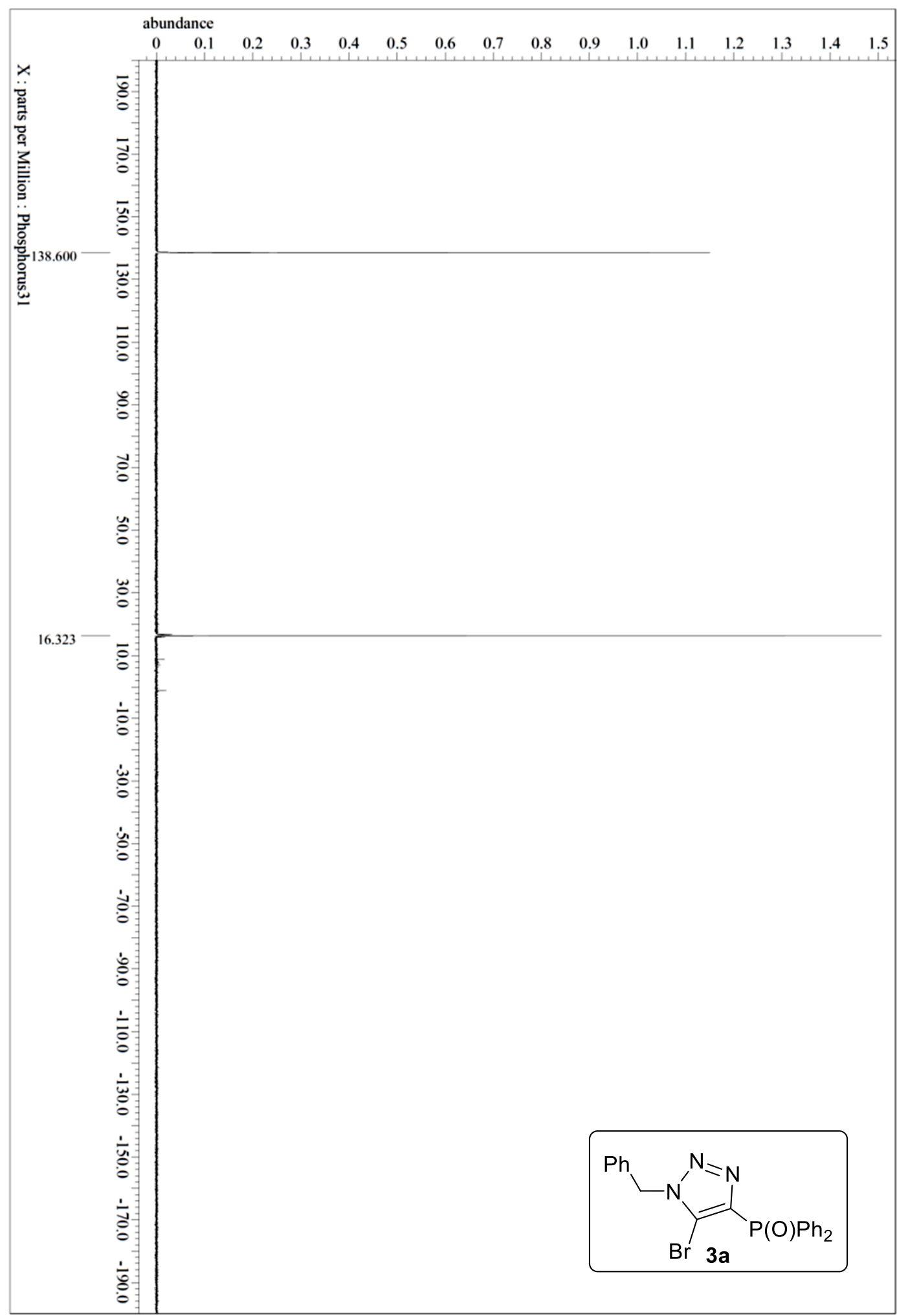

Figure S12. ${ }^{1} \mathrm{H}(400 \mathrm{MHz}),{ }^{13} \mathrm{C}\left\{{ }^{1} \mathrm{H}\right\}$ NMR $(101 \mathrm{MHz})$ and ${ }^{31} \mathrm{P}\left\{{ }^{1} \mathrm{H}\right\}(162 \mathrm{MHz})$ spectra of 3a $\left(\mathrm{CDCl}_{3}, \mathrm{rt}\right)$ 


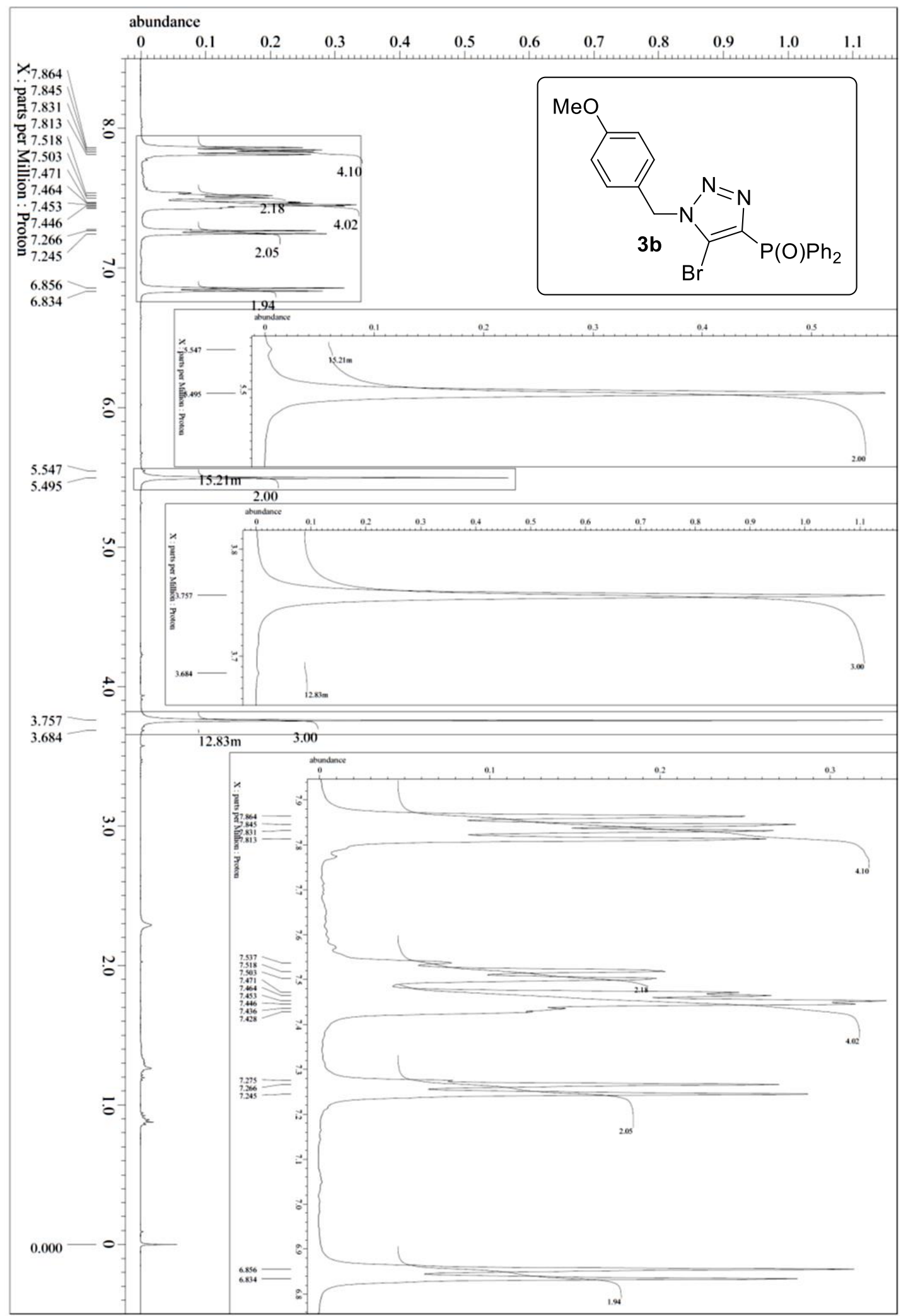




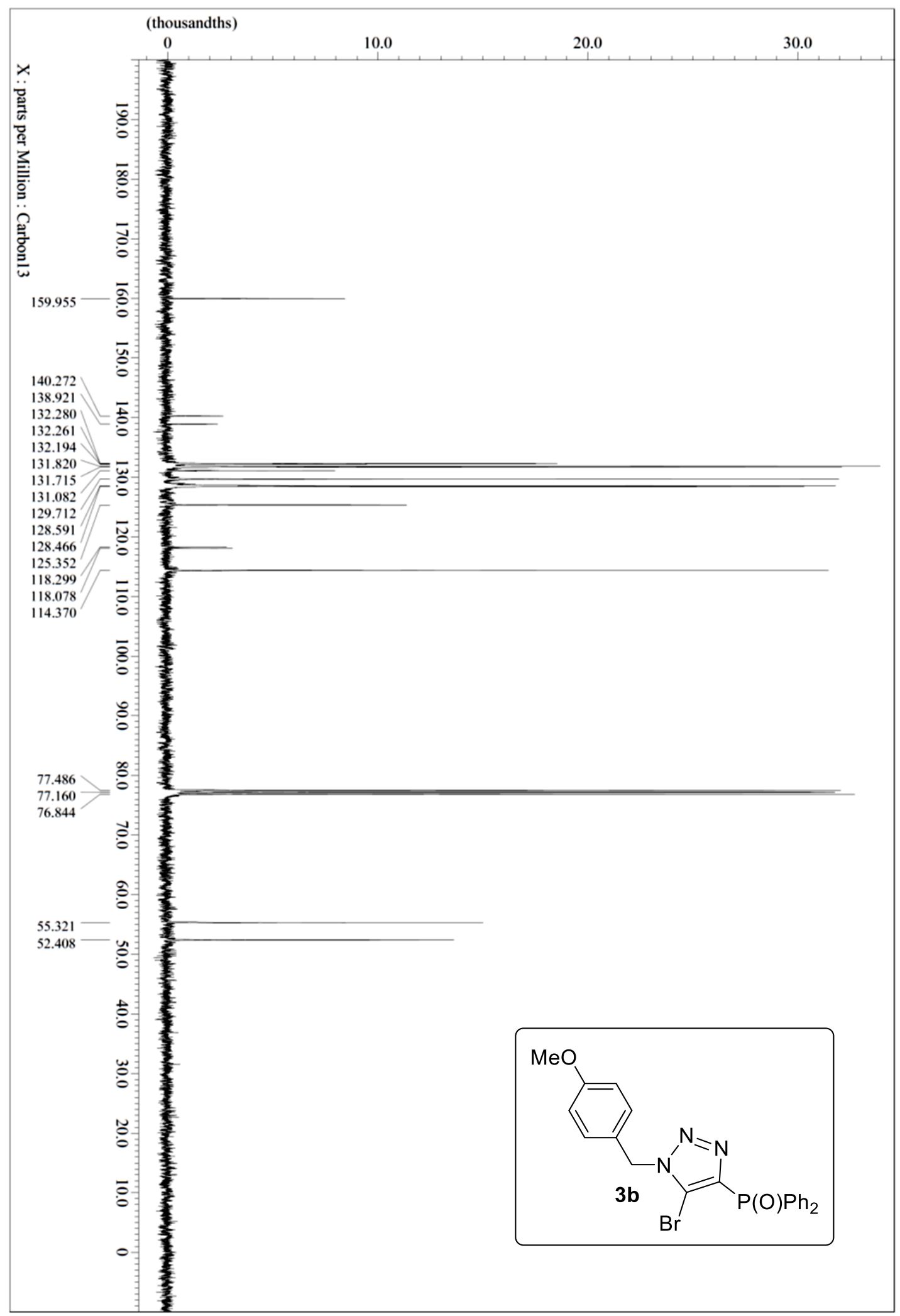




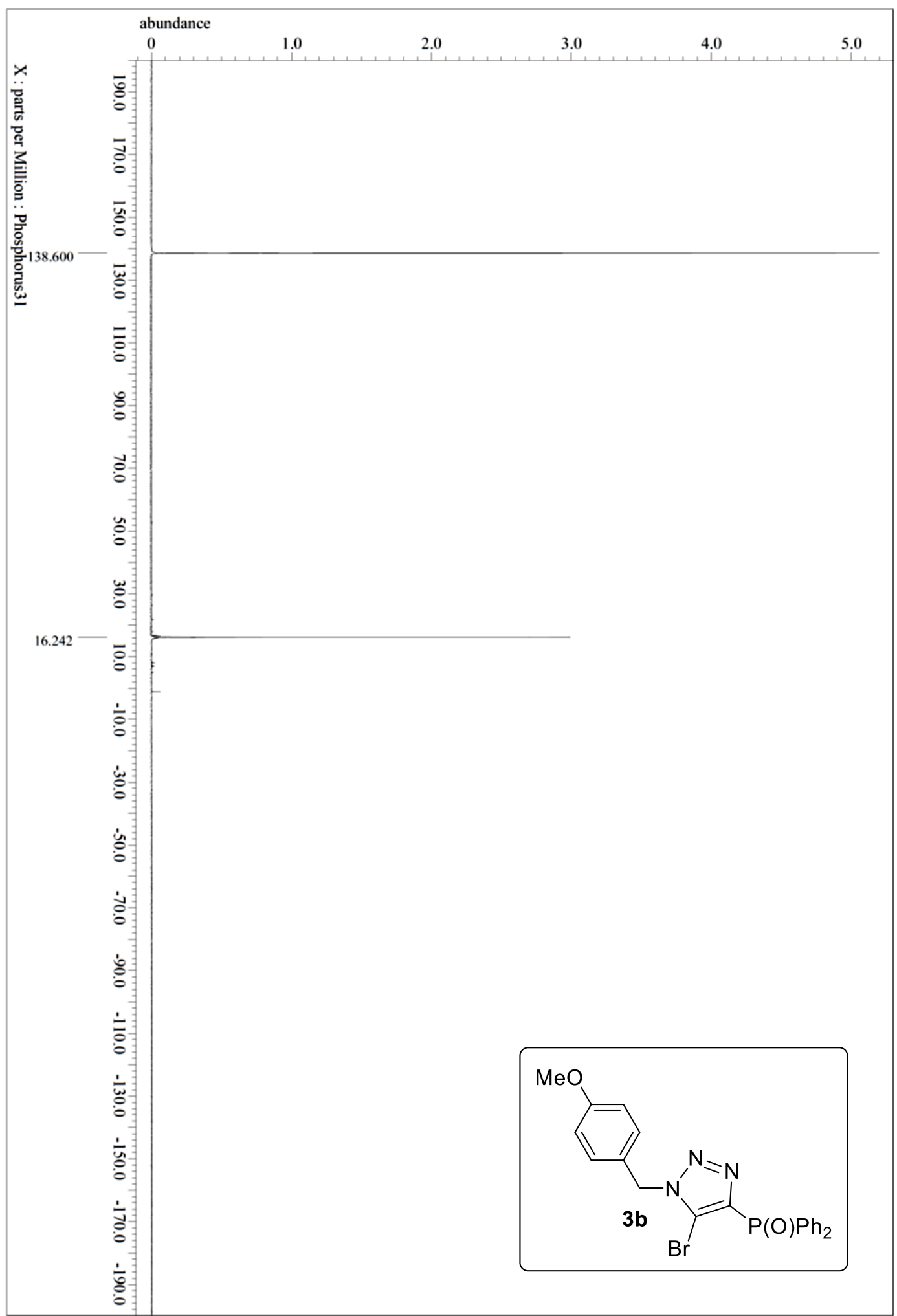

Figure S13. ${ }^{1} \mathrm{H}(400 \mathrm{MHz}),{ }^{13} \mathrm{C}\left\{{ }^{1} \mathrm{H}\right\}$ NMR $(101 \mathrm{MHz})$ and ${ }^{31} \mathrm{P}\left\{{ }^{1} \mathrm{H}\right\}(162 \mathrm{MHz})$ spectra of $\mathbf{3 b}$ $\left(\mathrm{CDCl}_{3}, \mathrm{rt}\right)$ 


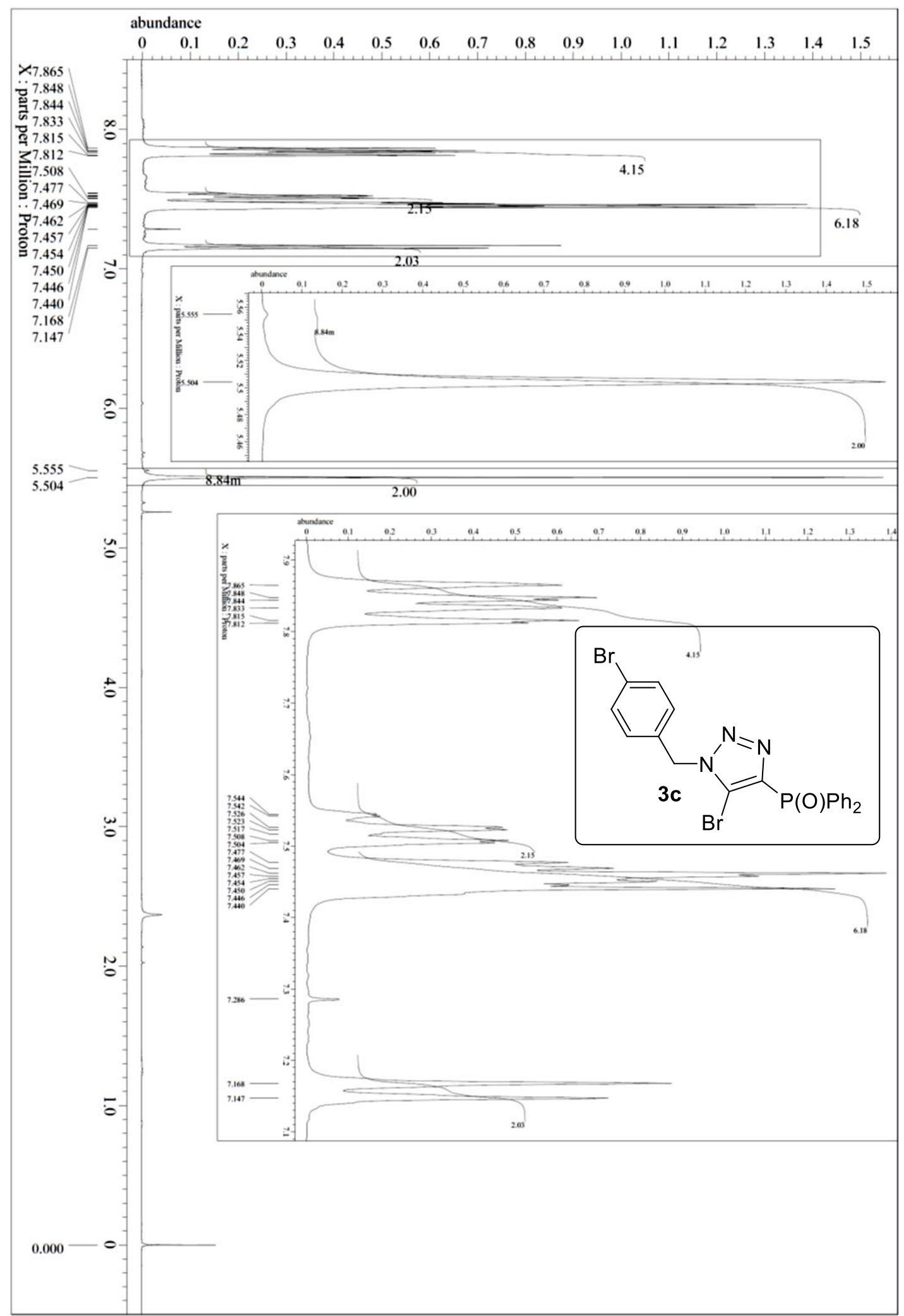




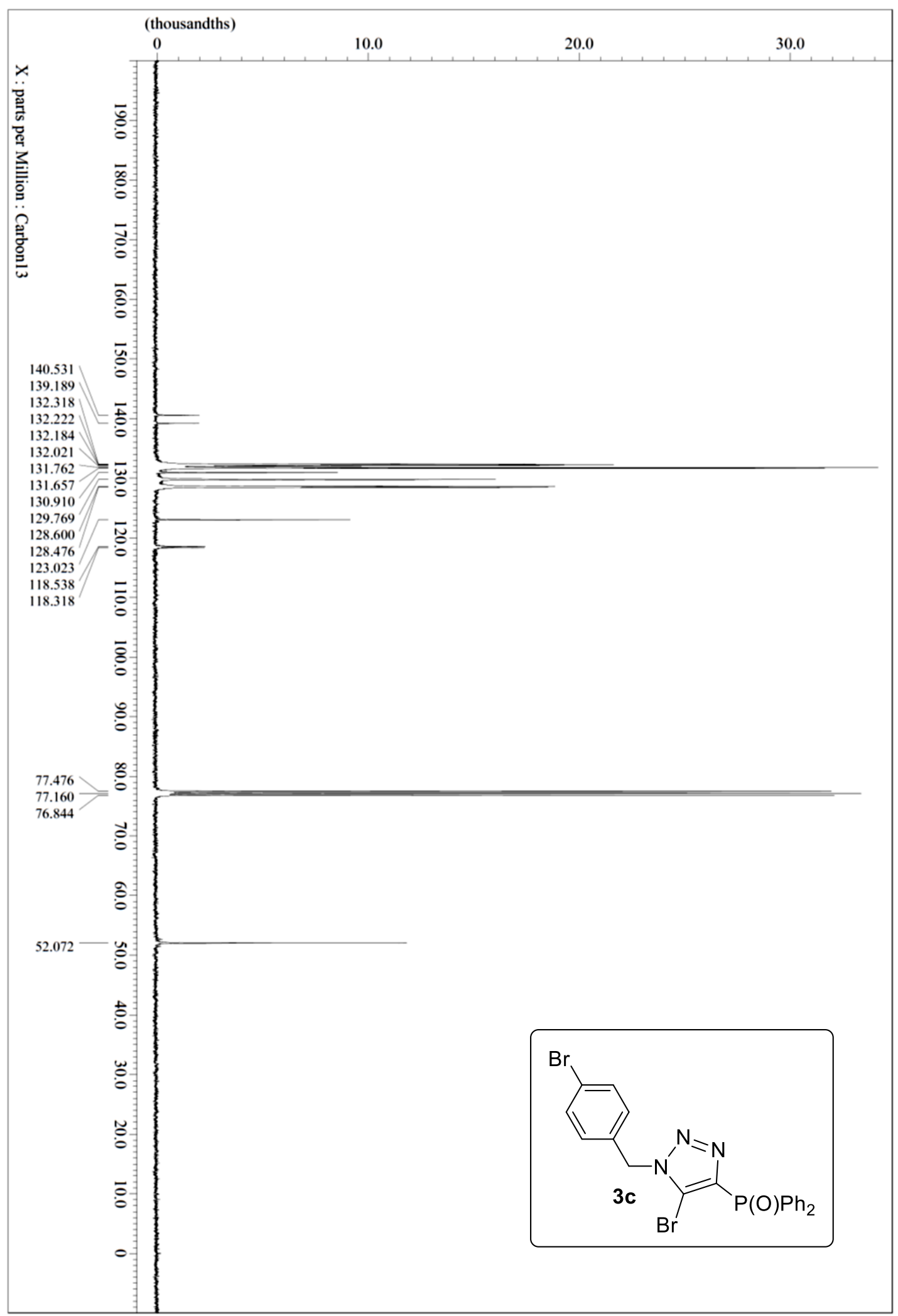




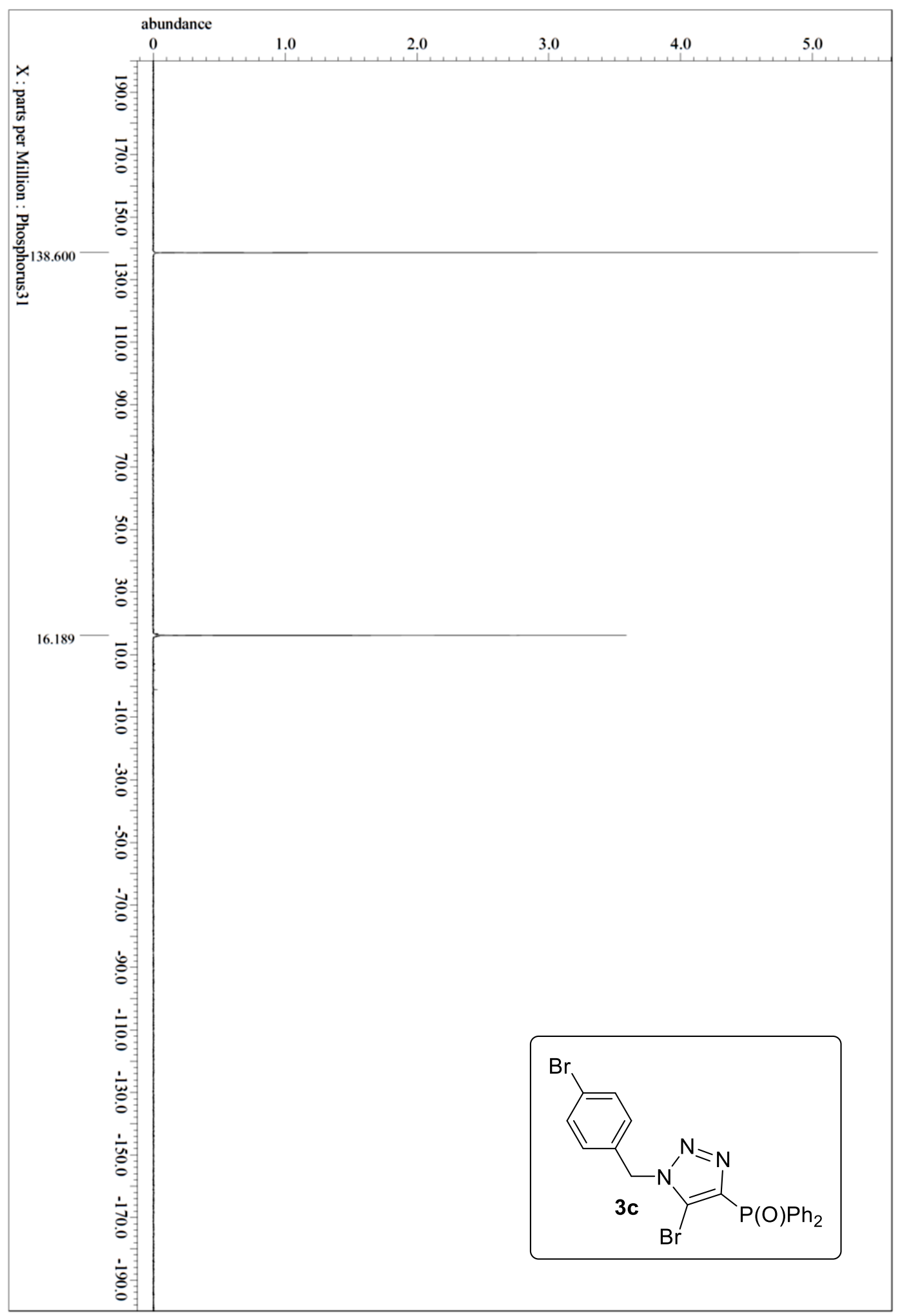

Figure S14. ${ }^{1} \mathrm{H}(400 \mathrm{MHz}),{ }^{13} \mathrm{C}\left\{{ }^{1} \mathrm{H}\right\}$ NMR $(101 \mathrm{MHz})$ and ${ }^{31} \mathrm{P}\left\{{ }^{1} \mathrm{H}\right\}(162 \mathrm{MHz})$ spectra of 3c $\left(\mathrm{CDCl}_{3}, \mathrm{rt}\right)$ 


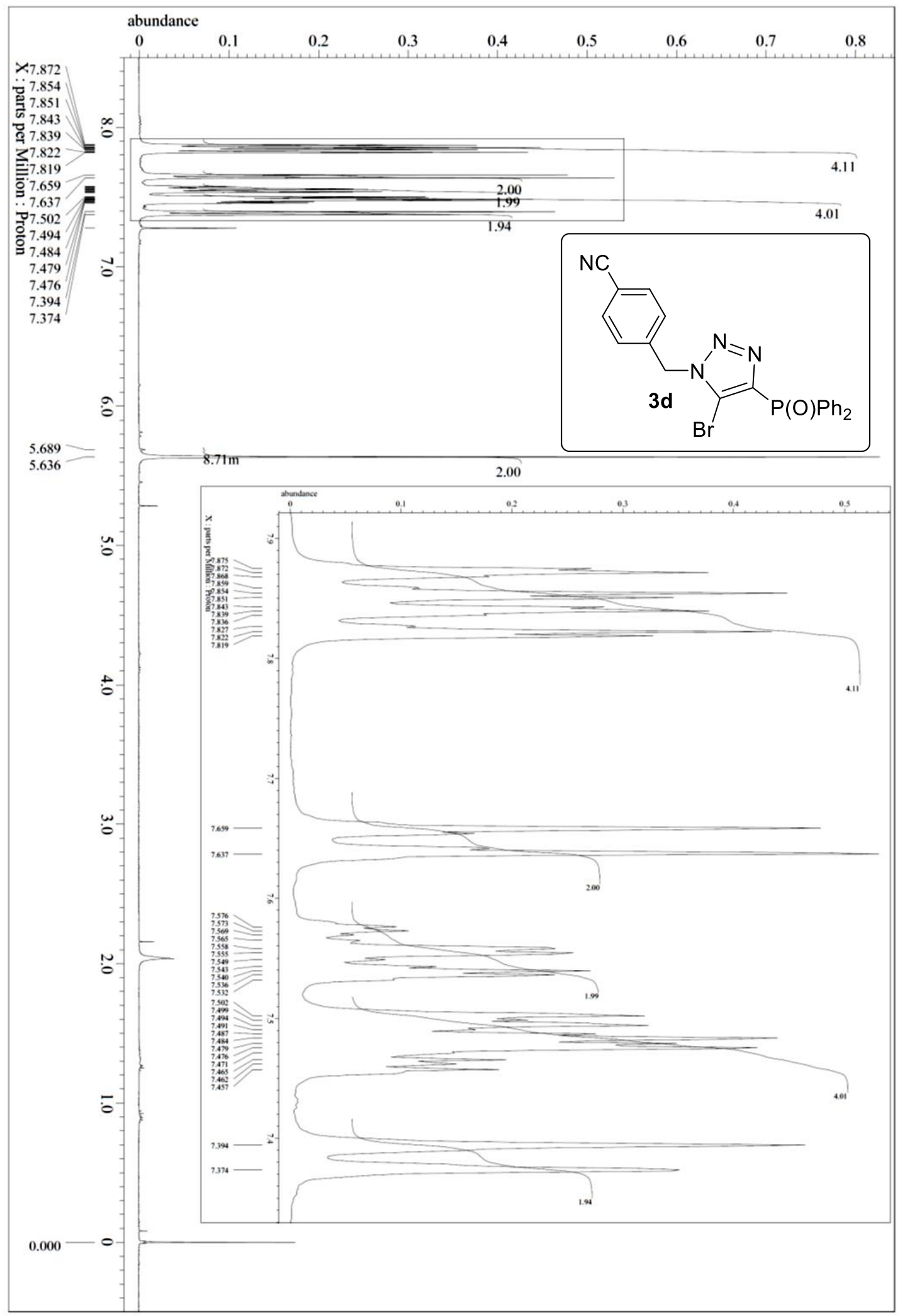




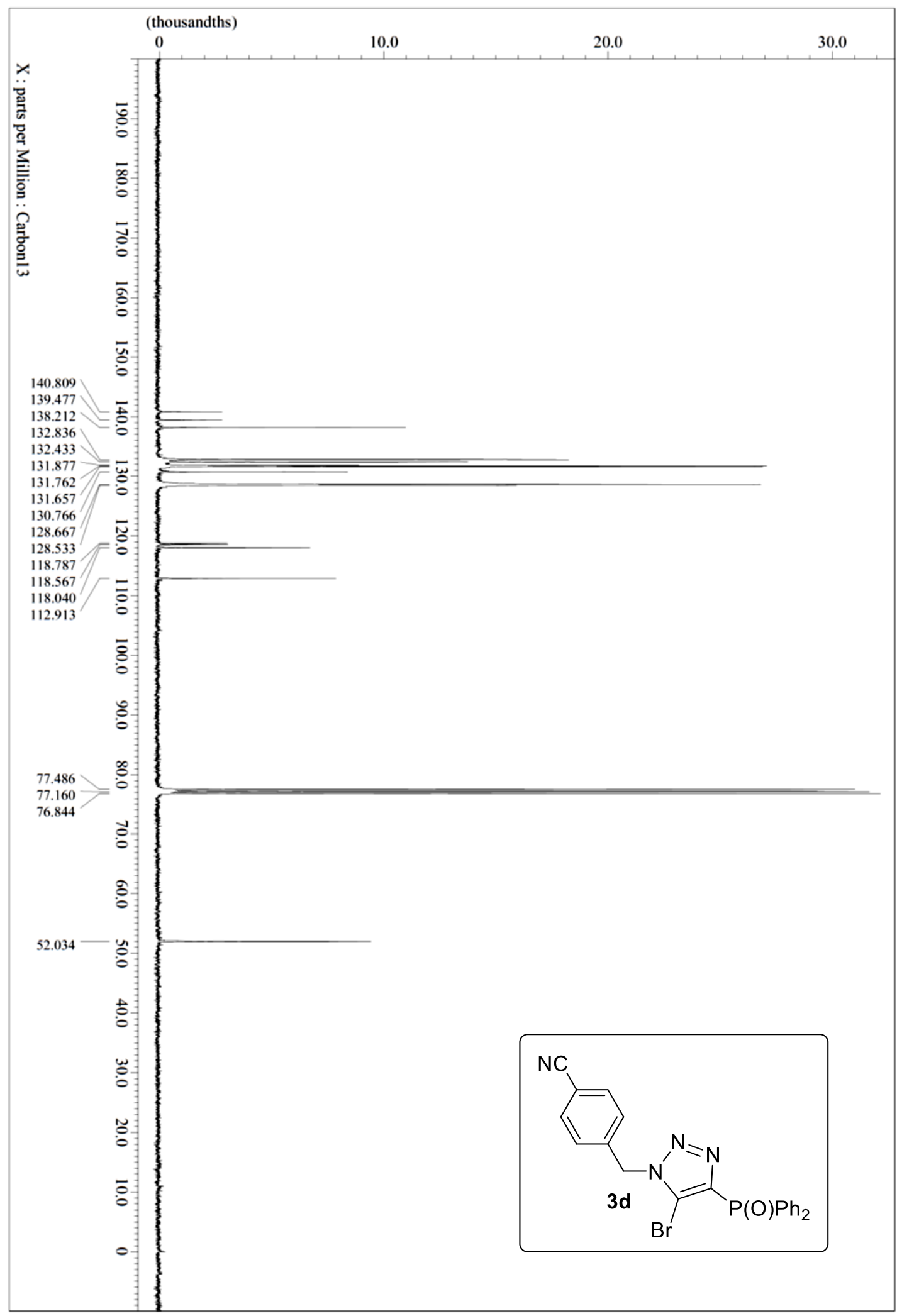




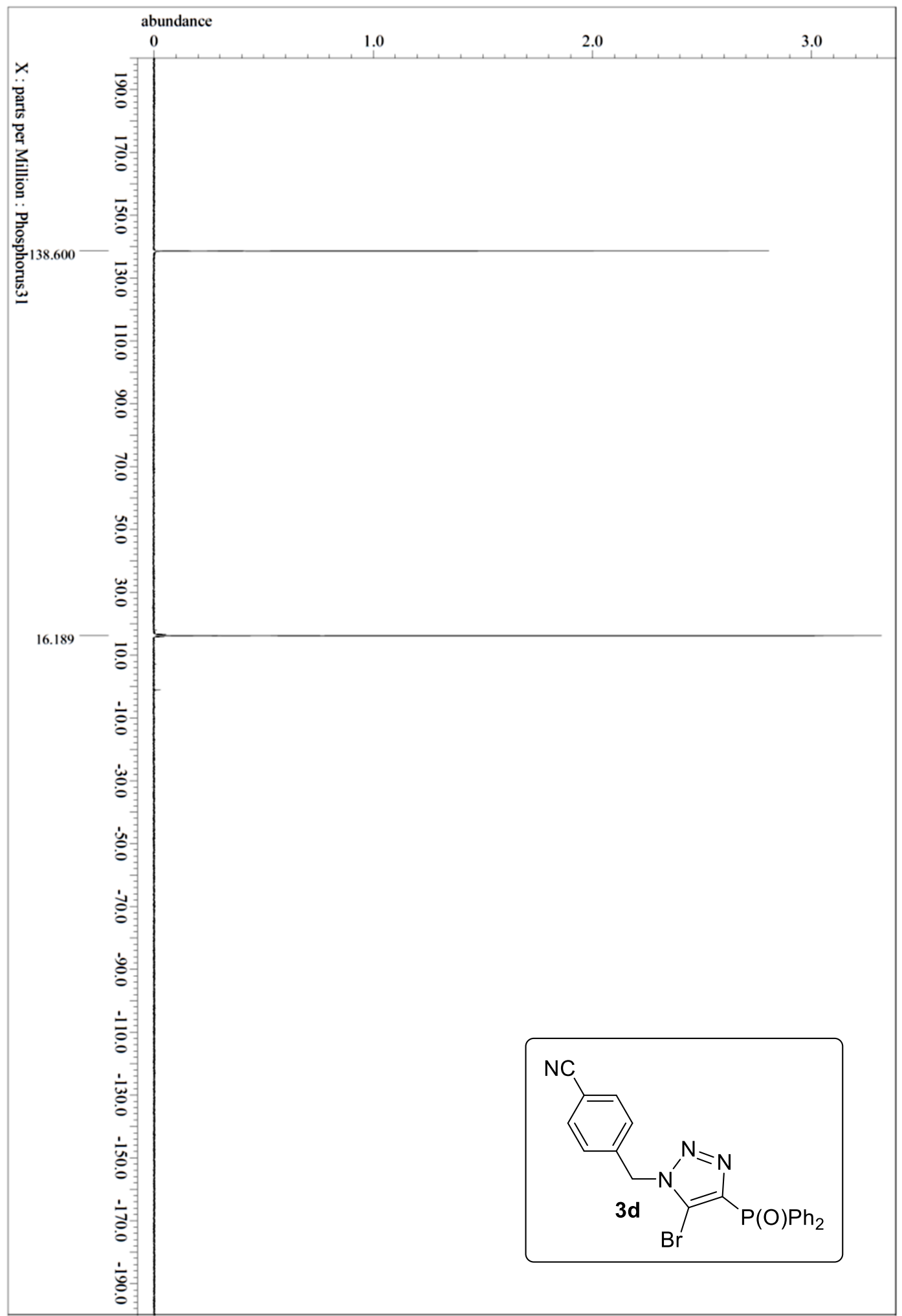

Figure S15. ${ }^{1} \mathrm{H}(400 \mathrm{MHz}),{ }^{13} \mathrm{C}\left\{{ }^{1} \mathrm{H}\right\}$ NMR $(101 \mathrm{MHz})$ and ${ }^{31} \mathrm{P}\left\{{ }^{1} \mathrm{H}\right\}(162 \mathrm{MHz})$ spectra of 3d $\left(\mathrm{CDCl}_{3}, \mathrm{rt}\right)$ 


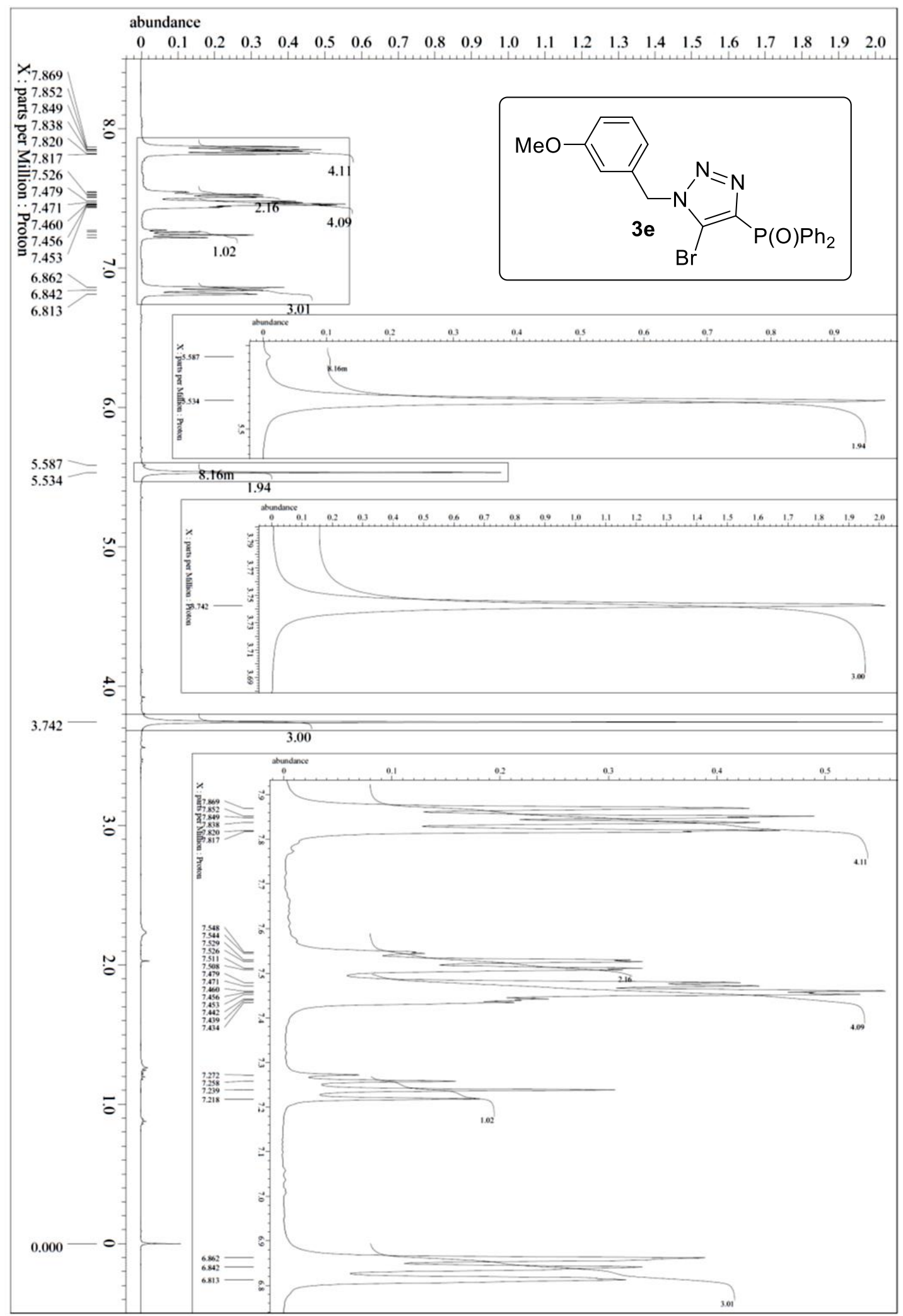




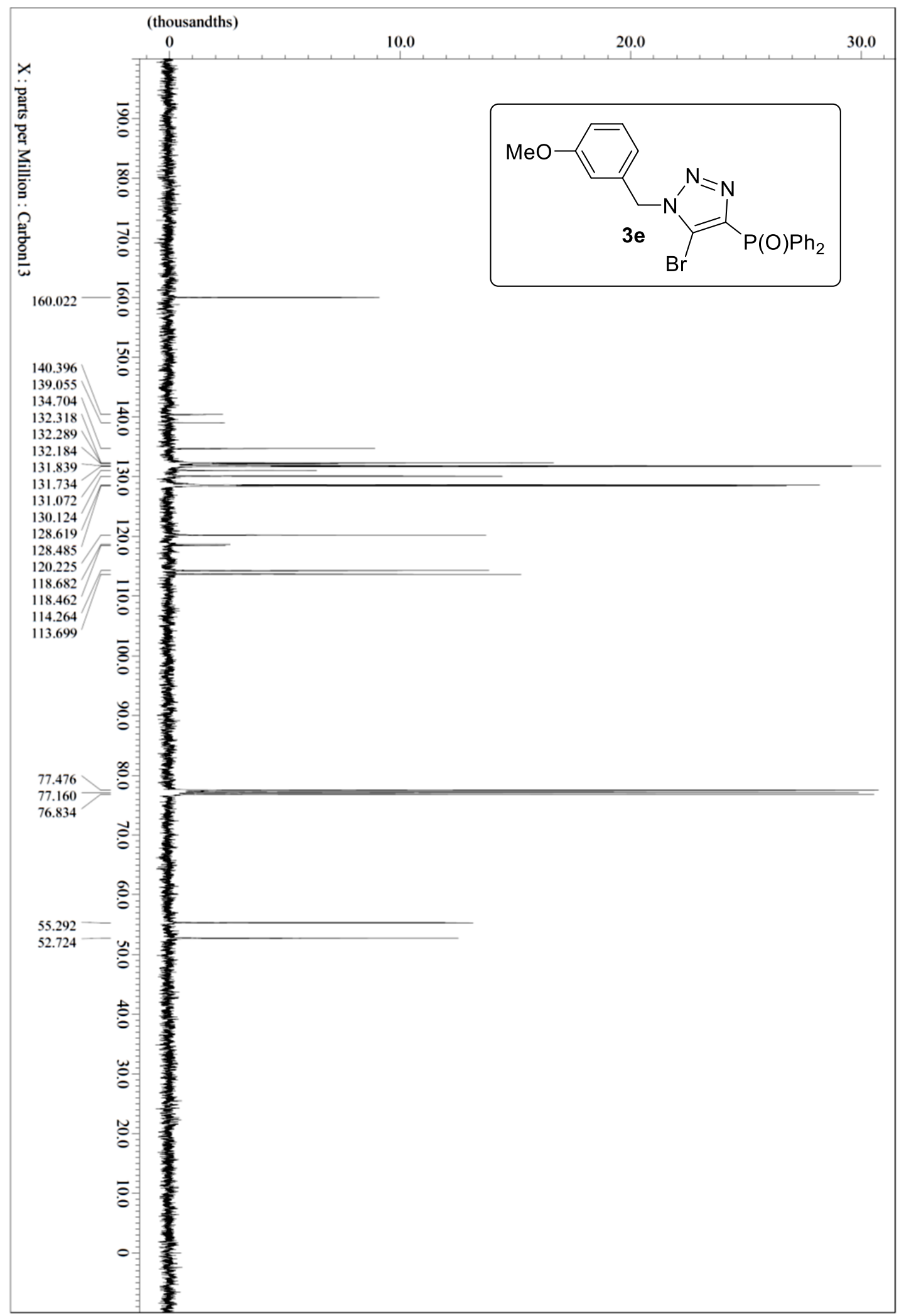




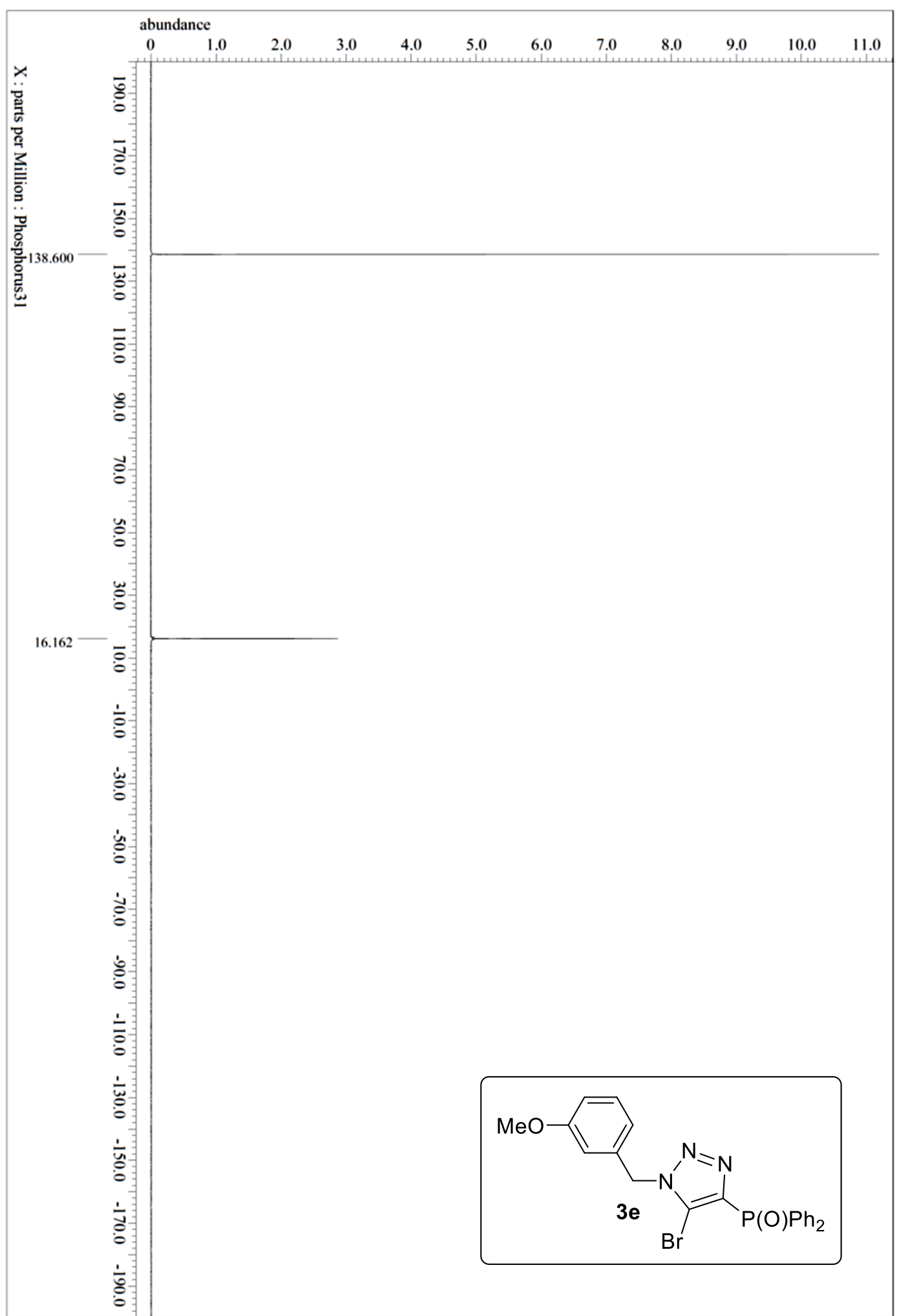

Figure S16. ${ }^{1} \mathrm{H}(400 \mathrm{MHz}),{ }^{13} \mathrm{C}\left\{{ }^{1} \mathrm{H}\right\}$ NMR $(101 \mathrm{MHz})$ and ${ }^{31} \mathrm{P}\left\{{ }^{1} \mathrm{H}\right\}(162 \mathrm{MHz})$ spectra of 3e $\left(\mathrm{CDCl}_{3}, \mathrm{rt}\right)$ 


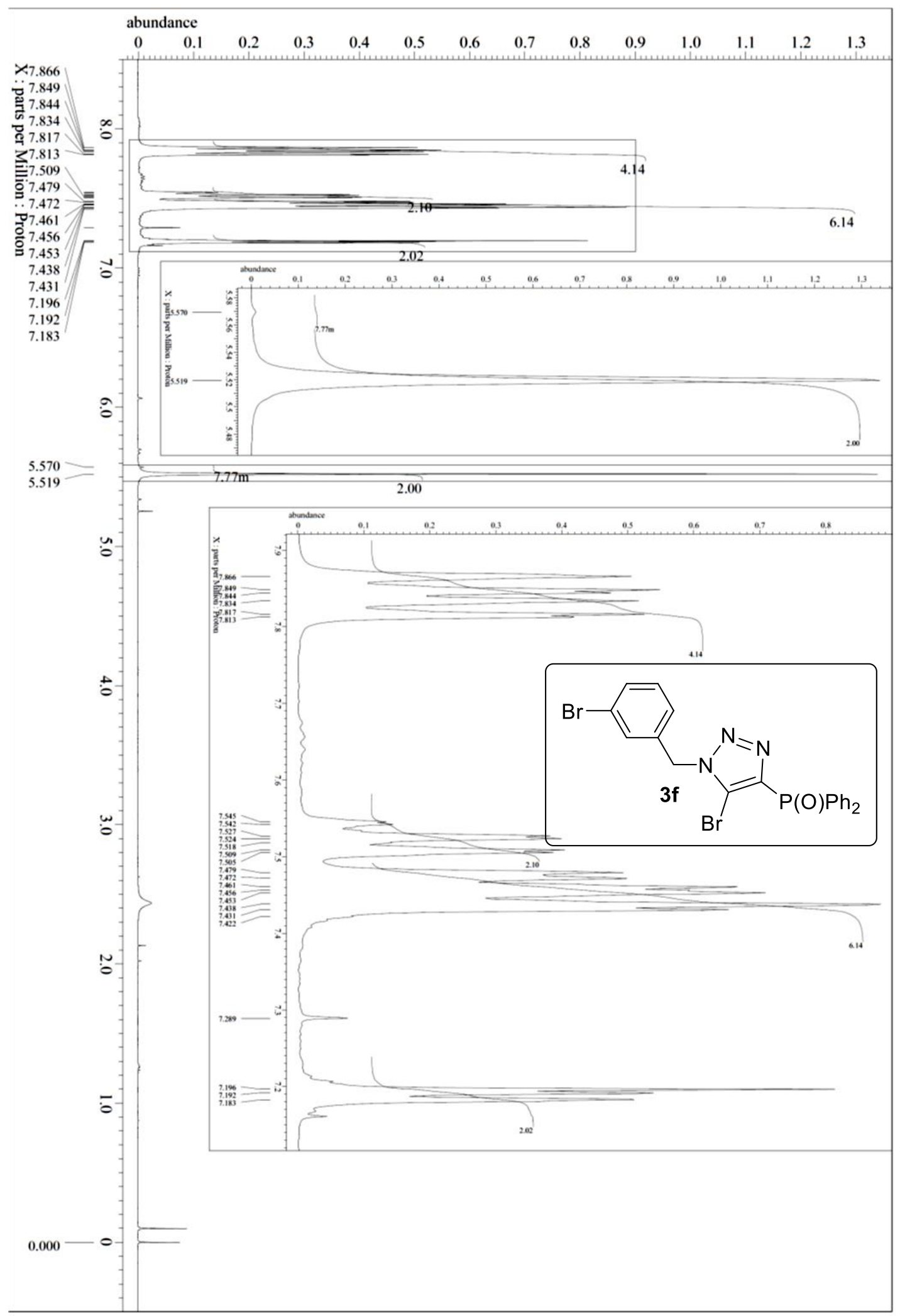




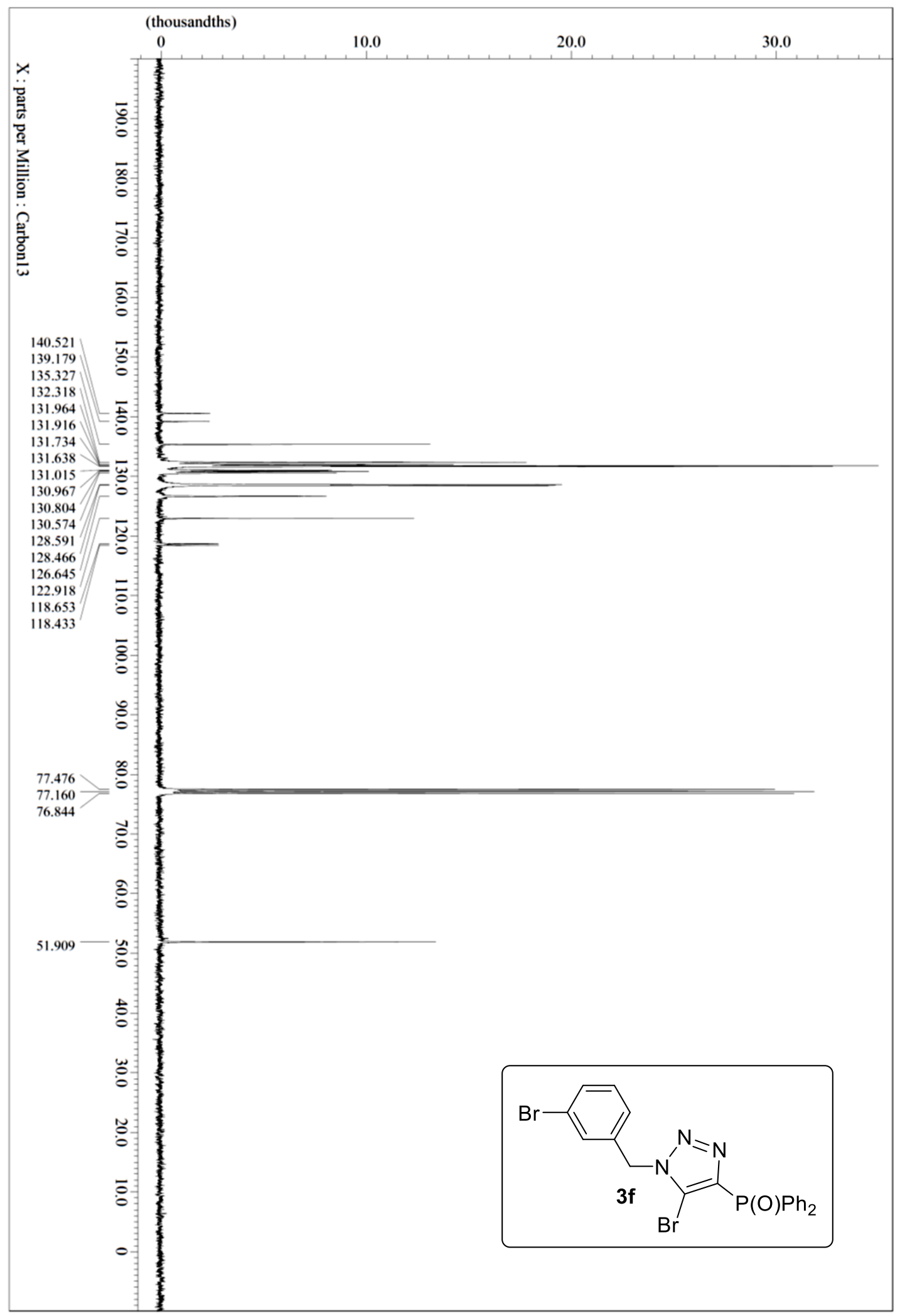




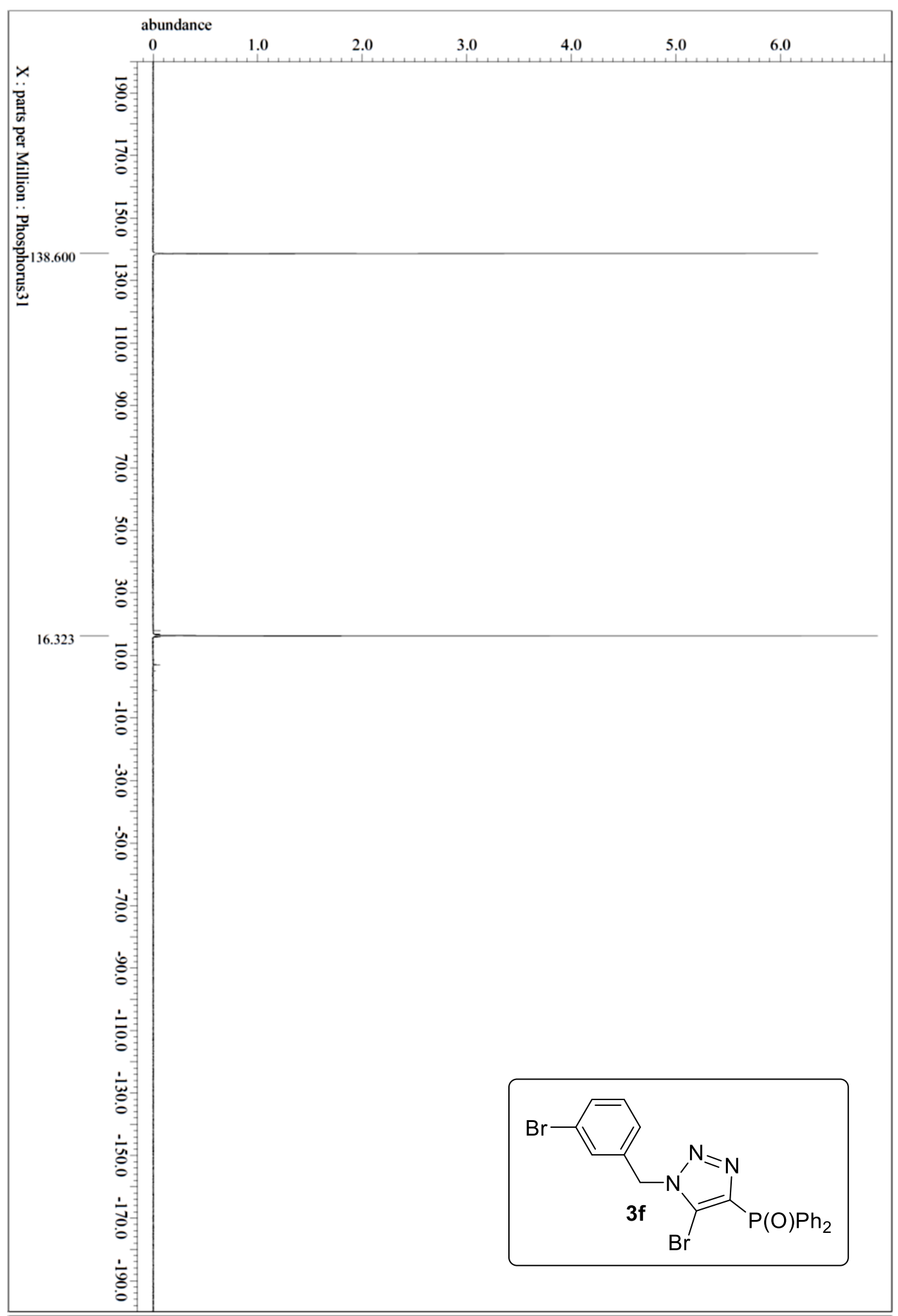

Figure S17. ${ }^{1} \mathrm{H}(400 \mathrm{MHz}),{ }^{13} \mathrm{C}\left\{{ }^{1} \mathrm{H}\right\}$ NMR $(101 \mathrm{MHz})$ and ${ }^{31} \mathrm{P}\left\{{ }^{1} \mathrm{H}\right\}(162 \mathrm{MHz})$ spectra of $\mathbf{3 f}$ $\left(\mathrm{CDCl}_{3}, \mathrm{rt}\right)$ 


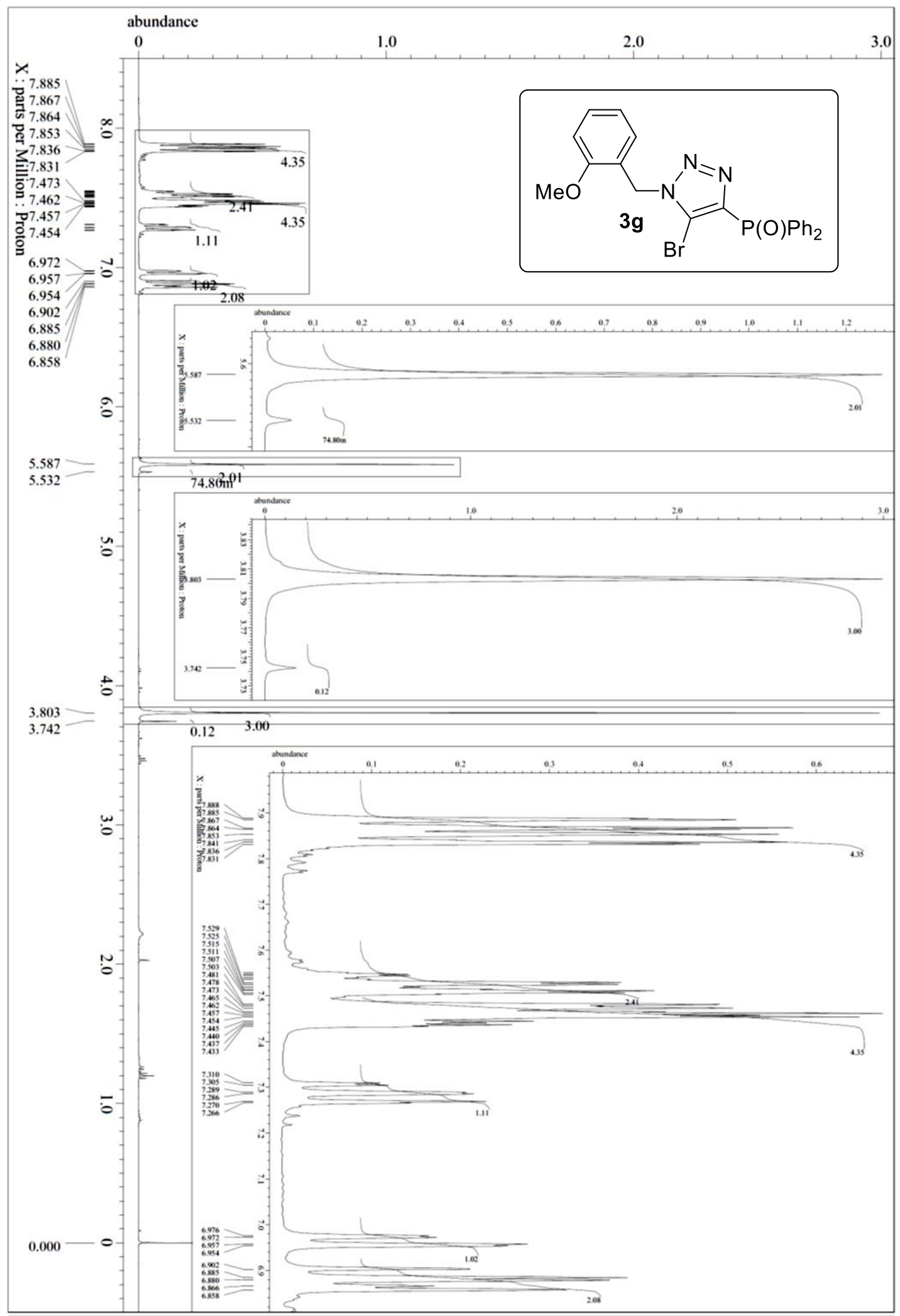




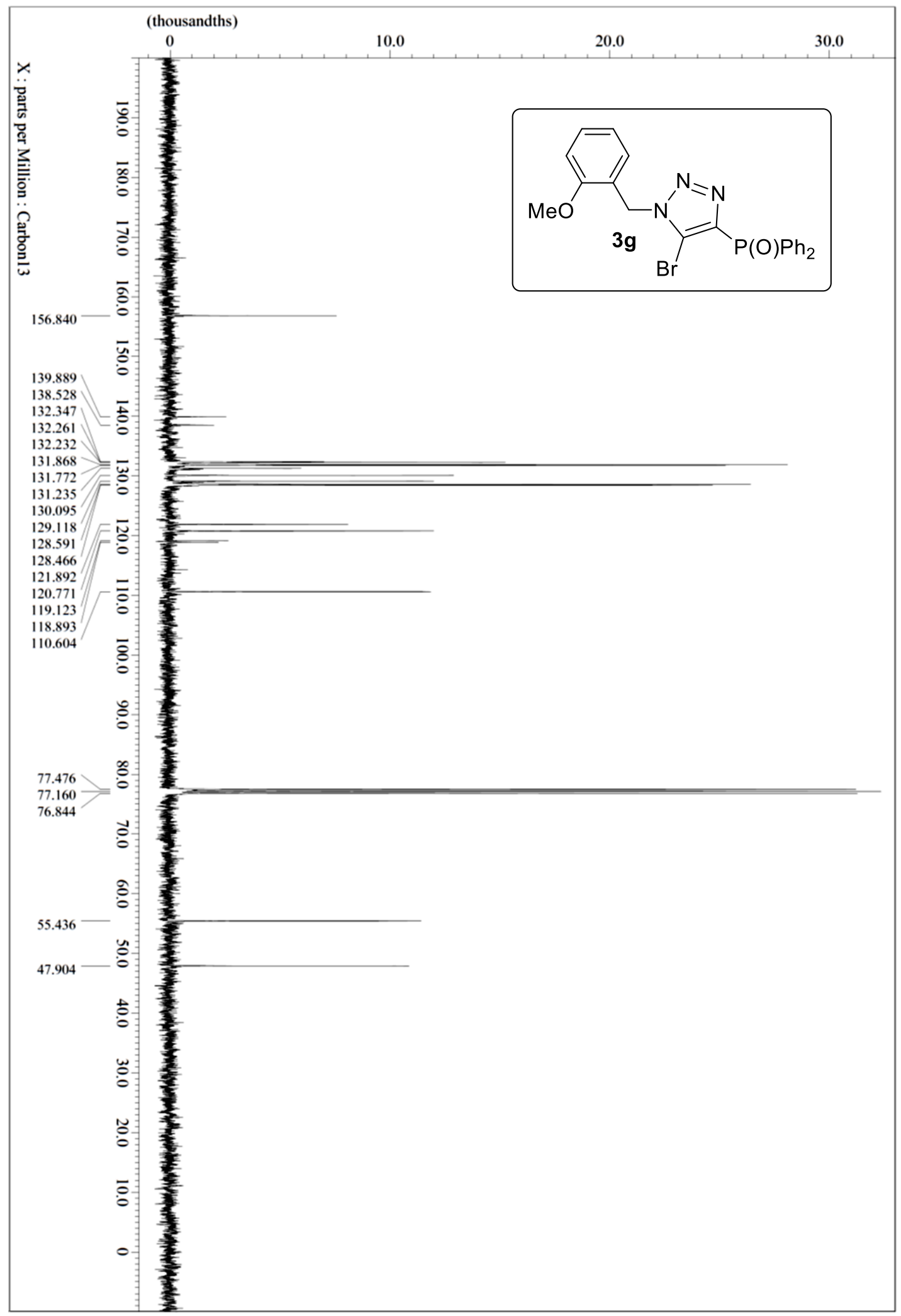




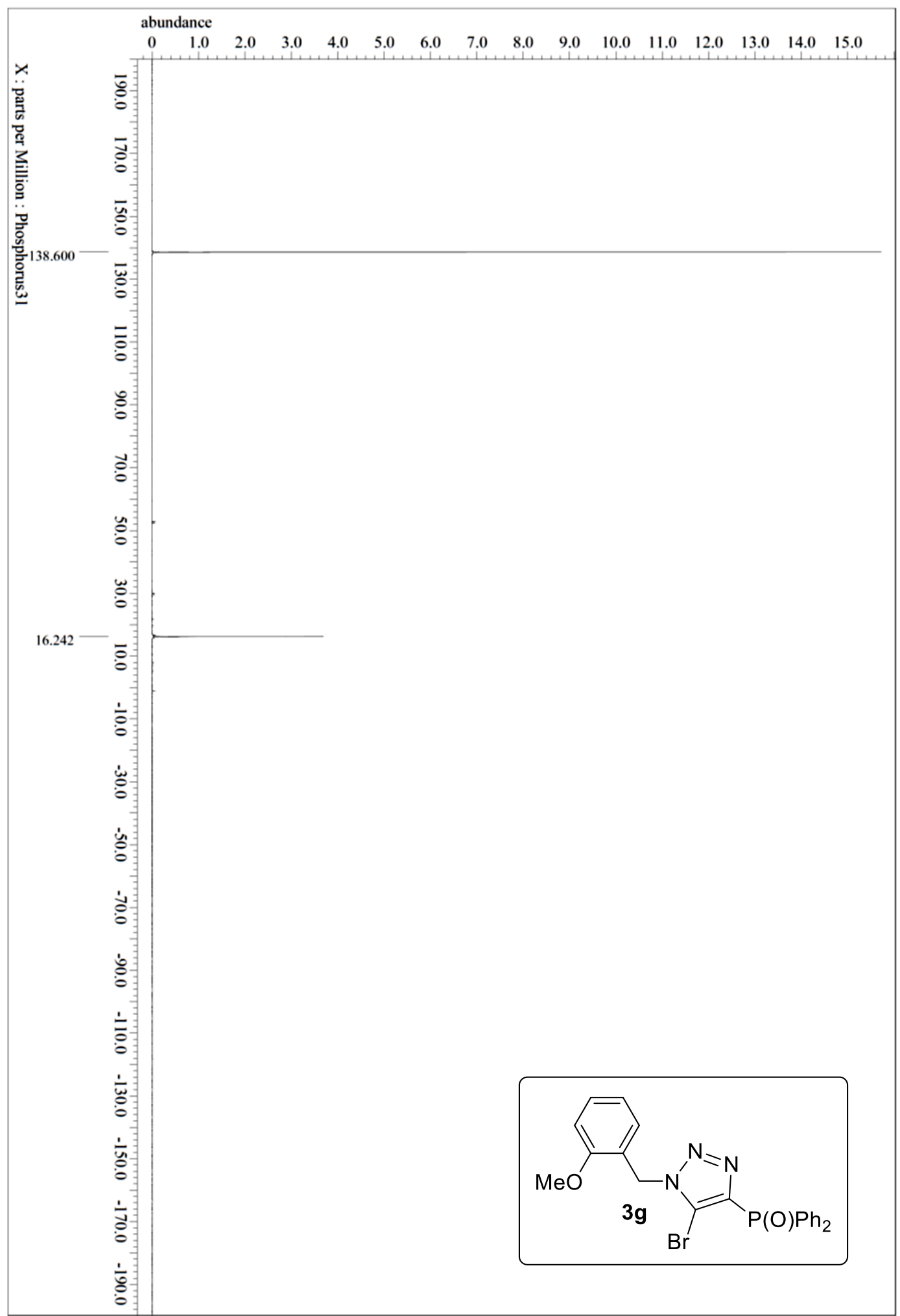

Figure S18. ${ }^{1} \mathrm{H}(400 \mathrm{MHz}),{ }^{13} \mathrm{C}\left\{{ }^{1} \mathrm{H}\right\}$ NMR $(101 \mathrm{MHz})$ and ${ }^{31} \mathrm{P}\left\{{ }^{1} \mathrm{H}\right\}(162 \mathrm{MHz})$ spectra of $\mathbf{3 g}$ $\left(\mathrm{CDCl}_{3}, \mathrm{rt}\right)$ 


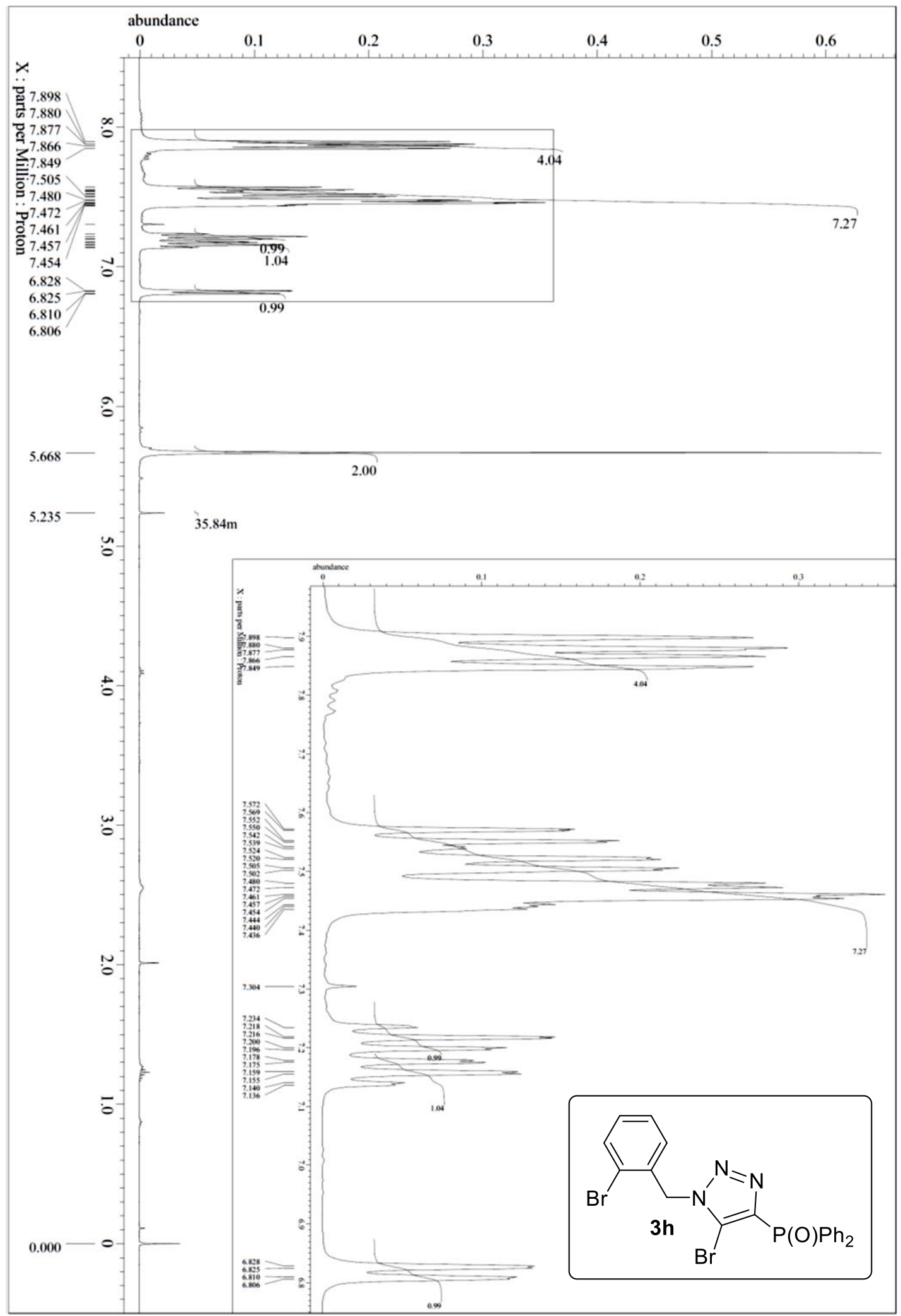




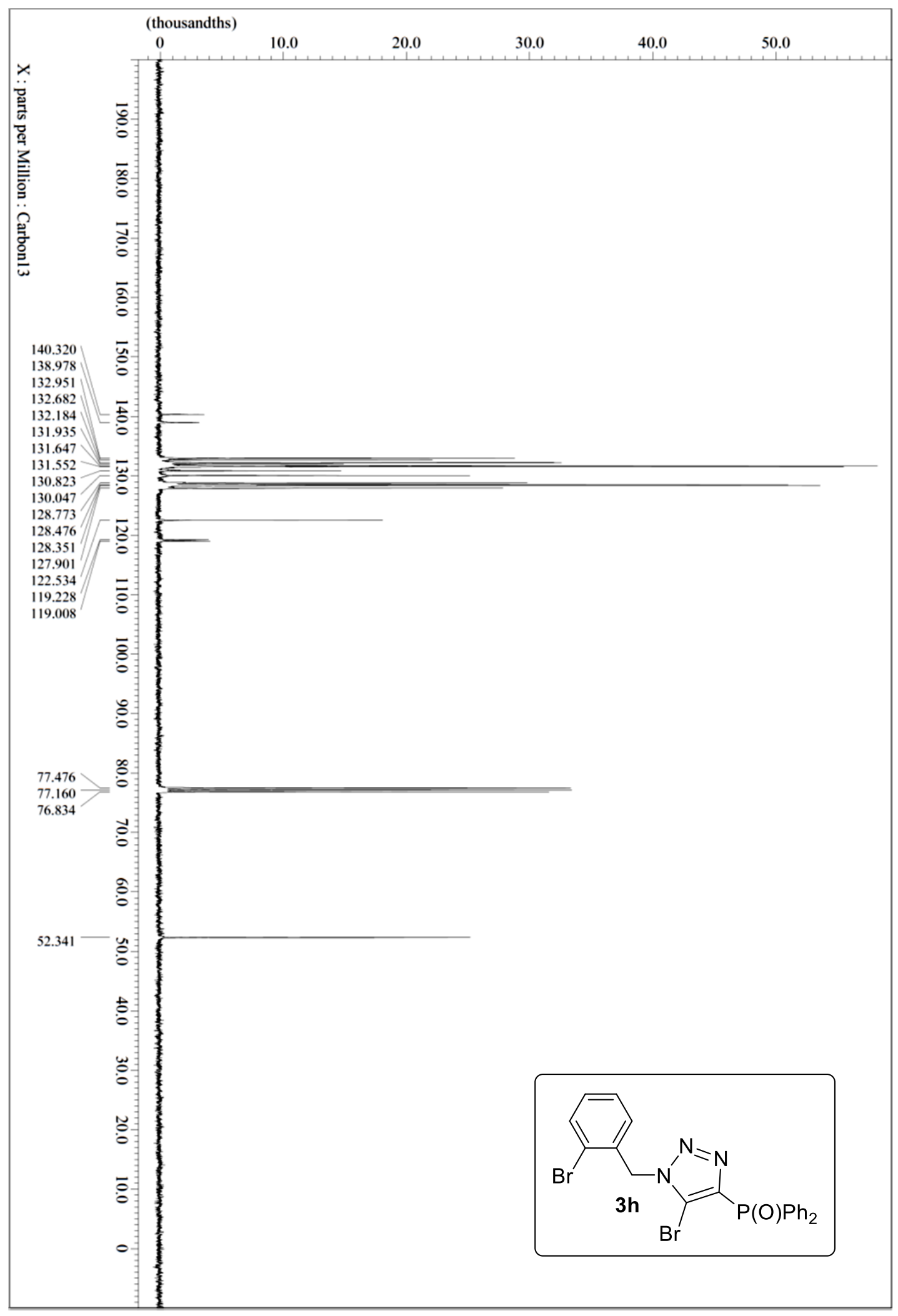




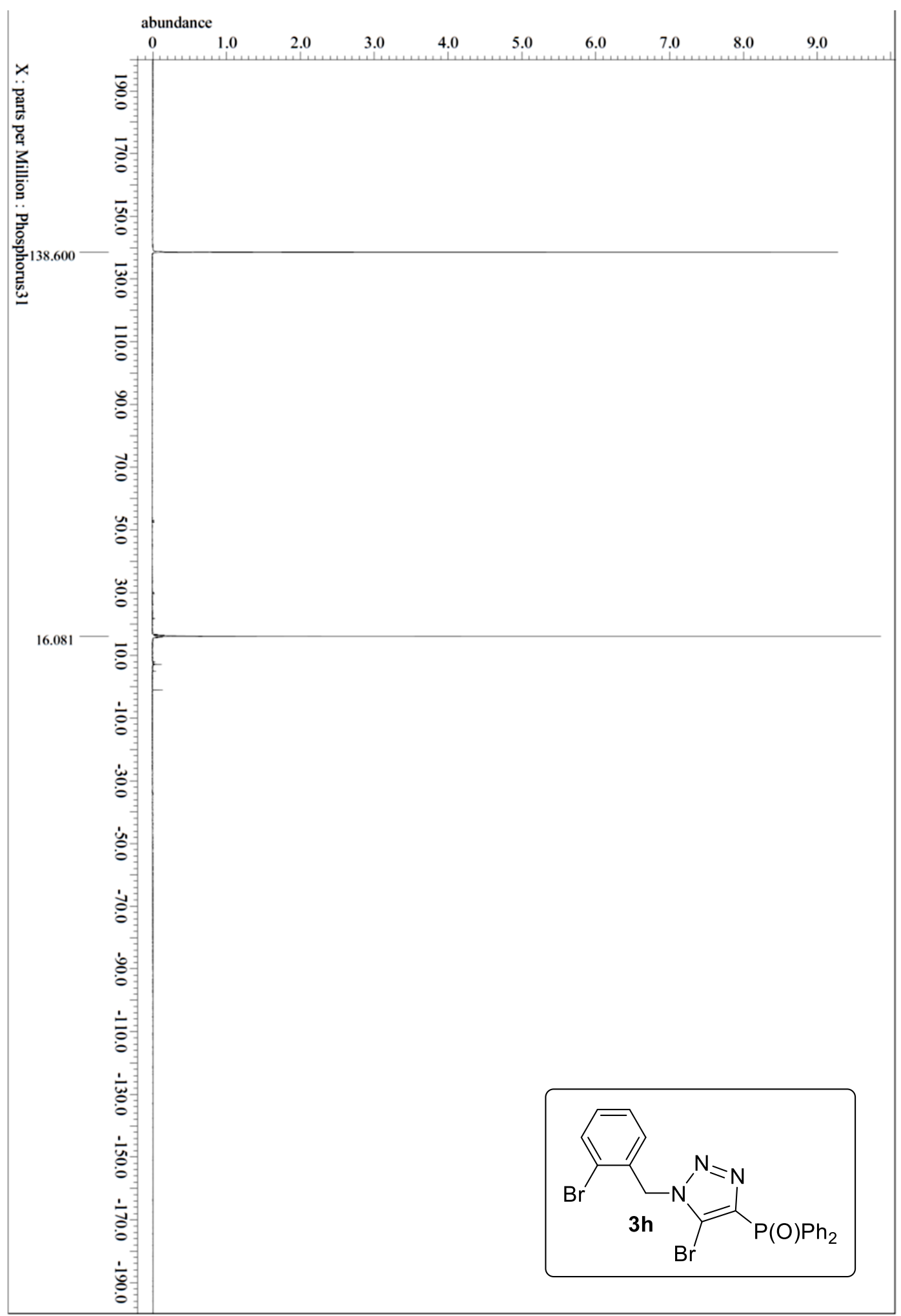

Figure S19. ${ }^{1} \mathrm{H}(400 \mathrm{MHz}),{ }^{13} \mathrm{C}\left\{{ }^{1} \mathrm{H}\right\}$ NMR $(101 \mathrm{MHz})$ and ${ }^{31} \mathrm{P}\left\{{ }^{1} \mathrm{H}\right\}(162 \mathrm{MHz})$ spectra of $\mathbf{3 h}$ $\left(\mathrm{CDCl}_{3}, \mathrm{rt}\right)$ 


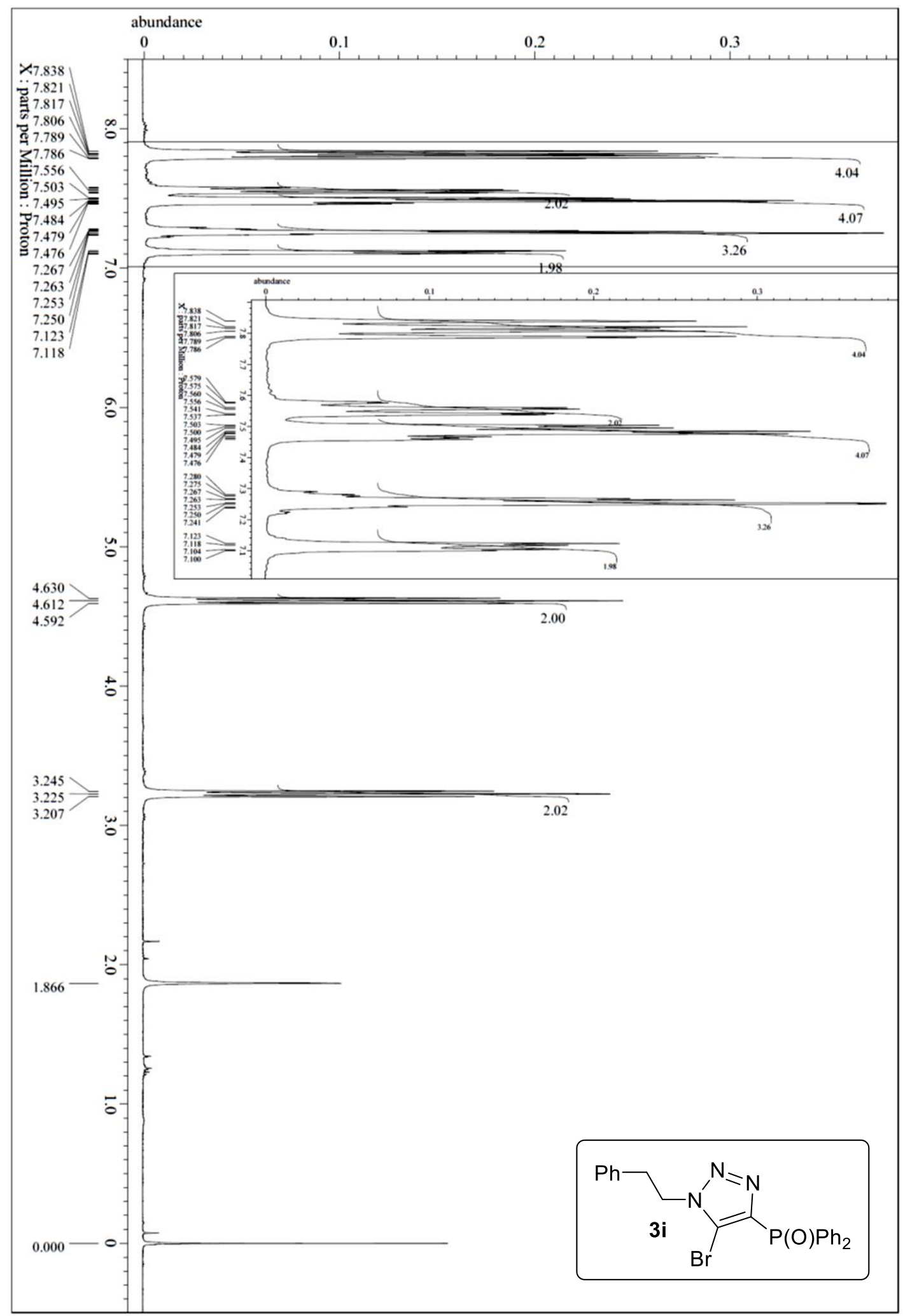




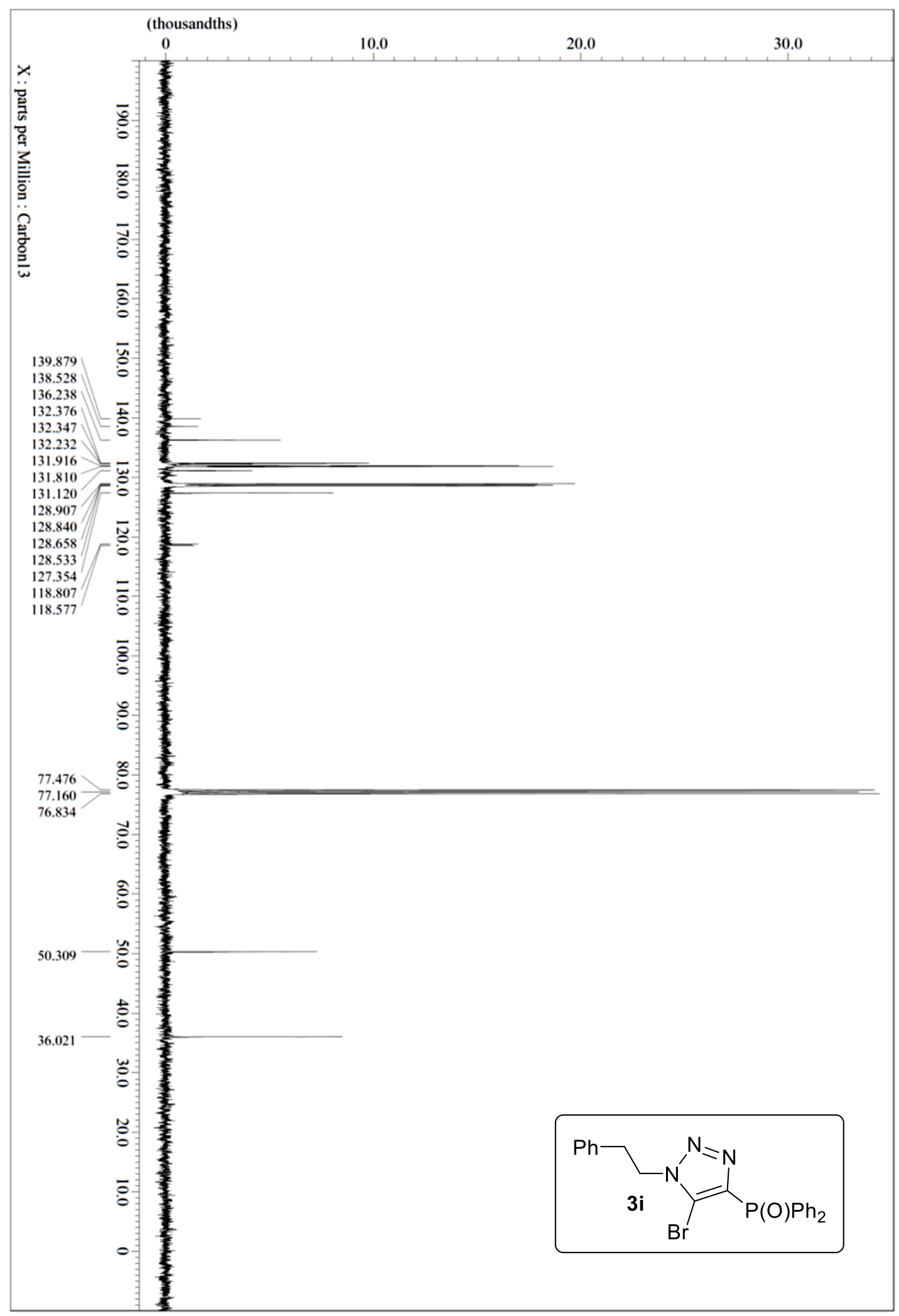




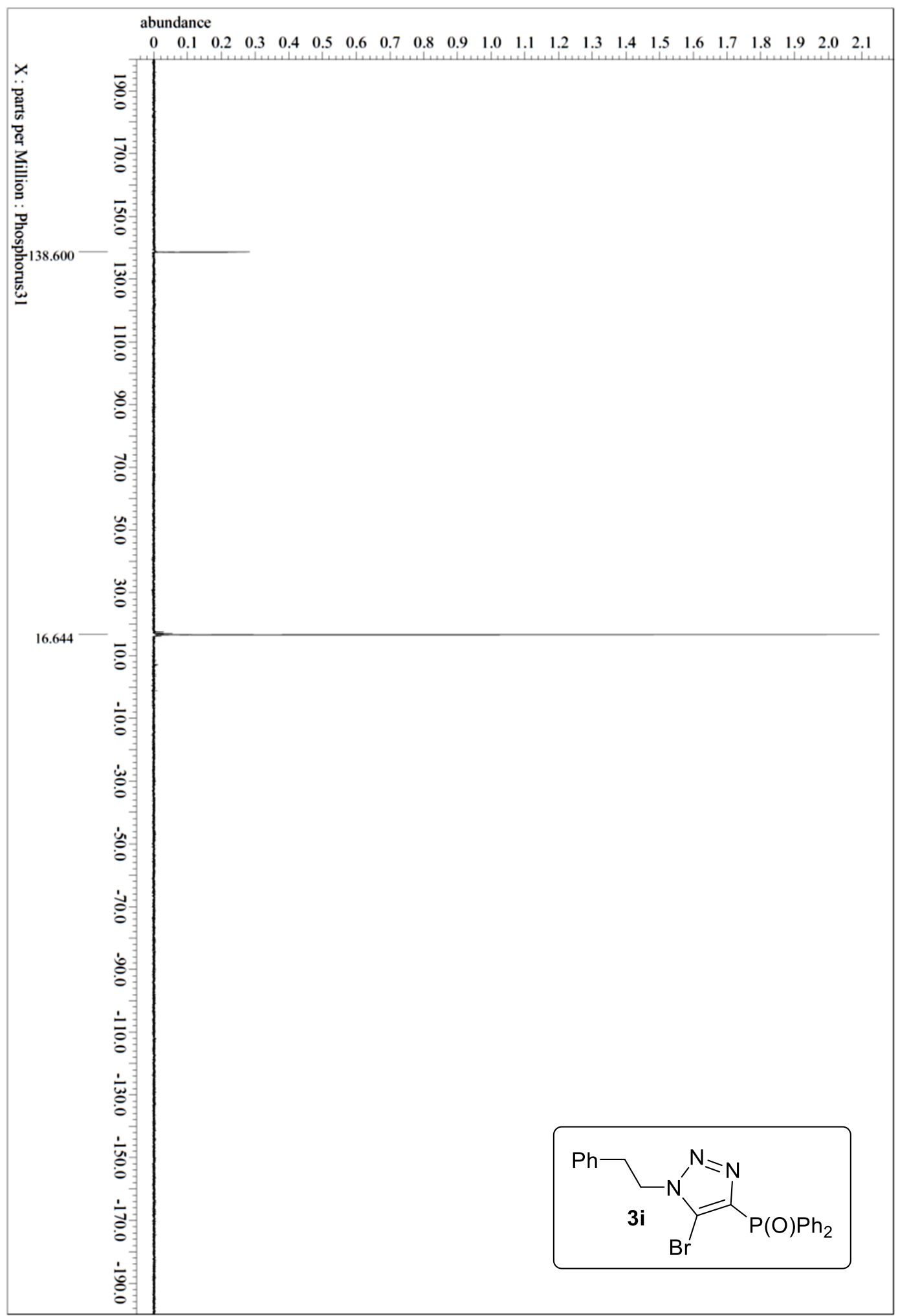

Figure S20. ${ }^{1} \mathrm{H}(400 \mathrm{MHz}),{ }^{13} \mathrm{C}\left\{{ }^{1} \mathrm{H}\right\}$ NMR $(101 \mathrm{MHz})$ and ${ }^{31} \mathrm{P}\left\{{ }^{1} \mathrm{H}\right\}(162 \mathrm{MHz})$ spectra of 3i $\left(\mathrm{CDCl}_{3}, \mathrm{rt}\right)$ 


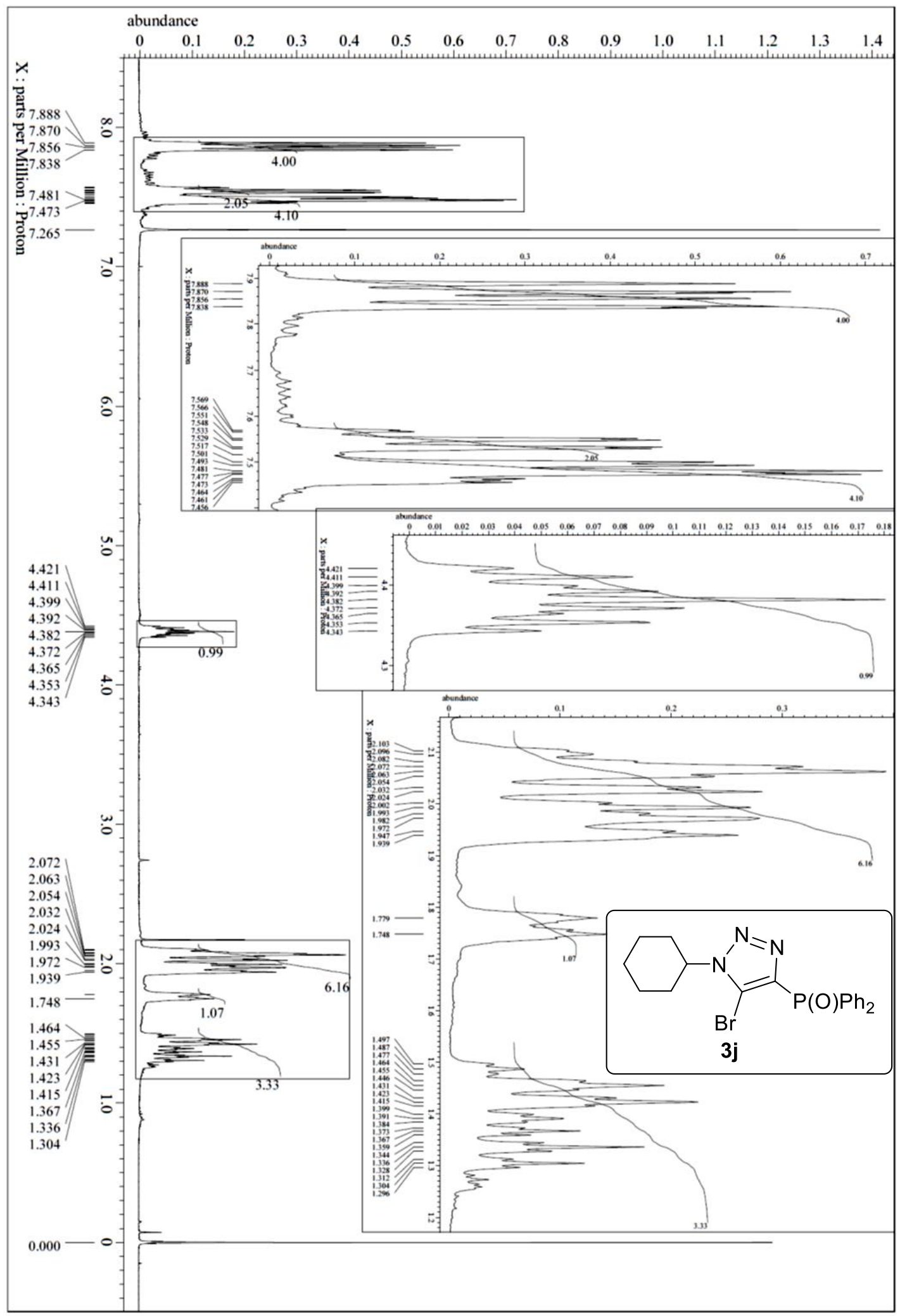




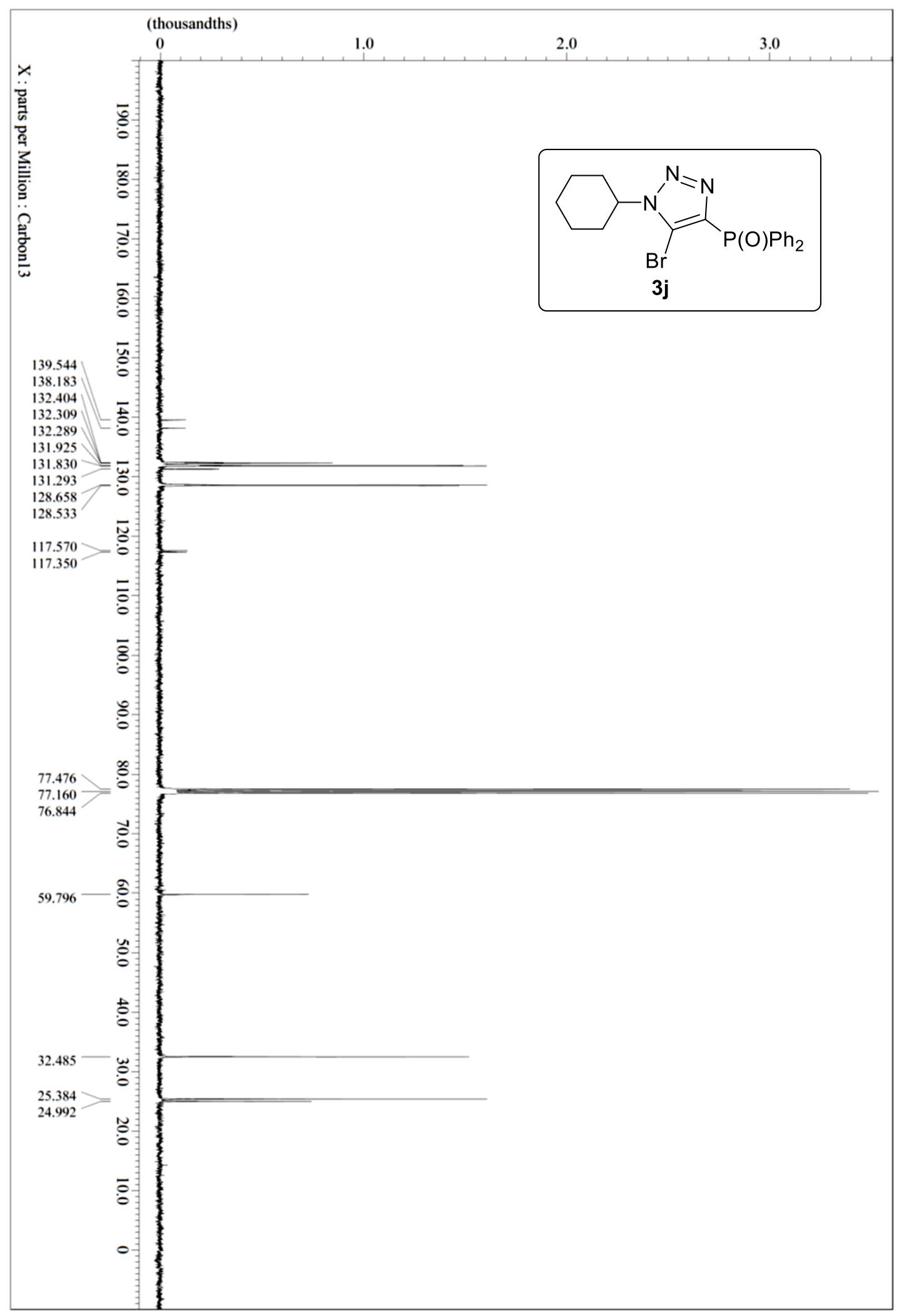




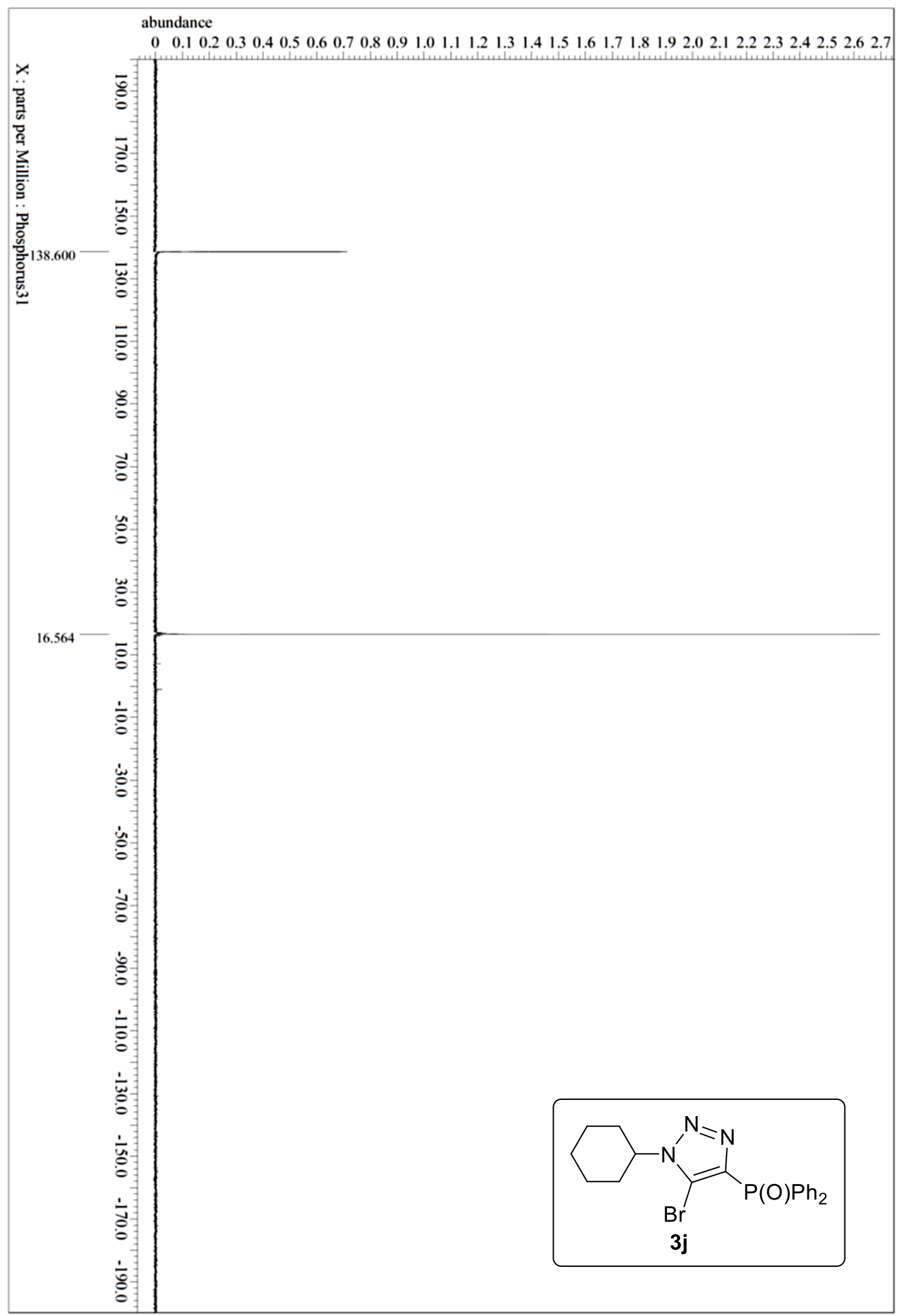

Figure S21. ${ }^{1} \mathrm{H}(400 \mathrm{MHz}),{ }^{13} \mathrm{C}\left\{{ }^{1} \mathrm{H}\right\}$ NMR $(101 \mathrm{MHz})$ and ${ }^{31} \mathrm{P}\left\{{ }^{1} \mathrm{H}\right\}(162 \mathrm{MHz})$ spectra of $\mathbf{3 j}$ $\left(\mathrm{CDCl}_{3}, \mathrm{rt}\right)$ 


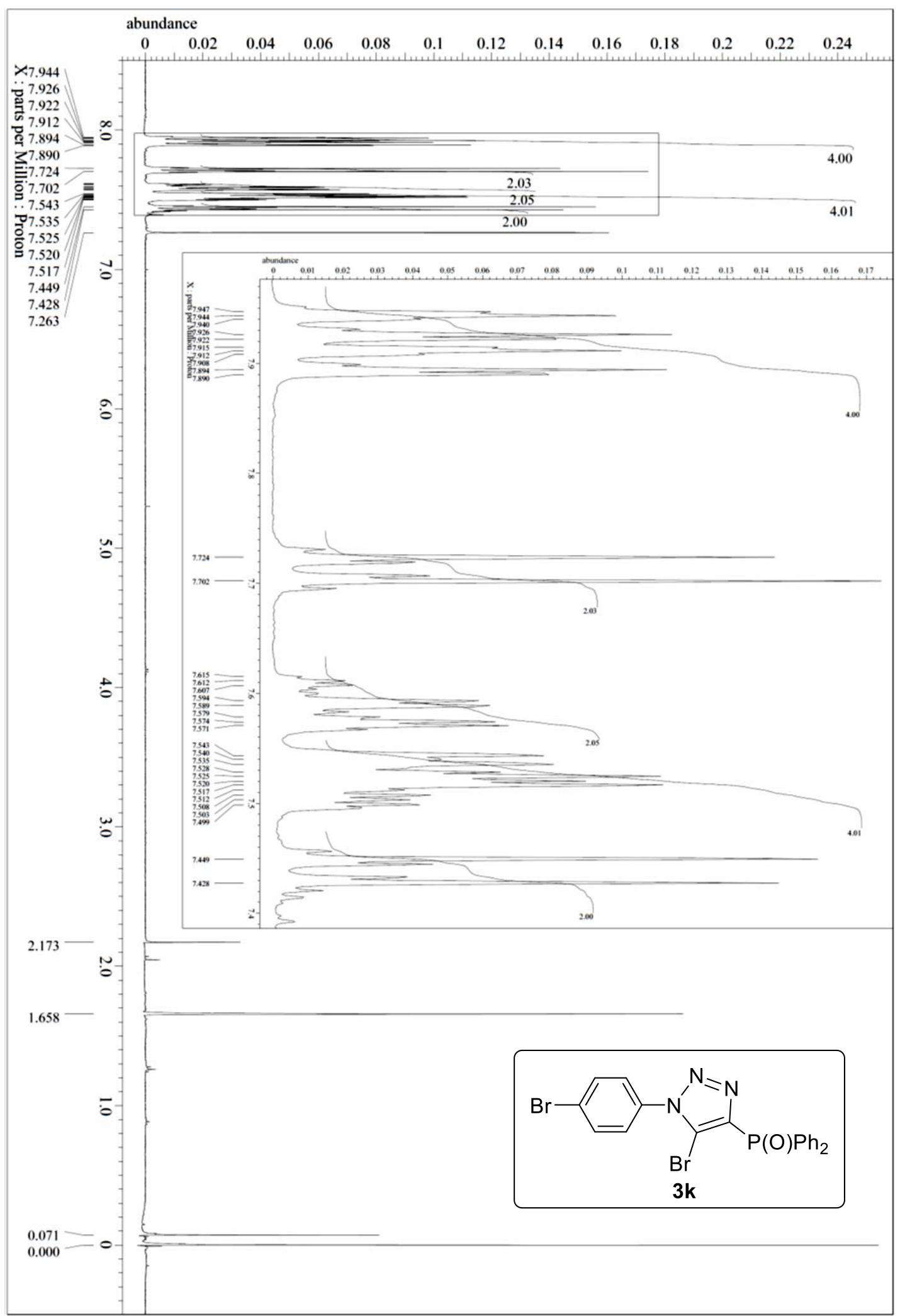




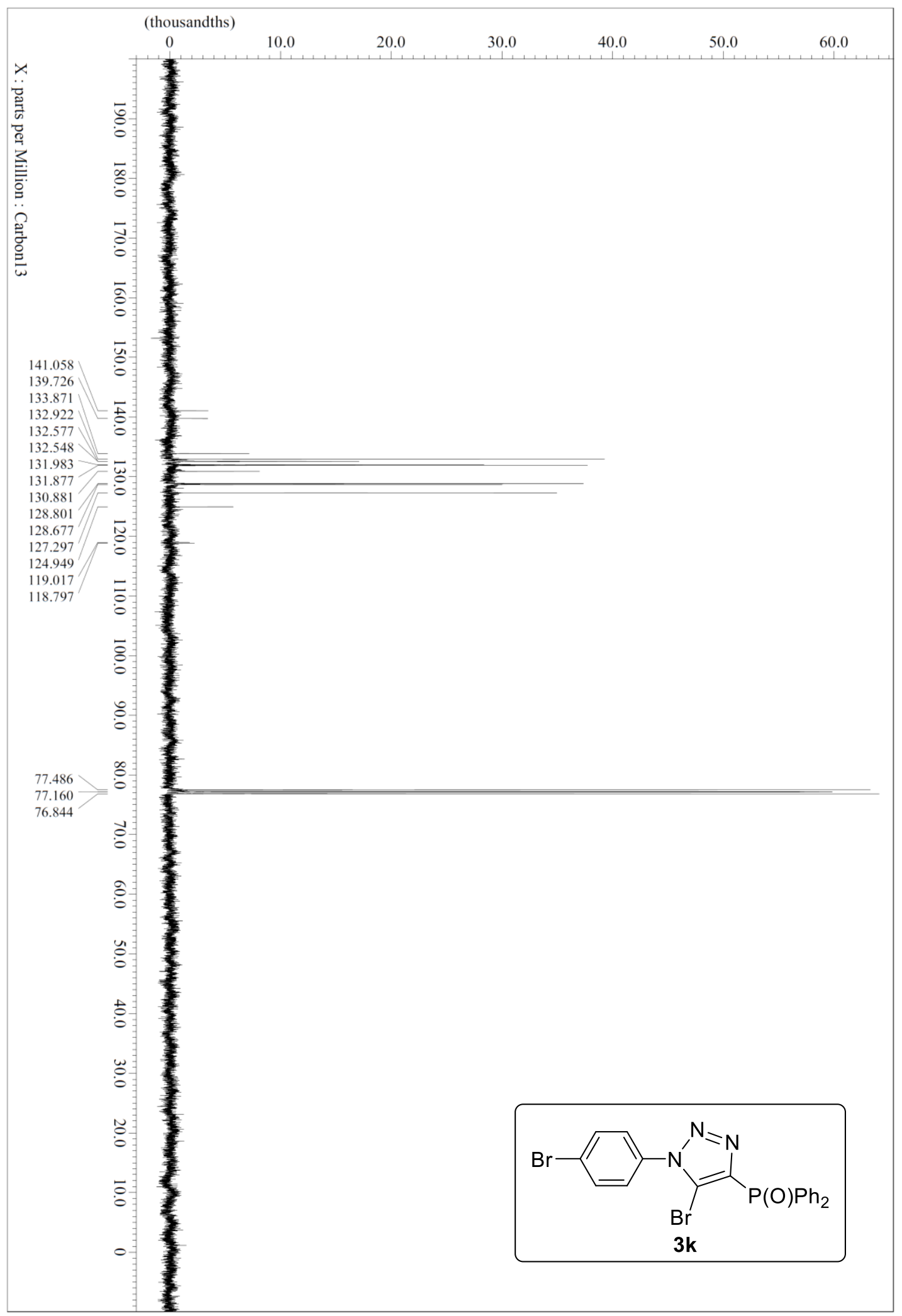




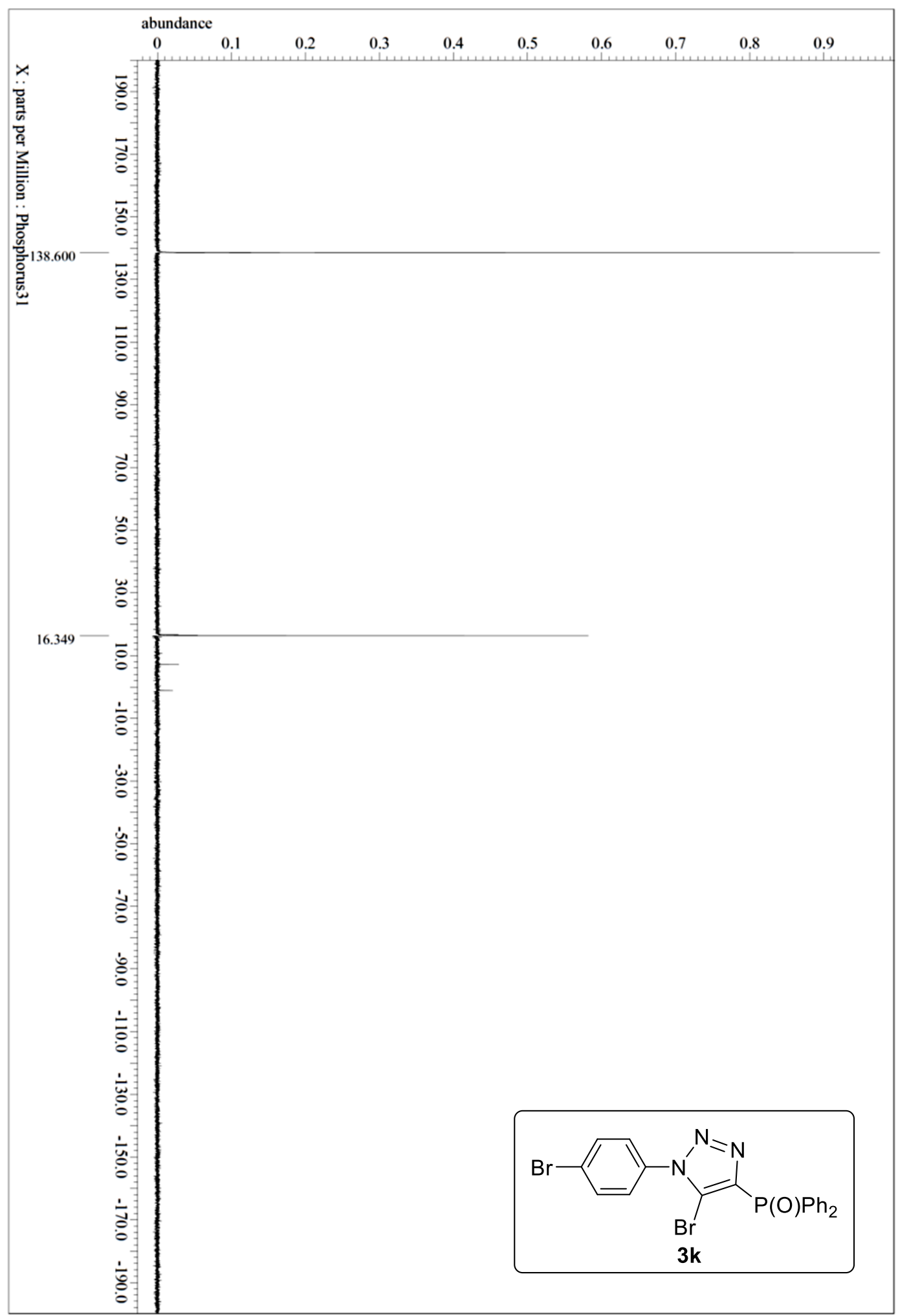

Figure S22. ${ }^{1} \mathrm{H}(400 \mathrm{MHz}),{ }^{13} \mathrm{C}\left\{{ }^{1} \mathrm{H}\right\}$ NMR $(101 \mathrm{MHz})$ and ${ }^{31} \mathrm{P}\left\{{ }^{1} \mathrm{H}\right\}(162 \mathrm{MHz})$ spectra of 3k $\left(\mathrm{CDCl}_{3}, \mathrm{rt}\right)$ 


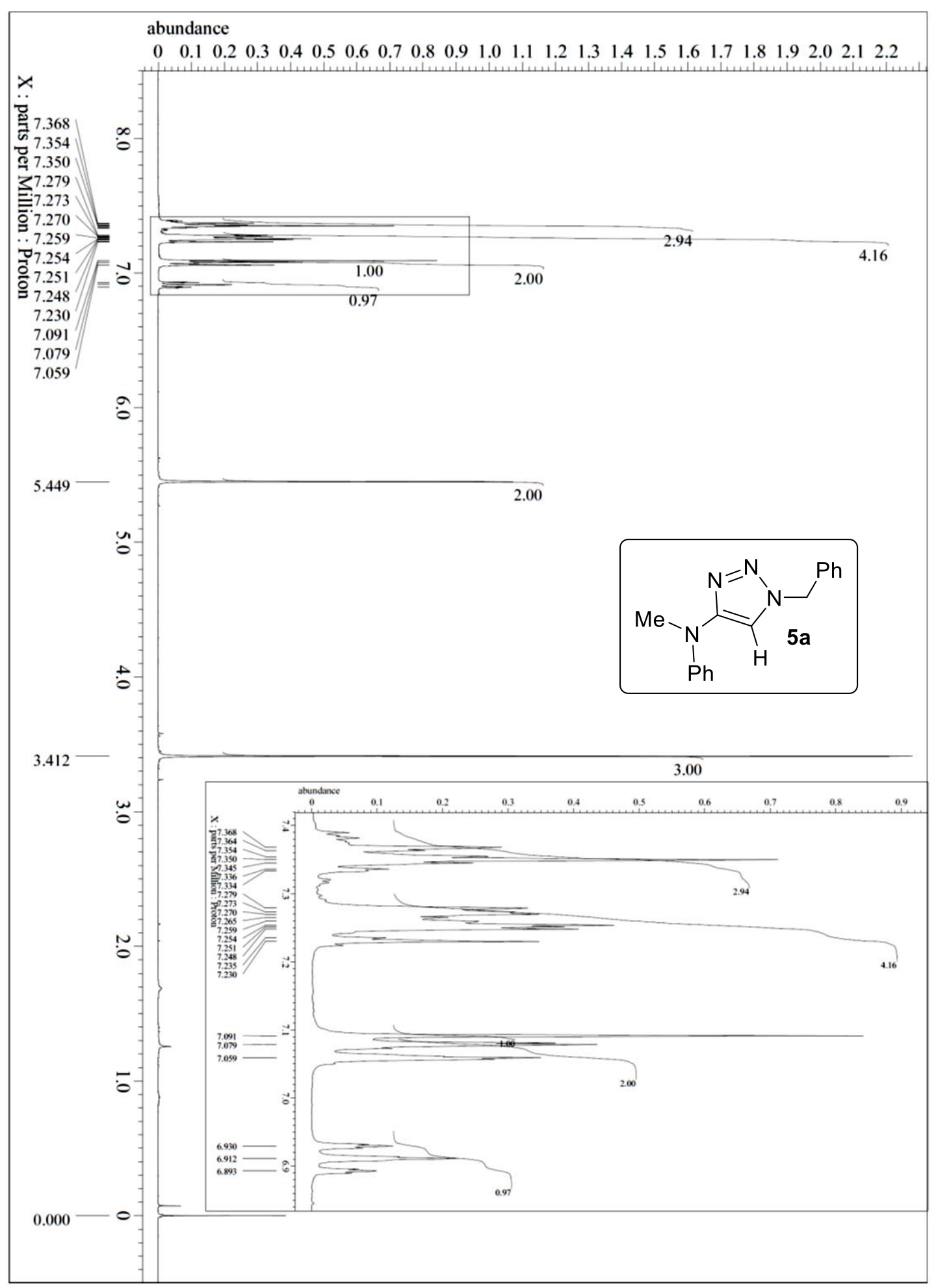




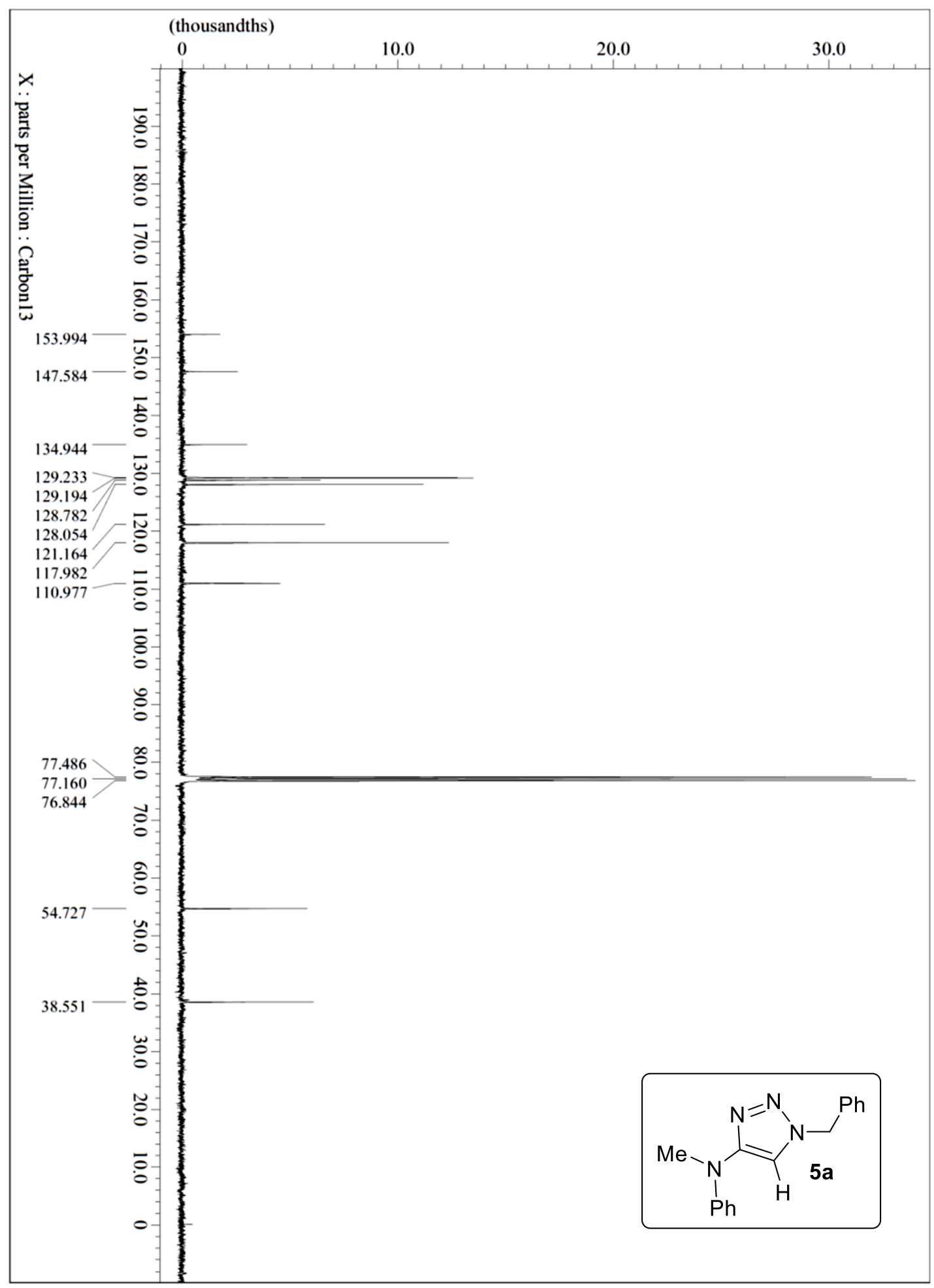

Figure S23. ${ }^{1} \mathrm{H}(400 \mathrm{MHz})$ and ${ }^{13} \mathrm{C}\left\{{ }^{1} \mathrm{H}\right\} \mathrm{NMR}(101 \mathrm{MHz})$ spectra of $\mathbf{5 a}\left(\mathrm{CDCl}_{3}, \mathrm{rt}\right)$ 


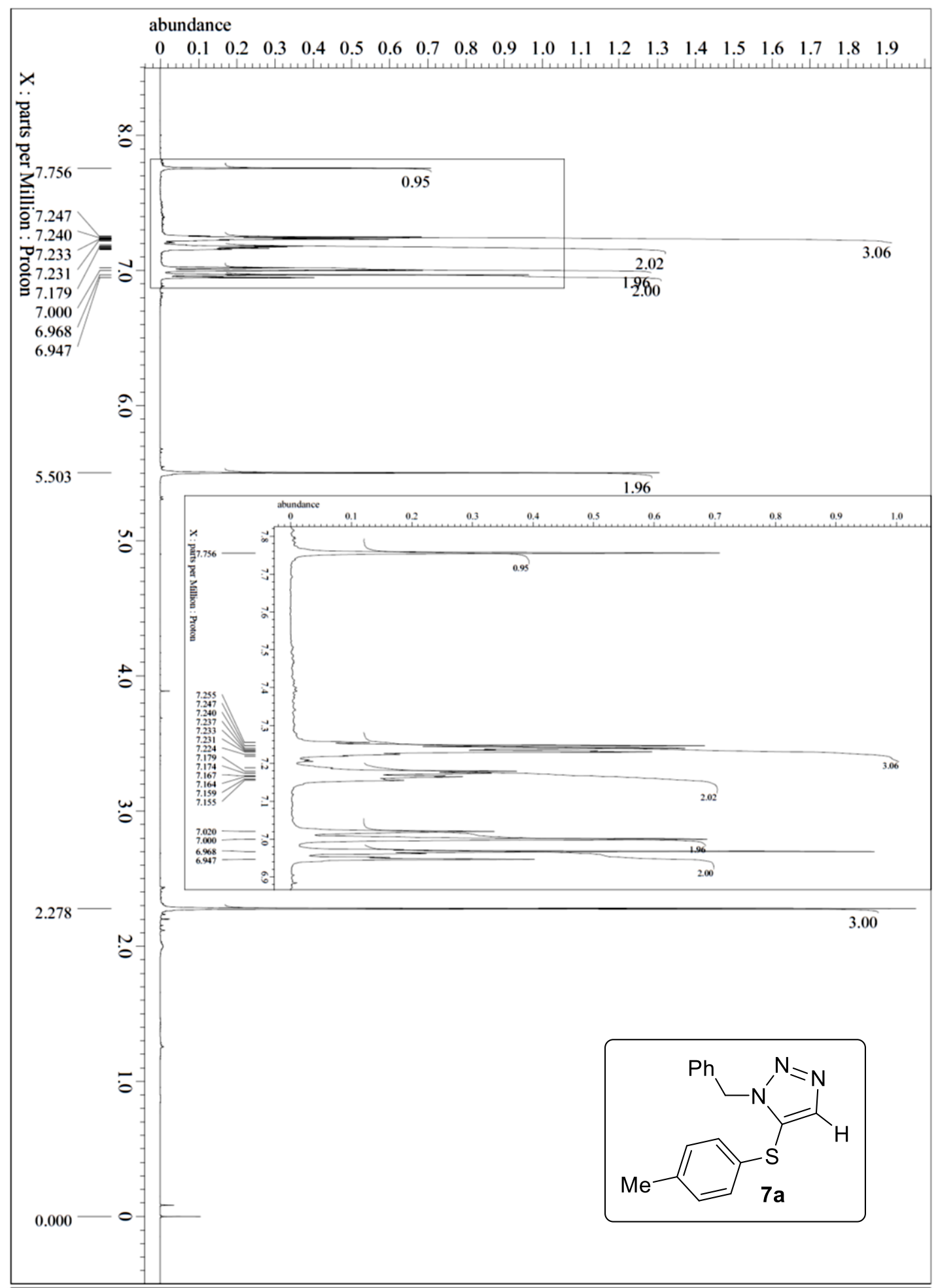




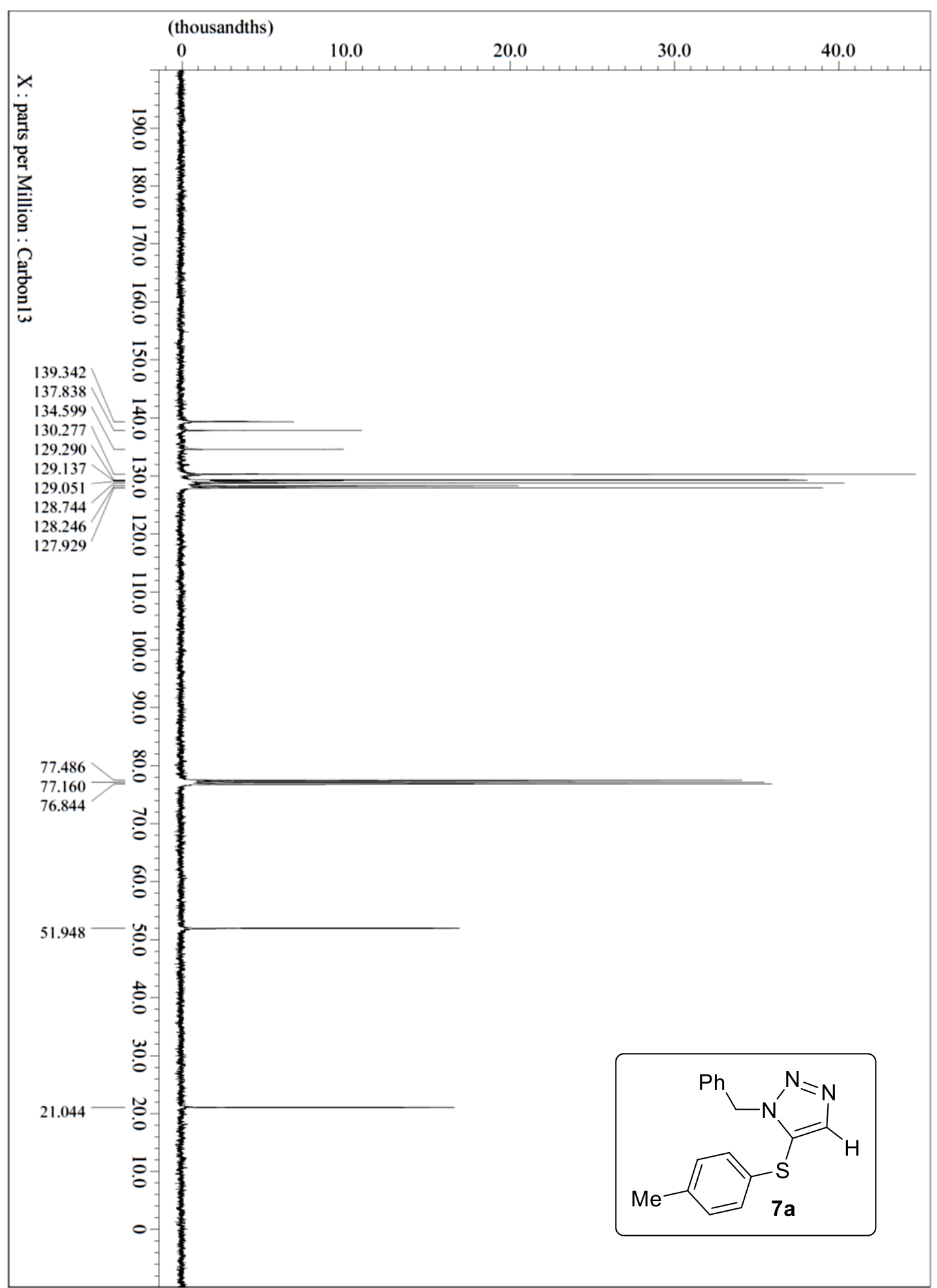

Figure S24. ${ }^{1} \mathrm{H}(400 \mathrm{MHz})$ and ${ }^{13} \mathrm{C}\left\{{ }^{1} \mathrm{H}\right\} \mathrm{NMR}(101 \mathrm{MHz})$ spectra of $7 \mathbf{a}\left(\mathrm{CDCl}_{3}, \mathrm{rt}\right)$ 


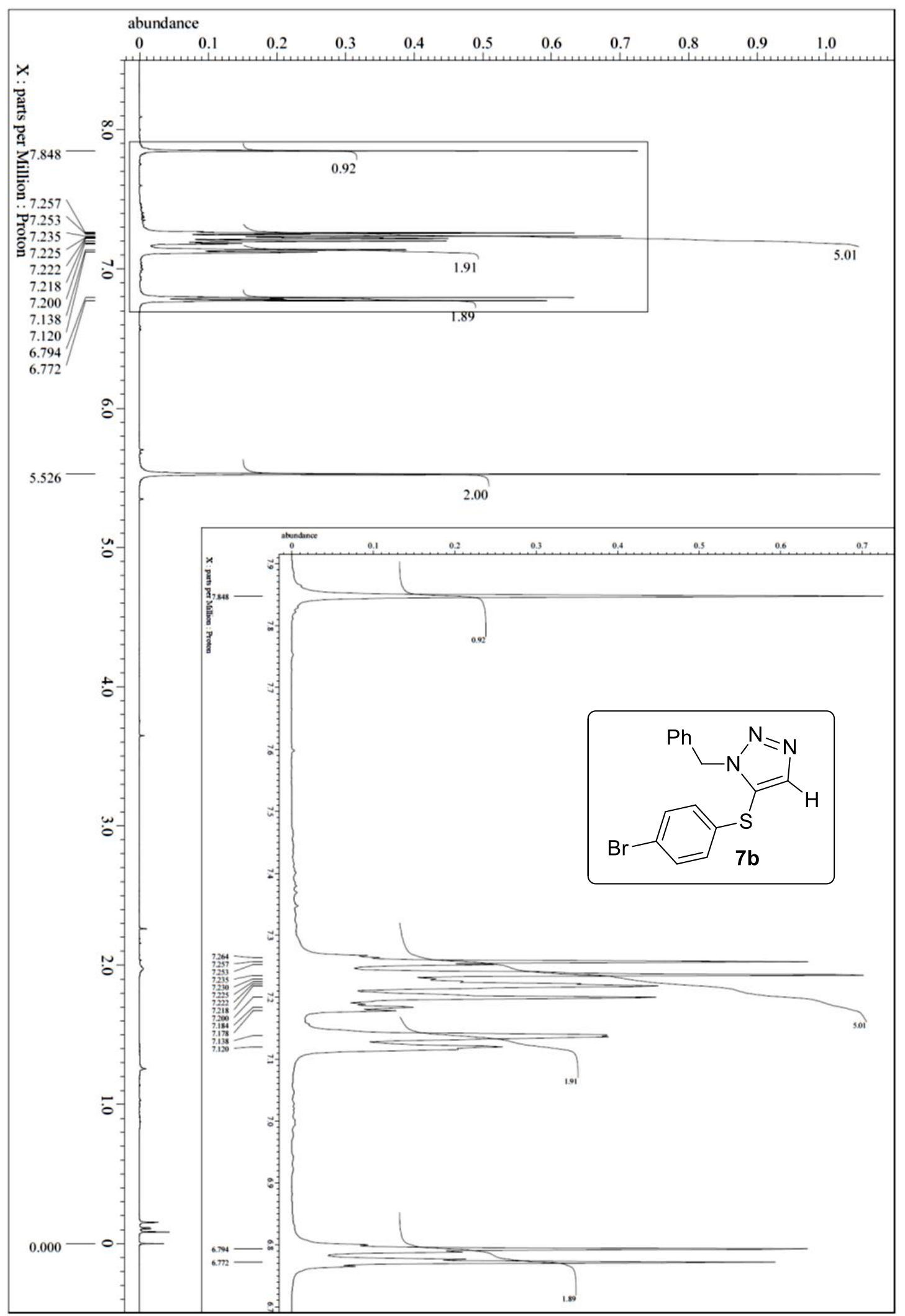




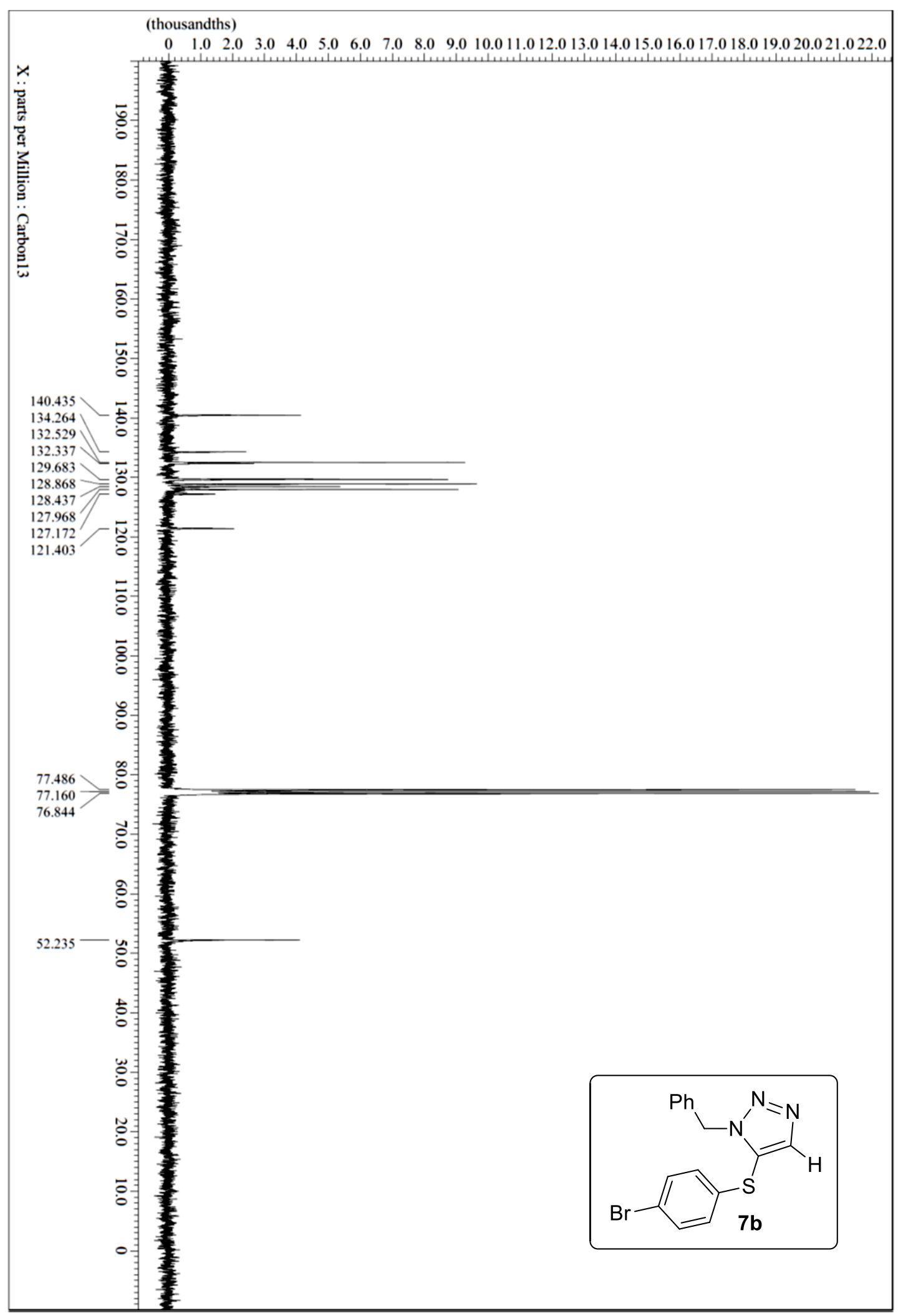

Figure S25. ${ }^{1} \mathrm{H}(400 \mathrm{MHz})$ and ${ }^{13} \mathrm{C}\left\{{ }^{1} \mathrm{H}\right\}$ NMR $(101 \mathrm{MHz})$ spectra of $\mathbf{7 b}\left(\mathrm{CDCl}_{3}, \mathrm{rt}\right)$ 


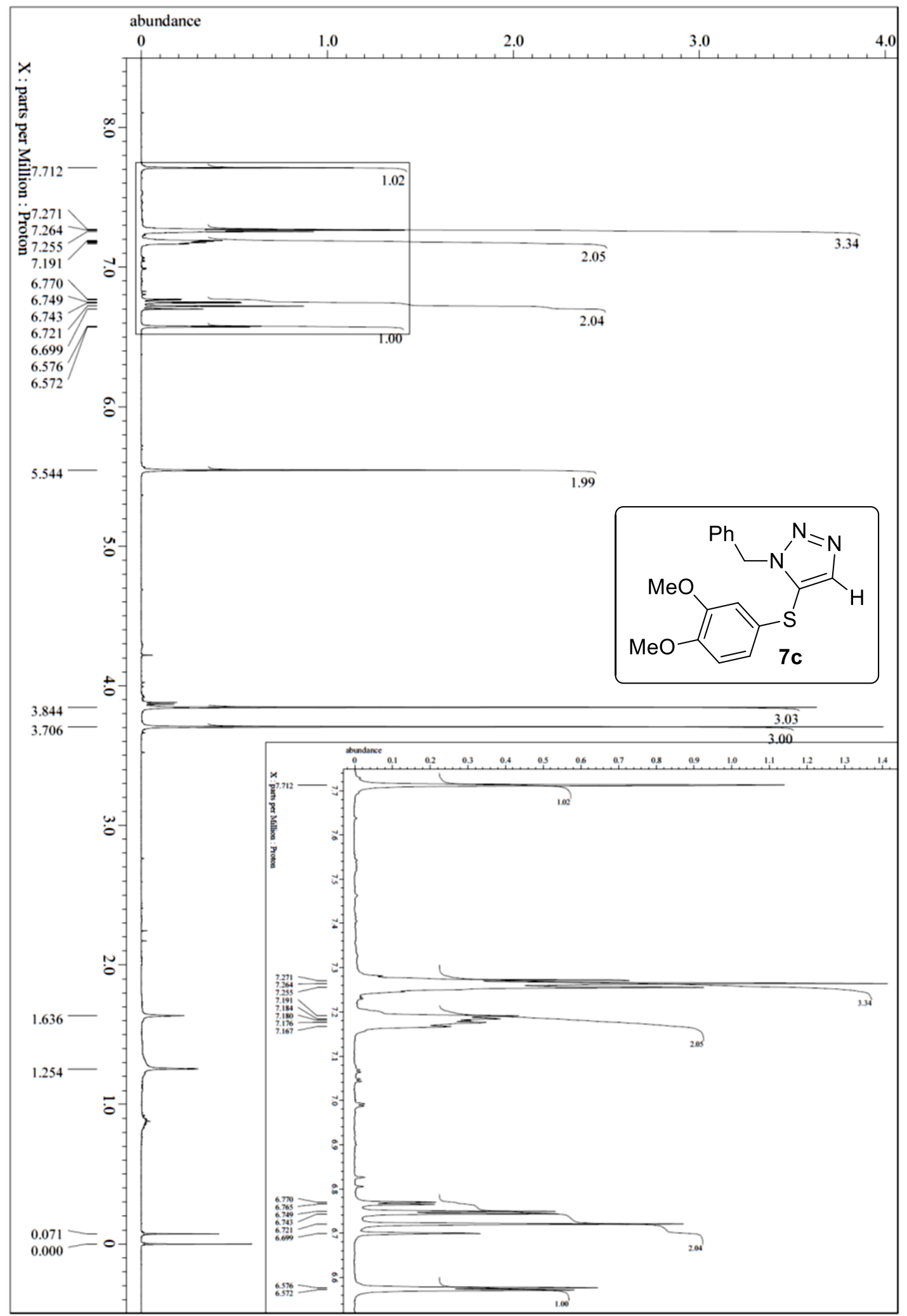




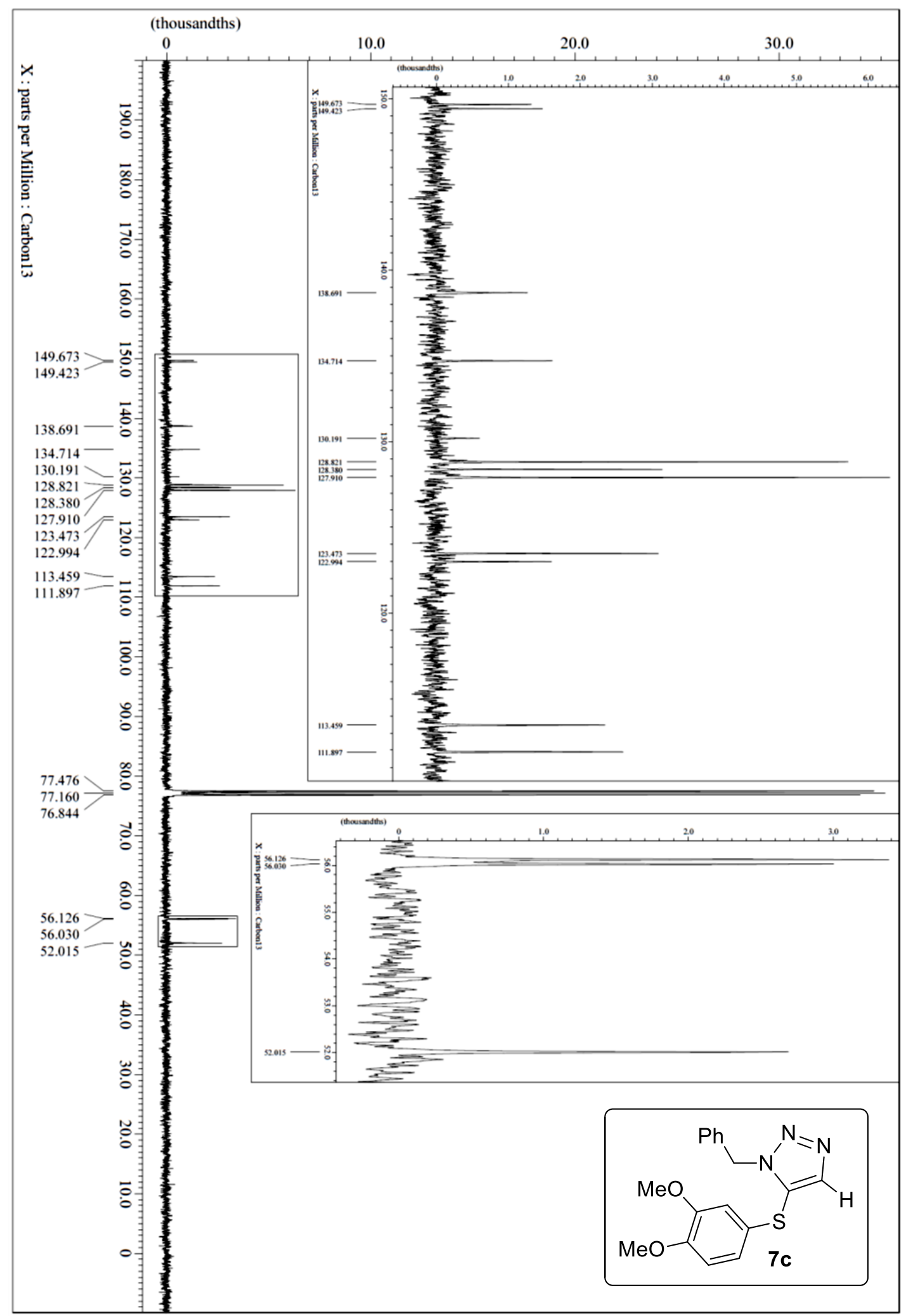

Figure S26. ${ }^{1} \mathrm{H}(400 \mathrm{MHz})$ and ${ }^{13} \mathrm{C}\left\{{ }^{1} \mathrm{H}\right\} \mathrm{NMR}(101 \mathrm{MHz})$ spectra of $7 \mathbf{c}\left(\mathrm{CDCl}_{3}, \mathrm{rt}\right)$ 


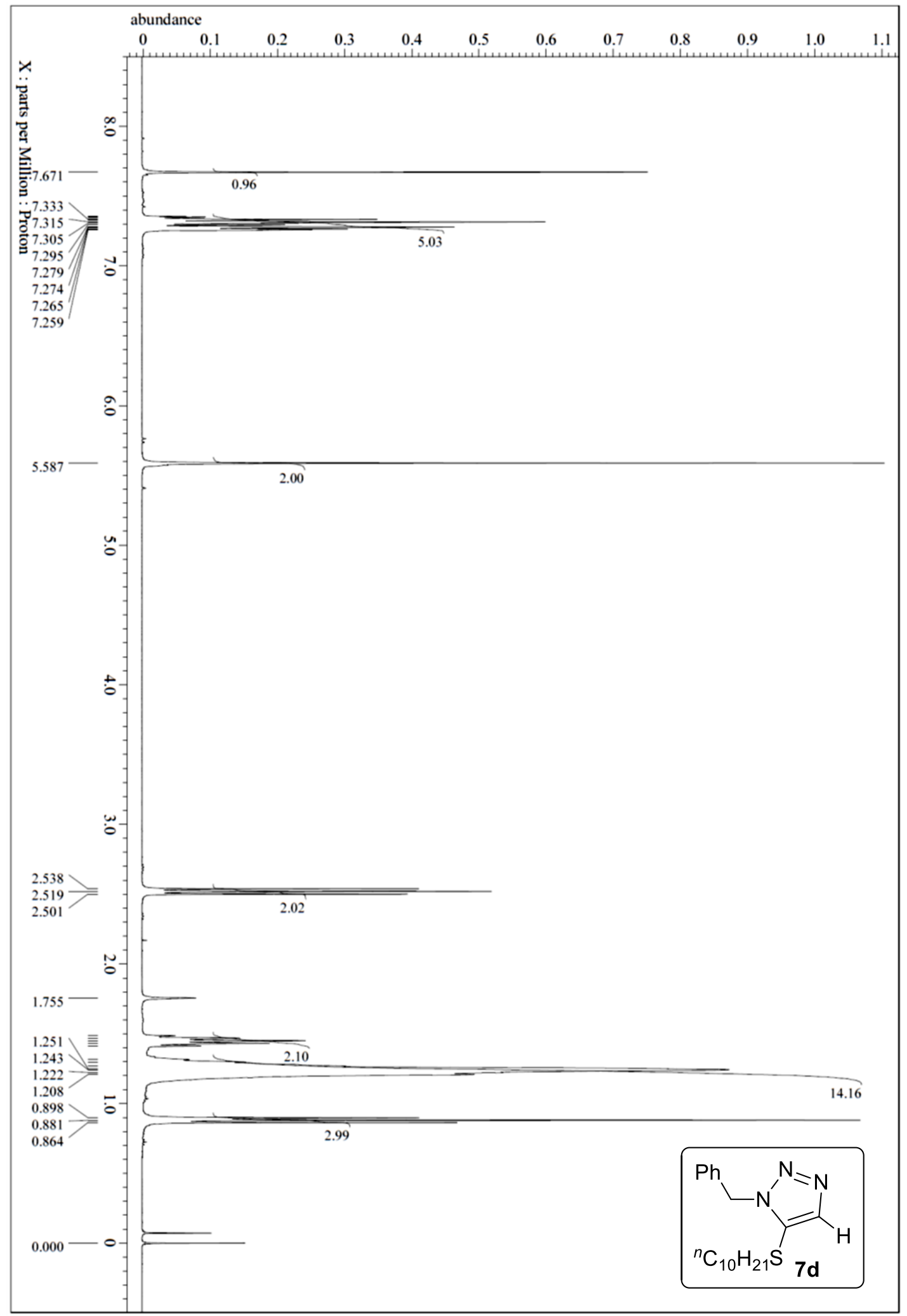




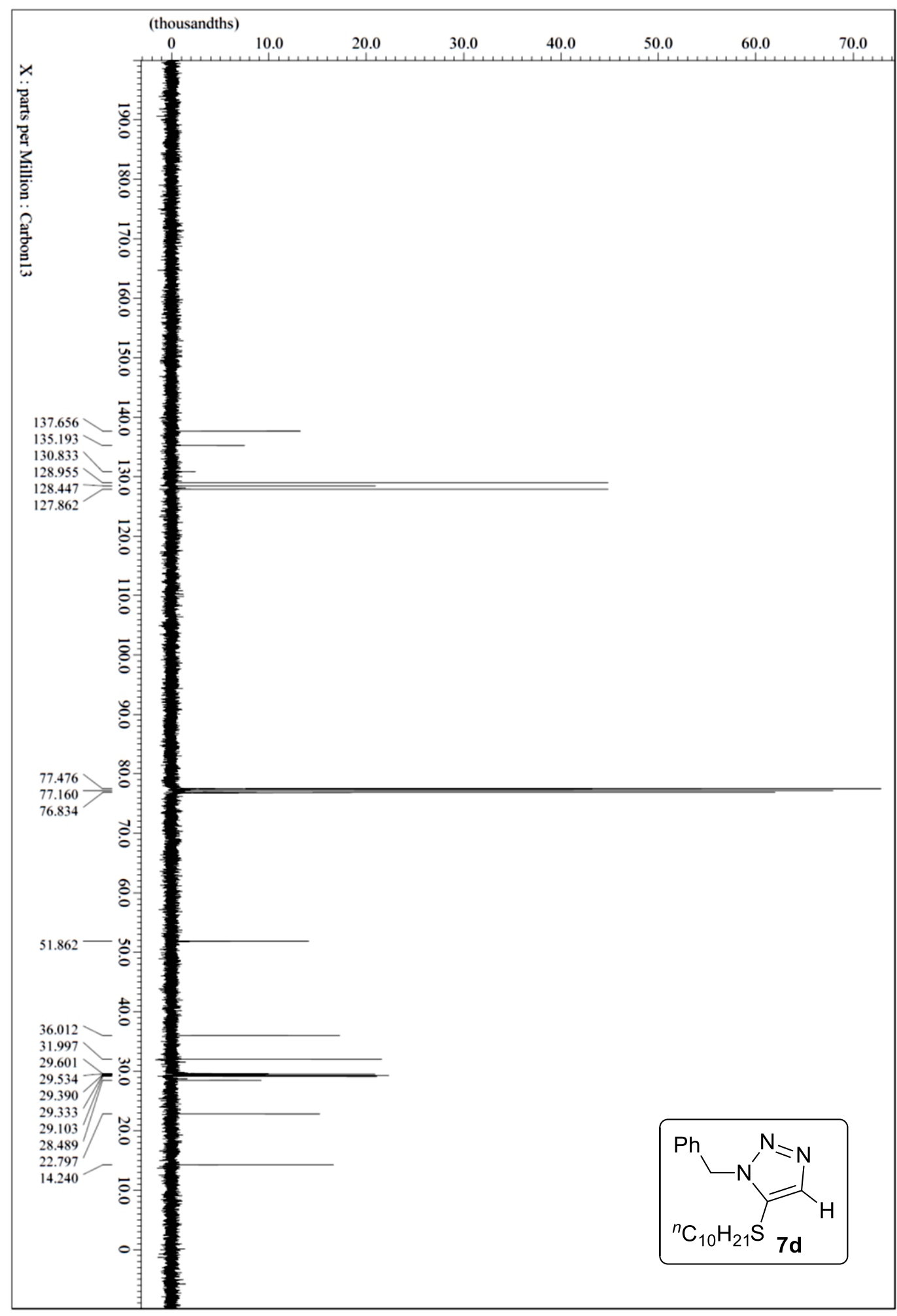

Figure S27. ${ }^{1} \mathrm{H}(400 \mathrm{MHz})$ and ${ }^{13} \mathrm{C}\left\{{ }^{1} \mathrm{H}\right\}$ NMR (101 MHz) spectra of 7d $\left(\mathrm{CDCl}_{3}\right.$, rt) 


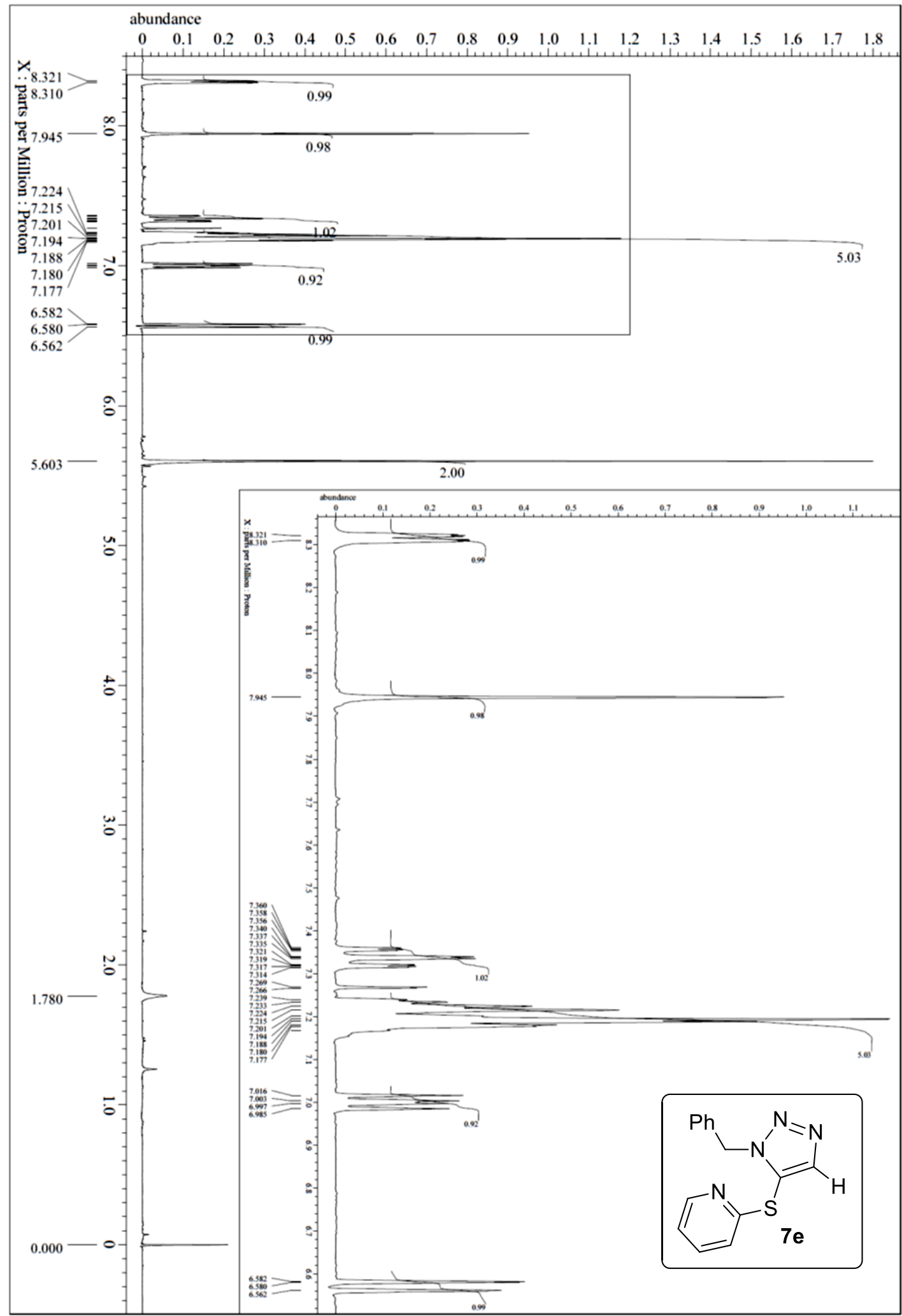




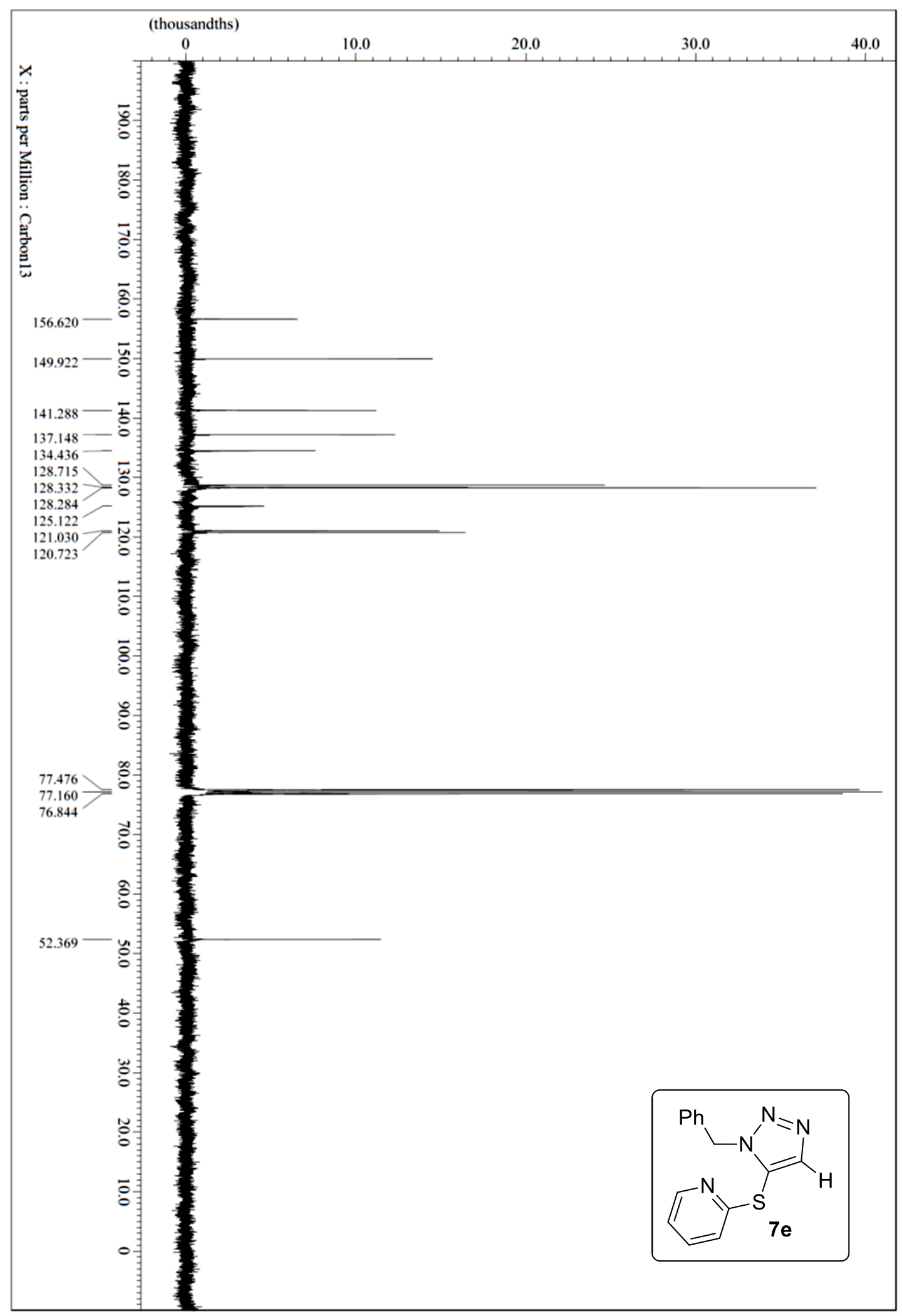

Figure S28. ${ }^{1} \mathrm{H}(400 \mathrm{MHz})$ and ${ }^{13} \mathrm{C}\left\{{ }^{1} \mathrm{H}\right\} \mathrm{NMR}(101 \mathrm{MHz})$ spectra of $7 \mathbf{e}\left(\mathrm{CDCl}_{3}, \mathrm{rt}\right)$ 


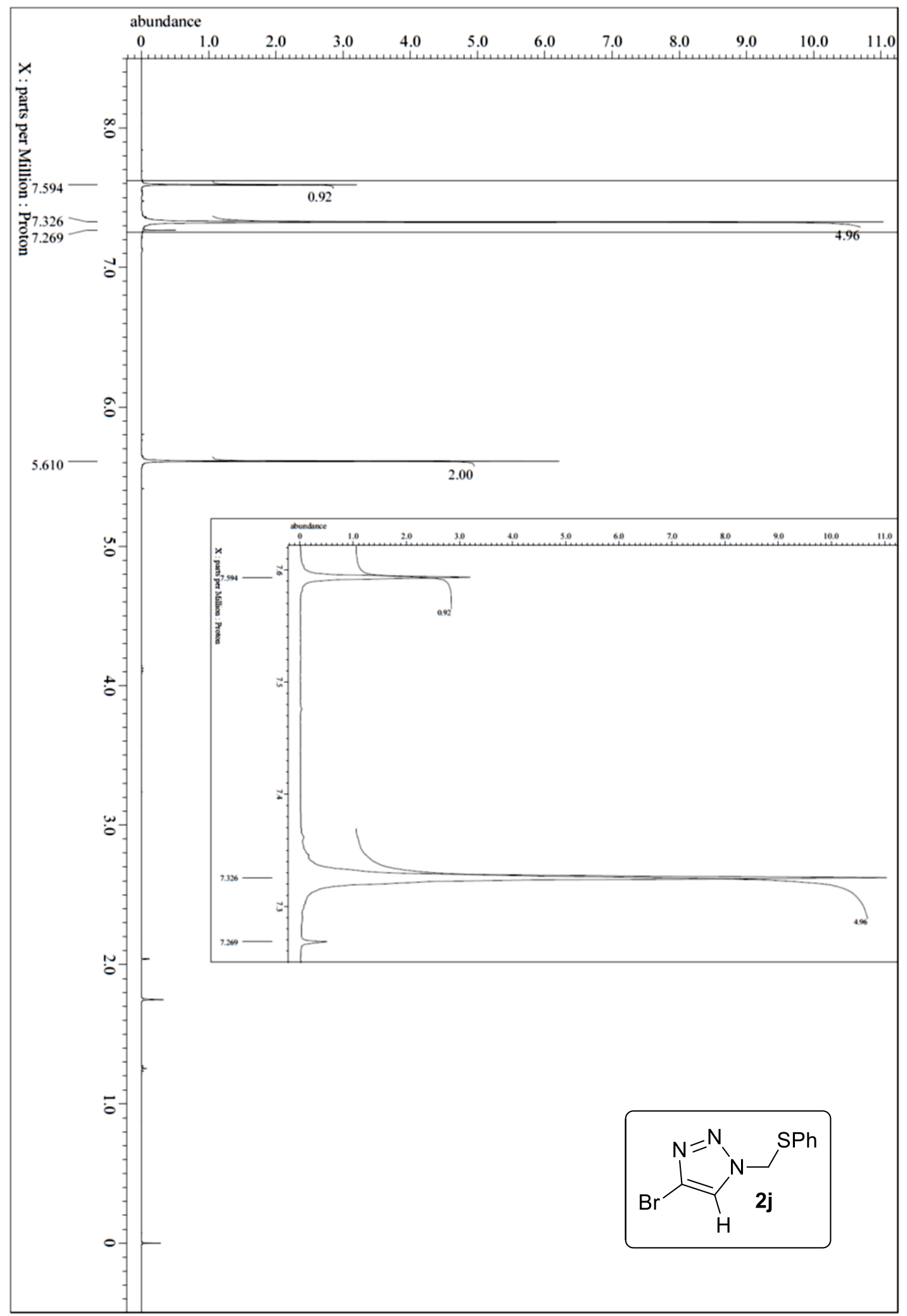




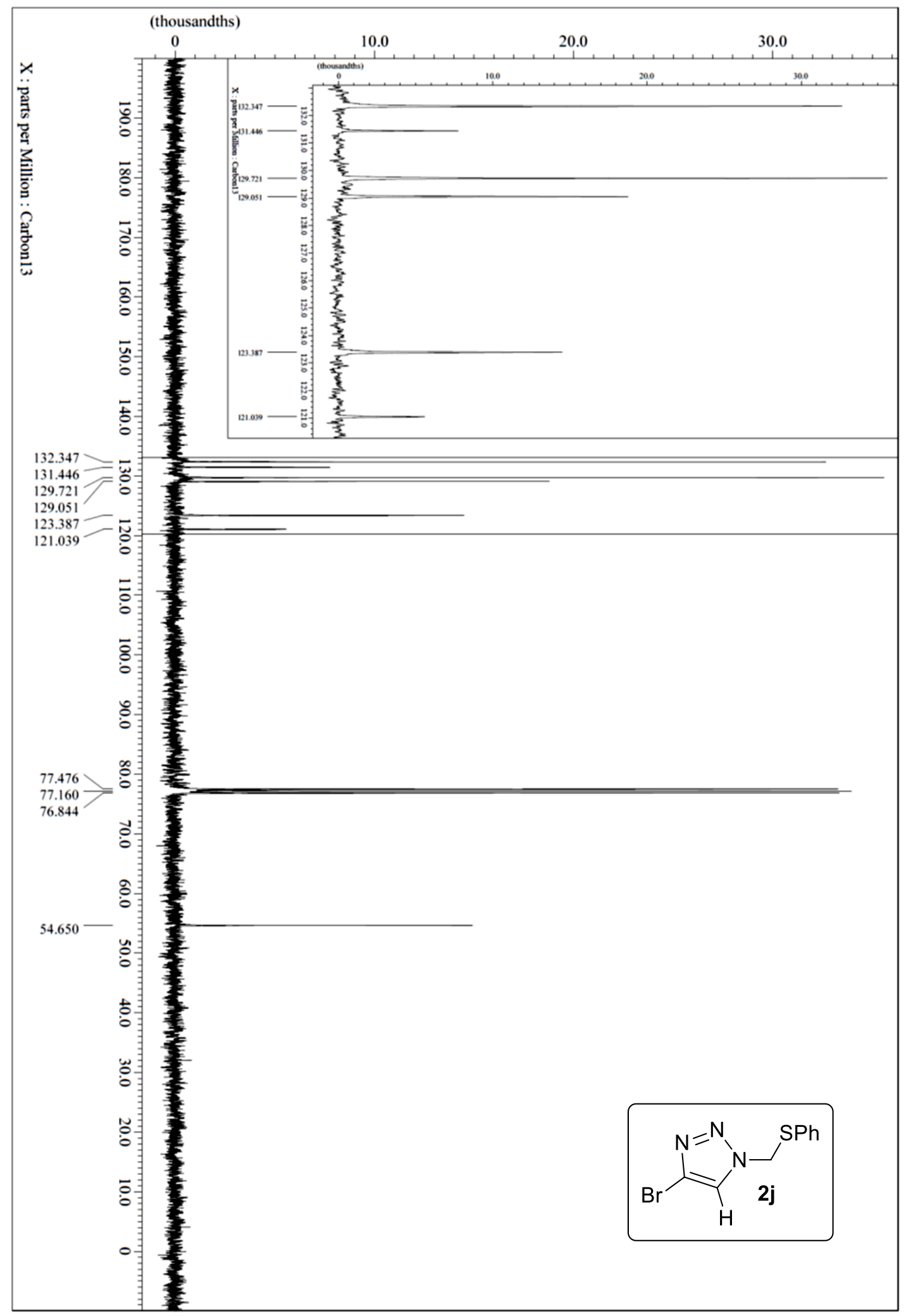

Figure S29. ${ }^{1} \mathrm{H}(400 \mathrm{MHz})$ and ${ }^{13} \mathrm{C}\left\{{ }^{1} \mathrm{H}\right\} \mathrm{NMR}(101 \mathrm{MHz})$ spectra of $\mathbf{2 j}\left(\mathrm{CDCl}_{3}, \mathrm{rt}\right)$ 


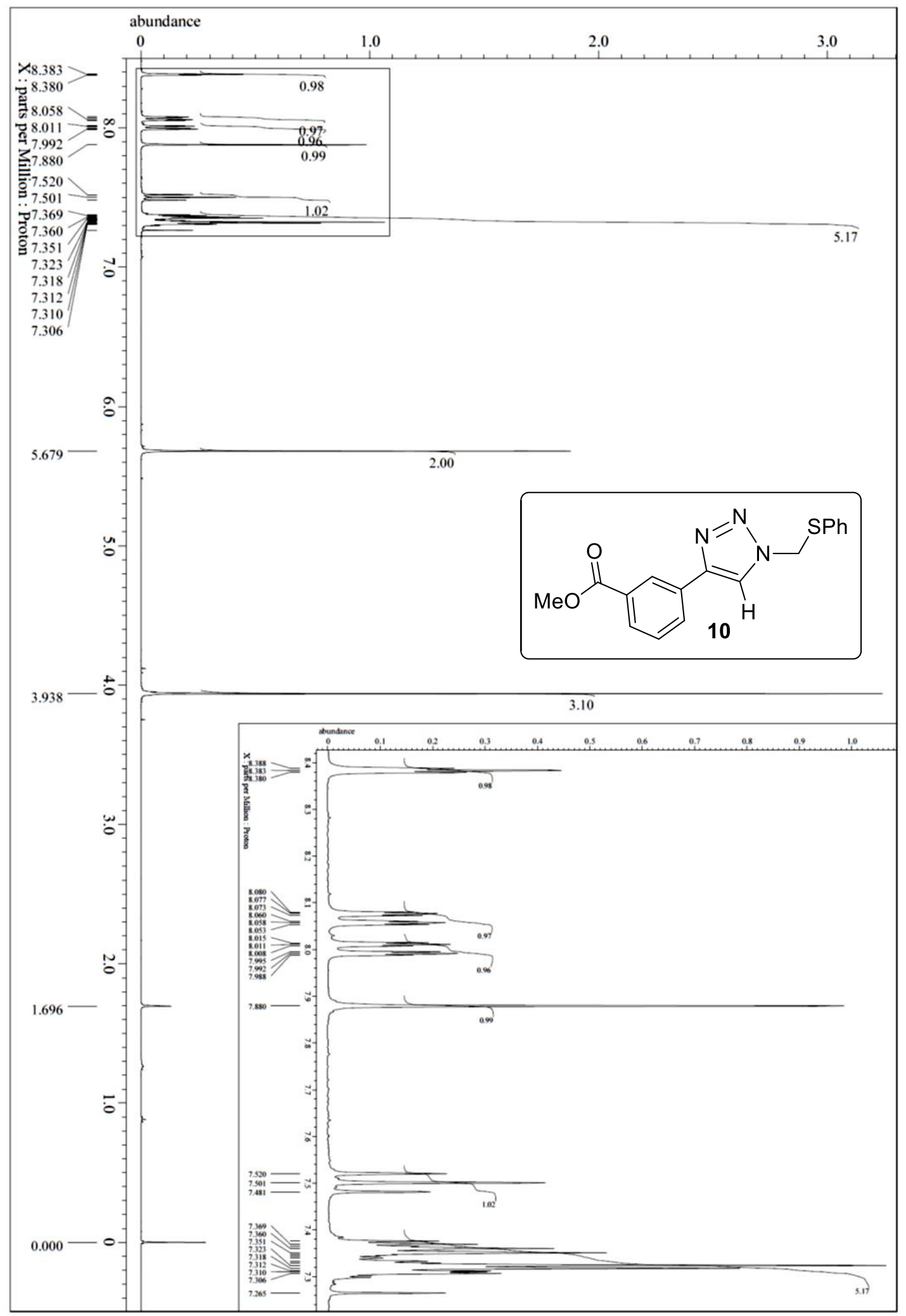




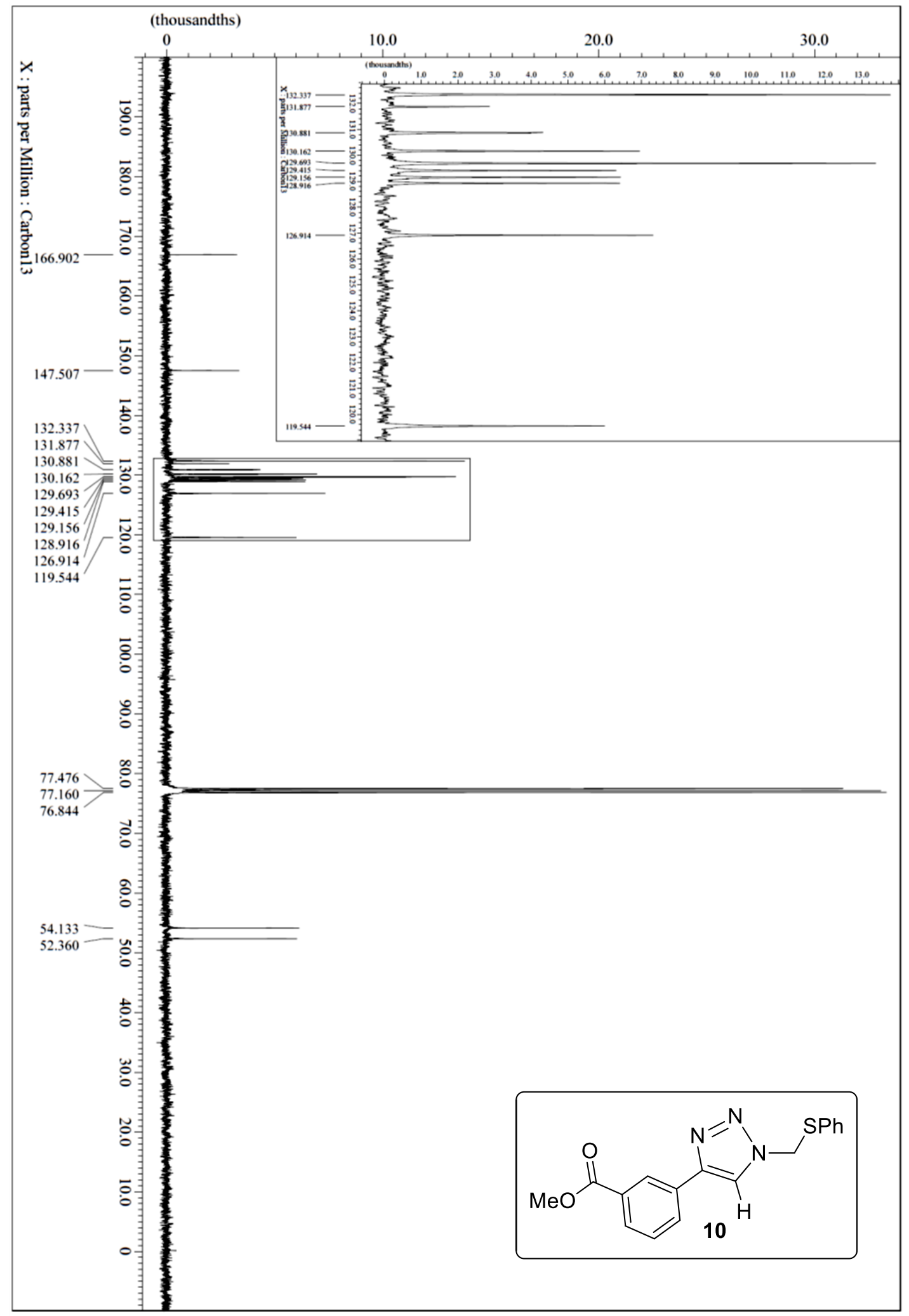

Figure S30. ${ }^{1} \mathrm{H}(400 \mathrm{MHz})$ and ${ }^{13} \mathrm{C}\left\{{ }^{1} \mathrm{H}\right\} \mathrm{NMR}(101 \mathrm{MHz})$ spectra of $\mathbf{1 0}\left(\mathrm{CDCl}_{3}, \mathrm{rt}\right)$ 


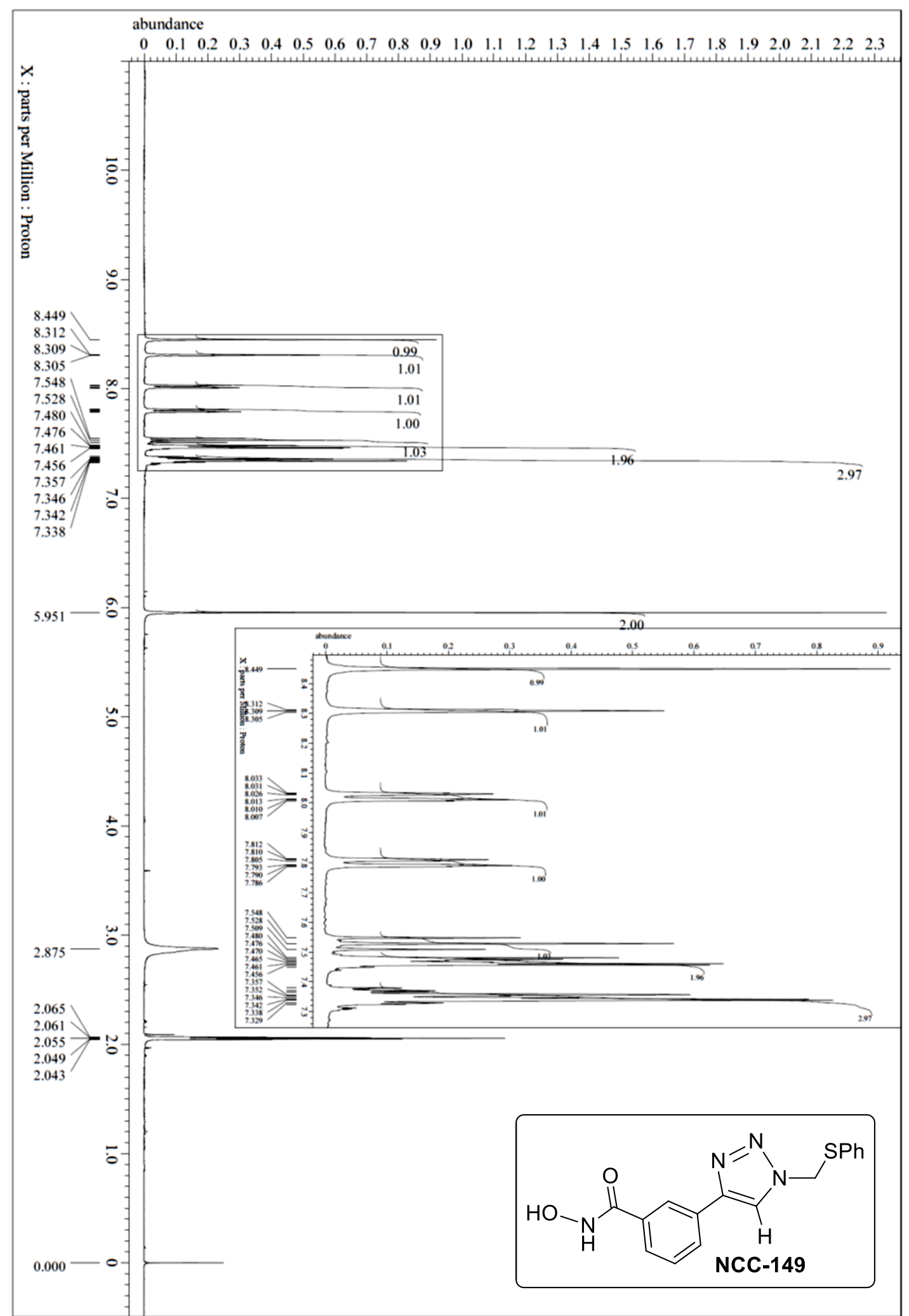




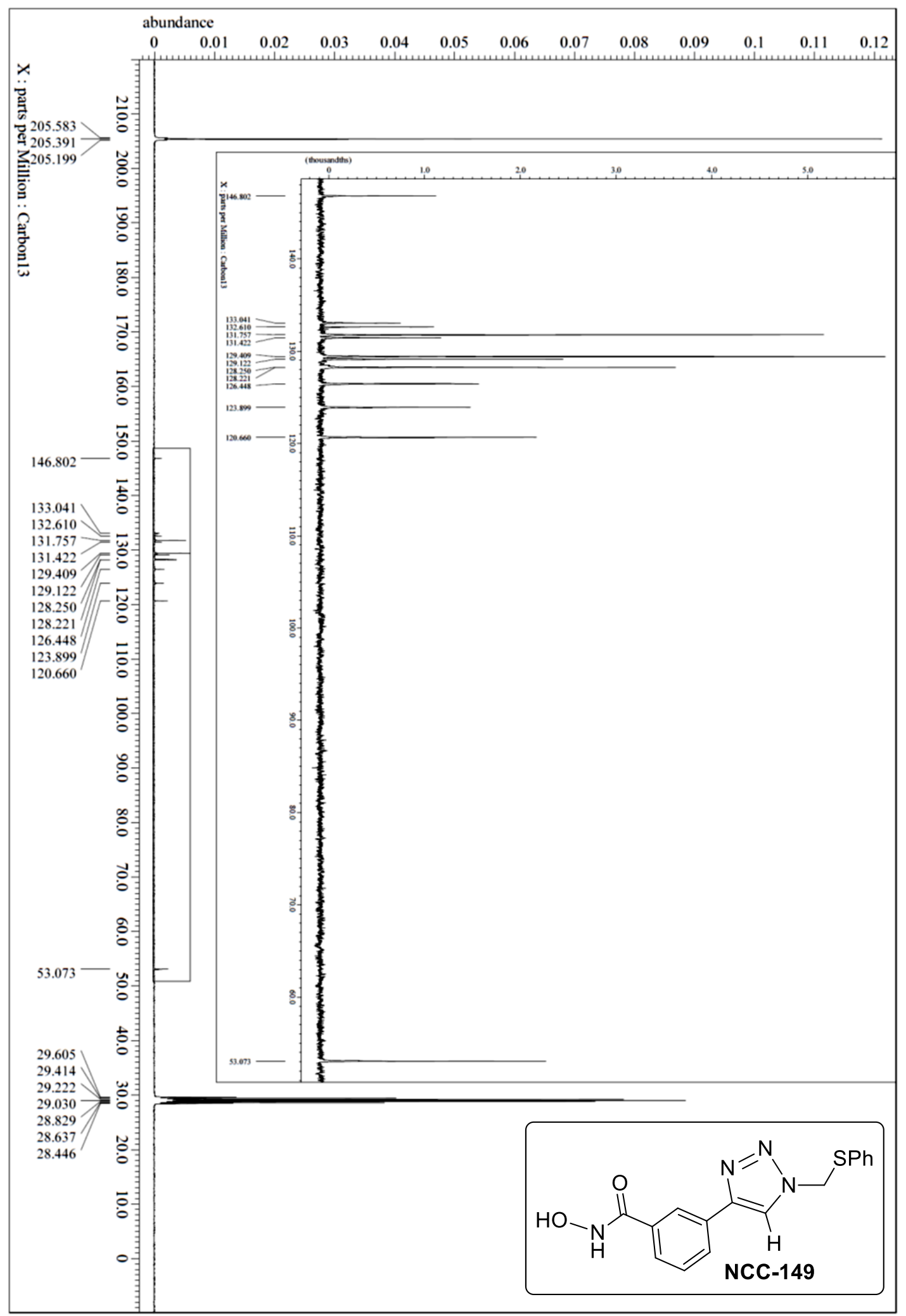

Figure S31. ${ }^{1} \mathrm{H}(400 \mathrm{MHz})$ and ${ }^{13} \mathrm{C}\left\{{ }^{1} \mathrm{H}\right\}$ NMR (101 MHz) spectra of NCC-149 (acetone- $d_{6}$, rt) 